\title{
Xpert MTB/RIF and Xpert Ultra assays for screening for pulmonary tuberculosis and rifampicin resistance in adults, irrespective of signs or symptoms (Review)
}

Shapiro AE, Ross JM, Yao M, Schiller I, Kohli M, Dendukuri N, Steingart KR, Horne DJ

Shapiro AE, Ross JM, Yao M, Schiller I, Kohli M, Dendukuri N, Steingart KR, Horne DJ.

Xpert MTB/RIF and Xpert Ultra assays for screening for pulmonary tuberculosis and rifampicin resistance in adults, irrespective of signs or symptoms.

Cochrane Database of Systematic Reviews 2021, Issue 3. Art. No.: CD013694.

DOI: 10.1002/14651858.CD013694.pub2. 
TABLE OF CONTENTS

HEADER 1

ABSTRACT

PLAIN LANGUAGE SUMMARY

SUMMARY OF FINDINGS

BACKGROUND

Figure 1.

OBJECTIVES

METHODS

RESULTS

Figure 2.

Figure 3.

Figure 4.

Figure 5.

Figure 6.

Figure 7.

Figure 8.

Figure 9.

Figure 10.

DISCUSSION

AUTHORS' CONCLUSIONS

ACKNOWLEDGEMENTS

REFERENCES

CHARACTERISTICS OF STUDIES

DATA

Test 1. Xpert MTB/RIF, HIV positive, irrespective of TB symptoms

Test 2. Xpert Ultra, HIV, irrespective of TB symptoms

Test 3. Xpert MTB/RIF, household contacts, irrespective of TB symptoms

Test 4. Xpert MTB/RIF, prisoners, irrespective of TB symptoms

Test 5. Xpert MTB/RIF, miners, irrespective of TB symptoms

Test 6. Xpert MTB/RIF, admitted patients, irrespective of TB symptoms

Test 7. Xpert MTB/RIF, all high-risk groups

Test 8. Xpert MTB/RIF for rifampicin resistance

ADDITIONAL TABLES

APPENDICES

HISTORY

CONTRIBUTIONS OF AUTHORS

DECLARATIONS OF INTEREST

SOURCES OF SUPPORT

DIFFERENCES BETWEEN PROTOCOL AND REVIEW

INDEX TERMS 


\title{
Xpert MTB/RIF and Xpert Ultra assays for screening for pulmonary tuberculosis and rifampicin resistance in adults, irrespective of signs or symptoms
}

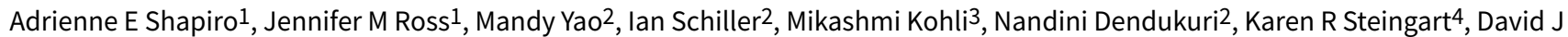 \\ Horne ${ }^{5}$
}

1Division of Allergy \& Infectious Diseases, Global Health \& Medicine, University of Washington, Seattle, USA. ${ }^{2}$ Centre for Outcomes Research, McGill University Health Centre - Research Institute, Montreal, Canada. ${ }^{3}$ Department of Epidemiology, Biostatistics and Occupational Health, McGill University, Montreal, Canada. ${ }^{4}$ Honorary Research Fellow, Department of Clinical Sciences, Liverpool School of Tropical Medicine, Liverpool, UK. 5 Department of Medicine, Division of Pulmonary and Critical Care Medicine, and Firland Northwest TB Center, University of Washington, Seattle, WA, USA

Contact address: Karen R Steingart, karen.steingart@gmail.com.

Editorial group: Cochrane Infectious Diseases Group.

Publication status and date: New, published in Issue 3, 2021.

Citation: Shapiro AE, Ross JM, Yao M, Schiller I, Kohli M, Dendukuri N, Steingart KR, Horne DJ. Xpert MTB/RIF and Xpert Ultra assays for screening for pulmonary tuberculosis and rifampicin resistance in adults, irrespective of signs or symptoms. Cochrane Database of Systematic Reviews 2021, Issue 3. Art. No.: CD013694. DOI: 10.1002/14651858.CD013694.pub2.

Copyright ( 2021 The Authors. Cochrane Database of Systematic Reviews published by John Wiley \& Sons, Ltd. on behalf of The Cochrane Collaboration. This is an open access article under the terms of the Creative Commons Attribution-Non-Commercial Licence, which permits use, distribution and reproduction in any medium, provided the original work is properly cited and is not used for commercial purposes.

\section{A B S T R A C T}

\section{Background}

Tuberculosis is a leading cause of infectious disease-related death and is one of the top 10 causes of death worldwide. The World Health Organization (WHO) recommends the use of specific rapid molecular tests, including Xpert MTB/RIF or Xpert Ultra, as initial diagnostic tests for the detection of tuberculosis and rifampicin resistance in people with signs and symptoms of tuberculosis. However, the WHO estimates that nearly one-third of all active tuberculosis cases go undiagnosed and unreported. We were interested in whether a single test, Xpert MTB/RIF or Xpert Ultra, could be useful as a screening test to close this diagnostic gap and improve tuberculosis case detection.

\section{Objectives}

To estimate the accuracy of Xpert MTB/RIF and Xpert Ultra for screening for pulmonary tuberculosis in adults, irrespective of signs or symptoms of pulmonary tuberculosis in high-risk groups and in the general population. Screening "irrespective of signs or symptoms" refers to screening of people who have not been assessed for the presence of tuberculosis symptoms (e.g. cough).

To estimate the accuracy of Xpert MTB/RIF and Xpert Ultra for detecting rifampicin resistance in adults screened for tuberculosis, irrespective of signs and symptoms of pulmonary tuberculosis in high-risk groups and in the general population.

\section{Search methods}

We searched 12 databases including the Cochrane Infectious Diseases Group Specialized Register, MEDLINE and Embase, on 19 March 2020 without language restrictions. We also reviewed reference lists of included articles and related Cochrane Reviews, and contacted researchers in the field to identify additional studies. 


\section{Selection criteria}

Cross-sectional and cohort studies in which adults (15 years and older) in high-risk groups (e.g. people living with HIV, household contacts of people with tuberculosis) or in the general population were screened for pulmonary tuberculosis using Xpert MTB/RIF or Xpert Ultra. For tuberculosis detection, the reference standard was culture. For rifampicin resistance detection, the reference standards were culturebased drug susceptibility testing and line probe assays.

\section{Data collection and analysis}

Two review authors independently extracted data using a standardized form and assessed risk of bias and applicability using QUADAS-2. We used a bivariate random-effects model to estimate pooled sensitivity and specificity with $95 \%$ credible intervals (Crls) separately for tuberculosis detection and rifampicin resistance detection. We estimated all models using a Bayesian approach. For tuberculosis detection, we first estimated screening accuracy in distinct high-risk groups, including people living with HIV, household contacts, people residing in prisons, and miners, and then in several high-risk groups combined.

\section{Main results}

We included a total of 21 studies: 18 studies (13,114 participants) evaluated Xpert MTB/RIF as a screening test for pulmonary tuberculosis and one study (571 participants) evaluated both Xpert MTB/RIF and Xpert Ultra. Three studies (159 participants) evaluated Xpert MTB/RIF for rifampicin resistance. Fifteen studies (75\%) were conducted in high tuberculosis burden and 16 (80\%) in high TB/HIV-burden countries. We judged most studies to have low risk of bias in all four QUADAS-2 domains and low concern for applicability.

\section{Xpert MTB/RIF and Xpert Ultra as screening tests for pulmonary tuberculosis}

In people living with HIV (12 studies), Xpert MTB/RIF pooled sensitivity and specificity ( $95 \%$ Crl) were $61.8 \%$ (53.6 to 69.9$)$ (602 participants; moderate-certainty evidence) and $98.8 \%$ ( 98.0 to 99.4) (4173 participants; high-certainty evidence). Of 1000 people where 50 have tuberculosis on culture, 40 would be Xpert MTB/RIF-positive; of these, $9(22 \%)$ would not have tuberculosis (false-positives); and 960 would be Xpert MTB/RIF-negative; of these, 19 (2\%) would have tuberculosis (false-negatives).

In people living with HIV (1 study), Xpert Ultra sensitivity and specificity (95\% CI) were 69\% (57 to 80 ) (68 participants; very low-certainty evidence) and 98\% (97 to 99) (503 participants; moderate-certainty evidence). Of 1000 people where 50 have tuberculosis on culture, 53 would be Xpert Ultra-positive; of these, 19 (36\%) would not have tuberculosis (false-positives); and 947 would be Xpert Ultra-negative; of these, $16(2 \%)$ would have tuberculosis (false-negatives).

In non-hospitalized people in high-risk groups (5 studies), Xpert MTB/RIF pooled sensitivity and specificity were $69.4 \%$ (47.7 to 86.2 ) (337 participants, low-certainty evidence) and $98.8 \%$ (97.2 to 99.5) (8619 participants, moderate-certainty evidence). Of 1000 people where 10 have tuberculosis on culture, 19 would be Xpert MTB/RIF-positive; of these, 12 (63\%) would not have tuberculosis (false-positives); and 981 would be Xpert MTB/RIF-negative; of these, 3 (0\%) would have tuberculosis (false-negatives).

We did not identify any studies using Xpert MTB/RIF or Xpert Ultra for screening in the general population.

Xpert MTB/RIF as a screening test for rifampicin resistance

Xpert MTB/RIF sensitivity was $81 \%$ and $100 \%$ (2 studies, 20 participants; very low-certainty evidence), and specificity was $94 \%$ to $100 \%$, (3 studies, 139 participants; moderate-certainty evidence).

\section{Authors' conclusions}

Of the high-risks groups evaluated, Xpert MTB/RIF applied as a screening test was accurate for tuberculosis in high tuberculosis burden settings. Sensitivity and specificity were similar in people living with HIV and non-hospitalized people in high-risk groups. In people living with HIV, Xpert Ultra sensitivity was slightly higher than that of Xpert MTB/RIF and specificity similar. As there was only one study of Xpert Ultra in this analysis, results should be interpreted with caution. There were no studies that evaluated the tests in people with diabetes mellitus and other groups considered at high-risk for tuberculosis, or in the general population.

\section{PLAIN LANGUAGE SUMMARY}

\section{How accurate are sputum Xpert tests for screening for active pulmonary tuberculosis and rifampicin resistance in adults whether or not they have tuberculosis symptoms?}

\section{Why is using Xpert tests to screen for pulmonary tuberculosis important?}

Tuberculosis is the leading cause of infectious disease-related death and one of the top 10 causes of death worldwide. The World Health Organization (WHO) recommends using specific rapid tests as initial tests for diagnosing tuberculosis and rifampicin resistance in people with signs and symptoms of tuberculosis. However, the WHO estimates that nearly one-third of all active tuberculosis cases go undiagnosed and unreported. Not recognizing tuberculosis when it is present (a false negative test result) may result in illness and death and an increased

Xpert MTB/RIF and Xpert Ultra assays for screening for pulmonary tuberculosis and rifampicin resistance in adults, irrespective of signs 
risk of infecting others. An incorrect diagnosis of tuberculosis (false-positive result) may mean that people are given antibiotics when there is no benefit to be gained.

\section{What is the aim of this review?}

To estimate the accuracy of Xpert MTB/RIF and Xpert Ultra as screening tests for pulmonary tuberculosis and rifampicin resistance in adults whether or not they have tuberculosis symptoms (such as cough, fever, weight loss, and night sweats). We were interested in how the tests worked in groups at high risk for tuberculosis, including people living with HIV (PLHIV), household contacts of people with tuberculosis, miners, people residing in prisons, people with diabetes, and in the general public.

\section{What was studied in this review?}

Xpert MTB/RIF and Xpert Ultra are rapid tests for simultaneously diagnosing tuberculosis and rifampicin resistance. We combined study results to determine:

- sensitivity: people with tuberculosis (rifampicin resistance) correctly diagnosed as having the condition.

- specificity: people without tuberculosis (rifampicin resistance) correctly identified as not having the condition.

The closer sensitivity and specificity are to $100 \%$, the better the test.

\section{What are the main results in this review?}

Twenty-one studies: 18 studies (13,114 participants) evaluated Xpert MTB/RIF as a screening test for pulmonary tuberculosis and one study (571 participants) evaluated both Xpert MTB/RIF and Xpert Ultra. Three studies (159 participants) evaluated Xpert MTB/RIF for rifampicin resistance.

For every 1000 people tested, if 50 had tuberculosis according to the reference standard:

PLHIV

- Xpert MTB/RIF (12 studies):

. 40 people would test positive, including 9 without tuberculosis ( $62 \%$ sensitivity)

- 960 people would test negative, including 19 with tuberculosis (99\% specificity)

- Xpert Ultra (1 study):

. 53 people would test positive, including 19 without tuberculosis (69\% sensitivity)

. 947 people would test negative, including 16 with tuberculosis ( $98 \%$ specificity)

For every 1000 people tested, if 10 had tuberculosis according to the reference standard:

Other high-risk groups combined

- Xpert MTB/RIF (5 studies):

- 19 people would test positive, including 12 without tuberculosis (69\% sensitivity)

- 981 people would test negative, including 3 with tuberculosis ( $99 \%$ specificity)

For detection of rifampicin resistance, Xpert MTB/RIF sensitivity was $81 \%$ and $100 \%$ ( 2 studies) and specificity was $94 \%$ to $100 \%$ ( 3 studies).

\section{How reliable are the results of the studies in this review?}

In the included studies, the reference standards for diagnosing pulmonary tuberculosis (culture) and rifampicin resistance (drug susceptibility testing) are likely to have been reliable methods for deciding whether patients really had the conditions. We were fairly confident in the results for Xpert MTB/RIF in PLHIV, and less so for other high-risk groups. Not enough people have been studied to be confident about the results for Xpert Ultra or for detection of rifampicin resistance.

\section{Who do the results of this review apply to?}

Studies were mainly performed in high tuberculosis and high HIV burden settings. No studies evaluated the tests in people with diabetes mellitus or the general population.

\section{What are the implications of this review?}

Xpert MTB/RIF and Xpert Ultra assays for screening for pulmonary tuberculosis and rifampicin resistance in adults, irrespective of signs

Copyright (C) 2021 The Authors. Cochrane Database of Systematic Reviews published by John Wiley \& Sons, Ltd. on behalf of The Cochrane

Collaboration. 
In PLHIV, Xpert MTB/RIF as a screening test was accurate for tuberculosis in high tuberculosis burden settings. In high-risk groups, Xpert MTB/RIF may assist in identifying tuberculosis, but the certainty of evidence is low. In PLHIV, Xpert Ultra sensitivity was slightly higher than that of Xpert MTB/RIF and specificity similar based on one study. There were few studies and few people tested for rifampicin resistance and no studies that evaluated the tests in people with diabetes or in the general population.

\section{How up-to-date is this review?}

19 March 2020. 


\section{SUMMARY OF FINDINGS}

Summary of findings 1. Xpert MTB/RIF as a screening test for pulmonary tuberculosis in people living with HIV and non-hospitalized people in highrisk groups

Review question: what is the accuracy of Xpert MTB/RIF and Xpert Ultra for screening for pulmonary tuberculosis in adults irrespective of signs or symptoms of pulmonary tuberculosis?

Patients/population: people living with HIV and non-hospitalized people in high-risk groups

Setting: community and primary care facilities

Index tests: Xpert MTB/RIF and Xpert Ultra; role: screening test

Threshold for index tests: an automated result is provided

Reference standards: solid or liquid culture

Studies: cross-sectional and cohort studies

\begin{tabular}{|c|c|c|c|c|c|c|c|}
\hline \multirow{2}{*}{$\begin{array}{l}\text { Index test, } \\
\text { population }\end{array}$} & \multirow[t]{2}{*}{ Effect $(95 \%$ CrI) } & \multirow{2}{*}{$\begin{array}{l}\text { Number of } \\
\text { participants } \\
\text { (studies) }\end{array}$} & \multirow[t]{2}{*}{ Test result } & \multicolumn{3}{|c|}{ Number of results per 1000 patients tested $(95 \% \mathrm{Cl})$} & \multirow{2}{*}{$\begin{array}{l}\text { Certainty of } \\
\text { the evidence } \\
\text { (GRADE) }\end{array}$} \\
\hline & & & & Prevalence $0.5 \%$ & Prevalence $5 \%$ & Prevalence $10 \%$ & \\
\hline \multirow{4}{*}{$\begin{array}{l}\text { Xpert MTB/ } \\
\text { RIF, people } \\
\text { living with HIV }\end{array}$} & Pooled sensitivity & \multirow[t]{2}{*}{$602(12)$} & True positives & $3(3$ to 3$)$ & 31 (27 to 35$)$ & 62 (54 to 70$)$ & \multirow{2}{*}{$\begin{array}{l}\oplus \oplus \bigcirc \\
\text { Moderatea,b }\end{array}$} \\
\hline & $61.8 \%(53.6$ to 69.9$)$ & & False negatives & $2(2$ to 2$)$ & 19 (15 to 23$)$ & 38 (30 to 46$)$ & \\
\hline & Pooled specificity & \multirow[t]{2}{*}{$4173(12)$} & True negatives & 985 (975 to 985$)$ & 941 (931 to 941$)$ & 891 (882 to 891$)$ & \multirow{2}{*}{$\begin{array}{l}\text { High } \\
\text { Hign }\end{array}$} \\
\hline & $98.8 \%(98.0$ to 99.4$)$ & & False positives & $10(10$ to 20$)$ & 9 (9 to 19$)$ & 9 (9 to 18$)$ & \\
\hline \multirow{5}{*}{$\begin{array}{l}\text { Xpert Ultra, } \\
\text { people living } \\
\text { with HIV }\end{array}$} & Sensitivity & \multirow[t]{2}{*}{$68(1)$} & True positives & $3(3$ to 4$)$ & 34 (28 to 40$)$ & 69 (57 to 80$)$ & \multirow{2}{*}{$\begin{array}{l}\oplus 000^{c, d} \\
\text { Very low }\end{array}$} \\
\hline & $69 \%$ (57 to 80$)$ & & False negatives & $2(1$ to 2$)$ & 16 (10 to 22$)$ & 31 (20 to 43$)$ & \\
\hline & Specificity & \multirow[t]{2}{*}{$503(1)$} & True negatives & 975 (965 to 985$)$ & 931 (922 to 941$)$ & $882(873$ to 891$)$ & \multirow{2}{*}{$\begin{array}{l}\oplus \oplus \oplus \circ \subset \\
\text { Moderate }\end{array}$} \\
\hline & \multirow[t]{2}{*}{$98 \%$ (97 to 99 ) } & & False positives & 20 (10 to 30$)$ & 19 (9 to 28$)$ & 18 (9 to 27 ) & \\
\hline & & & & Prevalence $0.5 \%$ & Prevalence $1 \%$ & Prevalence $2 \%$ & $\begin{array}{l}\text { Certainty of } \\
\text { the evidence } \\
\text { (GRADE) }\end{array}$ \\
\hline \multirow{2}{*}{$\begin{array}{l}\text { Xpert MTB/ } \\
\text { RIF, non-hos- } \\
\text { pitalized peo- } \\
\text { ple in high- } \\
\text { risk groups }\end{array}$} & \multirow{2}{*}{$\begin{array}{l}\text { Pooled sensitivity } 69.4 \% \\
\text { ( } 47.7 \text { to } 86.2 \text { ) }\end{array}$} & \multirow[t]{2}{*}{$337(5)$} & True positives & $3(2$ to 4$)$ & 7 (5 to 9$)$ & 14 (10 to 17$)$ & \multirow{2}{*}{$\begin{array}{l}\oplus \oplus 0 \mathrm{e}^{\mathrm{f}, \mathrm{g}} \\
\text { Low }\end{array}$} \\
\hline & & & False negatives & $2(1$ to 3$)$ & $3(1$ to 5$)$ & $6(3$ to 10$)$ & \\
\hline
\end{tabular}


Studies: cross-sectional and cohort studies

\begin{tabular}{|c|c|c|c|c|c|c|c|}
\hline \multirow[t]{2}{*}{ Index test } & \multirow[t]{2}{*}{ Effect } & \multirow{2}{*}{$\begin{array}{l}\text { Number of } \\
\text { participants } \\
\text { (studies) }\end{array}$} & \multirow[t]{2}{*}{ Test result } & \multicolumn{3}{|c|}{ Number of results per 1000 patients tested } & \multirow{2}{*}{$\begin{array}{l}\text { Certainty of the evi- } \\
\text { dence (GRADE) }\end{array}$} \\
\hline & & & & Prevalence $0.5 \%$ & Prevalence $1 \%$ & Prevalence $2 \%$ & \\
\hline \multirow[t]{4}{*}{ Xpert MTB/RIF } & Sensitivity $81 \%$ & $20(2)$ & True positives & 4 to 5 & 8 to 10 & 16 to 20 & $\oplus \circ \bigcirc^{a, b, c}$ \\
\hline & & & False negatives & 0 to 1 & 0 to 2 & 0 to 4 & Very low \\
\hline & Specificity $94 \%$ & $139(3)$ & True negatives & 935 to 995 & 931 to 990 & 921 to 980 & $\oplus \oplus \oplus \mathrm{O}^{\mathrm{d}, \mathrm{e}}$ \\
\hline & & & False positives & 0 to 60 & 0 to 59 & 0 to 59 & Moderate \\
\hline
\end{tabular}

Prevalence estimates were suggested by the WHO Global TB Programme. The prevalence of rifampicin resistance in the studies was $7.3 \%$ and $16.7 \%$.

\section{Explanations}

aThere were only two studies included in this analysis, conducted in sub-Saharan Africa. The prevalence of rifampicin resistance in the studies was higher than those presented in the table. The applicability to other settings comes with some uncertainty. We downgraded one level for indirectness.

bThere was a wide range of sensitivities of Xpert MTB/RIF for detection of rifampicin resistance between the two included studies: $81 \%$ and $100 \%$. We downgraded one level for inconsistency.

CThere were few participants contributing to this analysis. We already downgraded one level for inconsistency. We downgraded one level for imprecision.

dof the three included studies, two were conducted in southern Africa, one in Malaysia. Applicability to other settings comes with some uncertainty. We downgraded one level for indirectness.

eThe specificities were $94 \%, 97 \%$, and $100 \%$. One explanation for the lower specificity of $94 \%$ is a problem identified with the Xpert MTB/RIF assay, which was modified to improve specificity after publication of this study. We did not downgrade for imprecision.

\section{GRADE certainty of the evidence.}

High: we are very confident that the true effect lies close to that of the estimate of the effect.

Moderate: we are moderately confident in the effect estimate: the true effect is likely to be close to the estimate of the effect, but there is a possibility that it is substantially different.

Low: our confidence in the effect estimate is limited: the true effect may be substantially different from the estimate of the effect.

Very low: we have very little confidence in the effect estimate: the true effect is likely to be substantially different from the estimate of effect.

The results presented in this table should not be interpreted in isolation from the results of individual included studies contributing to each summary test accuracy measure. 


\section{B A C K G R O U N D}

Tuberculosis is the world's leading cause of infectious diseaserelated death and is one of the top 10 causes of death worldwide. In 2019, an estimated 10 million people developed tuberculosis disease (WHO Global TB Report 2020).

Among all tuberculosis cases, about $8 \%$ were in people living with HIV (WHO Global TB Report 2020). The risk of developing tuberculosis is much higher in people living with HIV, estimated to be 20 to 37 times higher in HIV-positive individuals than in HIV-negative individuals (Getahun 2010). Signs and symptoms of tuberculosis in people living with HIV vary, which makes it challenging to determine when to consider a diagnosis of tuberculosis - tuberculosis is the leading cause of hospitalisation and death in people with HIV worldwide (Ford 2016). In addition, there were around 500,000 new cases of rifampicin-resistant tuberculosis, of which $78 \%$ had multidrug-resistant tuberculosis (tuberculosis that is resistant to both rifampicin and isoniazid, the two most essential anti-tuberculosis drugs) (WHO Global TB Report 2020). When tuberculosis is detected early and is effectively treated the disease is largely curable. Ending the tuberculosis epidemic by 2030 is among the health-related targets described in United Nations Sustainable Development Goal 3 (WHO END TB 2015). The United Nations Sustainable Development Goals represent a collective plan to end poverty, decrease inequality, and protect the planet from degradation by 2030 (UN Sustainable Development Goals 2030).

The World Health Organization (WHO) recommends the use of specific rapid molecular tests, including Xpert MTB/RIF or Xpert Ultra, the newest version of the assay, as the initial diagnostic tests for the detection of tuberculosis and rifampicin resistance in people with signs and symptoms of tuberculosis (WHO Consolidated Guidelines (Module 3) 2020). However, the WHO estimates that nearly one-third of all active tuberculosis cases go undiagnosed and unreported (WHO Global TB Report 2020). In an effort to close this diagnostic gap, the WHO is seeking evidence to recommend case-finding approaches and strategies to improve tuberculosis case detection of the 'missing millions'. In particular, the WHO is interested in case-finding approaches in high-risk groups and settings, such as people living with HIV, people with diabetes mellitus, and people residing in prisons. Stated another way, the WHO is interested in the best ways to find the so-called 'missing millions'.

Tuberculosis screening is a term that has been used differently in the literature depending on the context. We use tuberculosis screening as defined by the WHO: the "systematic identification of people with suspected active TB [tuberculosis], in a predetermined target group, using tests, examinations or other procedures that can be applied rapidly." Further, we define intensified case-finding as tuberculosis screening activities set in health facilities, and active case-finding as tuberculosis screening activities set in the community, including household-based or residence-based screening activities (WHO Systematic screening 2013). The EndTB strategy emphasizes early diagnosis of tuberculosis, including universal drug susceptibility testing, and systematic screening of contacts and high-risk groups (WHO Global TB Report 2020).

Current screening approaches for active tuberculosis typically recommend initial screening of people living with HIV for four cardinal signs and symptoms of tuberculosis: cough, fever, weight loss, and night sweats, or people who do not have HIV, the single symptom of prolonged cough. People with a positive symptom screen then may go on to receive additional screening with a chest X-ray and diagnostic testing using sputum Xpert MTB/RIF or Xpert Ultra as recommended. Concerning people living with HIV, a recent systematic review found that the four-symptom screen had lower sensitivity and specificity for active tuberculosis in HIVpositive people on antiretroviral therapy (ART) than in HIV-positive people not taking ART (Hamada 2018). Compared to Xpert MTB/ RIF, Xpert Ultra has shown increased sensitivity for tuberculosis in HIV-positive people (Dorman 2018). WHO Tuberculosis Standard 8 states, "For persons living with HIV, the Xpert MTB/RIF Ultra assay should be used as an initial diagnostic test" (WHO Compendium of WHO guidelines 2018). Recent population surveys using chest radiography, irrespective of symptoms, as the initial screen for tuberculosis (followed by diagnostic testing) have identified a substantial burden of subclinical tuberculosis in people with and without HIV, supporting a need for new approaches to screen and identify active tuberculosis using more sensitive tools (Frascella 2020; Gunasekera 2020).

Several Cochrane Reviews have been published or are in process to assess the diagnostic accuracy of Xpert MTB/RIF and Xpert Ultra for different target conditions and in various populations. Of relevance to the current review, recent Cochrane Reviews found Xpert MTB/ RIF and Xpert Ultra to be highly sensitive and specific for pulmonary tuberculosis and rifampicin resistance in adults with signs and symptoms of tuberculosis; see Index test(s) (Horne 2019; Zifodya 2021). The current review determined the accuracy of Xpert MTB/ RIF and Xpert Ultra for tuberculosis and rifampicin resistance in adults, irrespective of signs and symptoms of tuberculosis, that is, when used as a screening test. Screening "irrespective of signs or symptoms" refers to screening of people who have not been assessed for the presence of tuberculosis symptoms (e.g. cough). This can include both asymptomatic (people without symptoms of tuberculosis) and people with symptoms of tuberculosis.

\section{Target condition being diagnosed}

Tuberculosis is caused by the bacterium Mycobacterium tuberculosis (M tuberculosis) and is spread from person to person through the air. Tuberculosis most commonly affects the lungs (pulmonary tuberculosis), but may affect any organ or tissue outside of the lungs (extrapulmonary tuberculosis). Signs and symptoms of pulmonary tuberculosis include cough, fever, chills, night sweats, weight loss, haemoptysis (coughing up blood), and fatigue. Signs and symptoms of extrapulmonary tuberculosis depend on the site of disease. Tuberculosis treatment regimens must contain multiple drugs, to which the organisms are sensitive, to cure tuberculosis and avoid selection for drug resistance. In 2019, there were approximately half a million new cases of rifampicin-resistant tuberculosis, of which $78 \%$ were multidrugresistant (MDR-TB) (WHO Global TB Report 2020), The treatment of MDR-TB is complex, historically requiring two years or more of therapy, although the WHO conditionally recommended a regimen of nine to 12 months in 2016 (WHO Guidelines 2016). The drugs used to treat MDR-TB are less potent and more toxic than the drugs used to treat drug-susceptible tuberculosis. WHO guidance states that "All patients with MDR-TB or rifampicinresistant tuberculosis, including those with additional resistance to fluoroquinolones, stand to benefit from effective all-oral treatment regimens, either shorter or longer, implemented under

Xpert MTB/RIF and Xpert Ultra assays for screening for pulmonary tuberculosis and rifampicin resistance in adults, irrespective of signs 
programmatic conditions" (WHO Consolidated Guidelines (Module 4) 2020).

\section{Index test(s)}

Xpert MTB/RIF is an automated polymerase chain reaction (PCR) test (molecular test) using the GeneXpert platform (Cepheid 2019). Xpert MTB/RIF is a single test that can detect both $M$ tuberculosis complex and rifampicin resistance within two hours after starting the test, with minimal hands-on technical time. Unlike conventional nucleic acid amplification tests, (NAATs), Xpert MTB/RIF integrates sample processing and PCR amplification and detection into a single cartridge. Following sample loading, all steps in the assay are completely automated and self-contained. In addition, the assay's sample reagent, used to liquefy sputum, has potent tuberculocidal (the ability to kill tuberculosis bacteria) properties and so largely eliminates biosafety concerns during the test procedure (Banada 2010). Xpert MTB/RIF requires an uninterrupted and stable electrical power supply, temperature control, and yearly calibration of the cartridge modules (Global Laboratory Initiative 2019).

Since Xpert MTB/RIF was released, there have been four generations of the test (G1, G2, G3, and G4), involving different software and cartridge combinations. G4 contains modifications that improved determination of rifampicin resistance detection as previous Xpert MTB/RIF versions had found that some rifampicin susceptibility results were falsely resistant. Our previous review identified considerable overlap of the accuracy estimates for Xpert MTB/RIF across generations of the test, suggesting that the difference in test generations was unlikely to contribute meaningfully to heterogeneity in accuracy estimates (Steingart 2014). In order to improve on Xpert MTB/RIF sensitivity, Cepheid developed Xpert MTB/RIF Ultra (hereafter referred to as Xpert Ultra), a re-engineered assay that uses a newly developed cartridge but may be run on the same device after a software upgrade. Xpert Ultra incorporates two different multi-copy amplification targets and a larger DNA reaction chamber than Xpert MTB/RIF (WHO Xpert Ultra 2017). A laboratory study reported that the limit of detection using Xpert Ultra improved to $15.6 \mathrm{CFU} / \mathrm{mL}$ of sputum compared to 112.6 CFU/mL for Xpert MTB/RIF (Chakravorty 2017). Of note, Xpert Ultra has added a new result category, 'trace call', that corresponds to the lowest bacillary burden for $M$ tuberculosis detection (WHO Xpert Ultra 2017). Although no result for rifampicin resistance will be available for people with trace results, a trace-positive result is sufficient to initiate anti-tuberculosis therapy in children or HIV-positive people, according to the WHO report. Xpert Ultra is available for clinical use and several countries have moved from using Xpert MTB/RIF to using Xpert Ultra instead. In this Cochrane Review, we included studies that used any generation of the index tests.

Regarding the accuracy of Xpert MTB/RIF and Xpert Ultra for diagnosis of pulmonary tuberculosis in people with signs and symptoms, a recent Cochrane Review found pooled sensitivity and specificity (95\% credible interval) against culture were 90.9\% (86.2 to 94.7 ) and $95.6 \%$ (93.0 to 97.4) for Xpert Ultra (7 studies, 2834 participants; high-certainty evidence) and $84.7 \%$ (78.6 to 89.9 ) and 98.4\% (97.0 to 99.3) for Xpert MTB/RIF (7 studies, 2835 participants; high-certainty evidence), For detection of rifampicin resistance, pooled sensitivity and specificity were $94.9 \%$ (88.9 to 97.9$)$ and 99.1\% (97.7 to 99.8) for Xpert Ultra (5 studies, 921 participants; highcertainty evidence) versus $95.3 \%$ (90.0 to 98.1 ) and $98.8 \%$ (97.2 to 99.6) for Xpert MTB/RIF ( 5 studies, 930 participants; high-certainty evidence) (Zifodya 2021).

\section{Clinical pathway}

There are two complementary approaches to detection of active tuberculosis, Figure 1. The first is the patient-initiated pathway, also known as passive case finding. The second is the provider-initiated screening pathway, which represents the analytic framework for this review (WHO Systematic screening 2015). The index test, either Xpert MTB/RIF or Xpert Ultra, would be performed as the only test for pulmonary tuberculosis and rifampicin resistance in adults, irrespective of signs or symptoms of pulmonary tuberculosis, in high-risk groups and in primary health facilities or community settings. 
Figure 1. There are two complementary approaches to detection of active tuberculosis. The first is the patientinitiated pathway, also known as passive case finding. The second is the provider-initiated screening pathway (WHO Systematic screening 2015), which represents the analytic framework for this review. In the latter pathway, the index test would be applied as the only test, to adults, irrespective of signs and symptoms of tuberculosis, in highrisk groups and in primary health facilities or community settings.

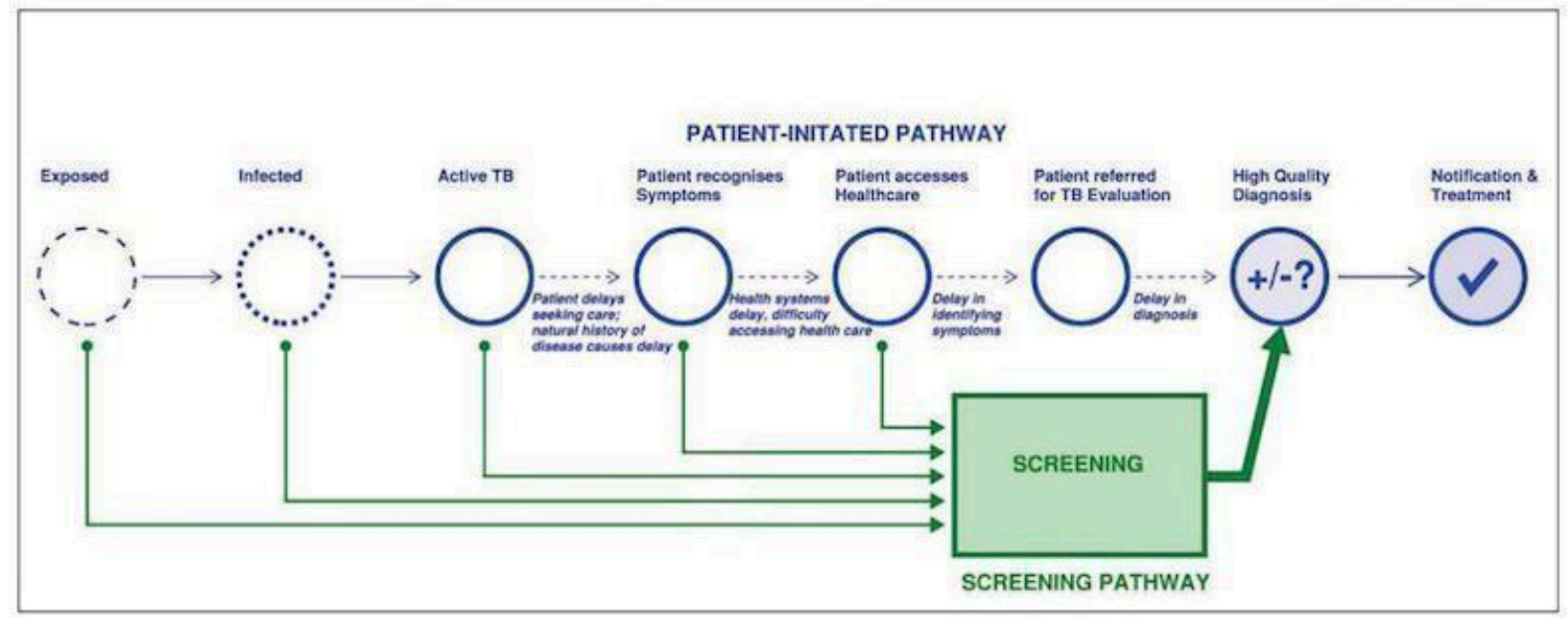

The purpose of the index tests is screening.

The role of the index tests is replacement for usual practice. This may include replacement for the WHO four-question symptom screen.

The downstream consequences of screening include the following

- True-positive (TP): patients would benefit from rapid diagnosis and initiation of appropriate treatment.

- True-negative (TN): patients would be spared unnecessary treatment and would benefit from reassurance, pursuit of an alternative diagnosis if they have symptoms, and determination of eligibility for tuberculosis preventive therapy if indicated.

- False-positive (FP): patients would probably experience anxiety and morbidity caused by additional testing, unnecessary treatment, and possible adverse events; possible stigma associated with a tuberculosis or MDR-TB diagnosis; and the chance that a false-positive result may halt further diagnostic evaluation of the true underlying condition.

- False-negative (FN): patients would experience an increased risk of morbidity and mortality, and delayed or inappropriate treatment initiation; there would be risk of ongoing tuberculosis transmission.

\section{Alternative test(s)}

Alternative screening tests for tuberculosis include no screening (or passive case-finding), and one or more of symptom screening (such as the WHO four-question symptom screen) and chest X-ray, which must be further confirmed with a diagnostic test. Other tools that may be useful in screening include urine lipoarabinomannan (LAM) testing and smear microscopy, which require additional definitive drug resistance testing even if used as simultaneous screening and diagnostic tests. We have previously described selected alternative tests for detection of pulmonary tuberculosis and rifampicin resistance (Horne 2019; Lewinsohn 2017; Unitaid
2017). A Special Collection curated by Cochrane contributors includes Cochrane Reviews from Cochrane Infectious Diseases and other systematic reviews from other international teams. The Special Collection describes key WHO guidelines on tuberculosis diagnostics, and their underpinning systematic reviews (Cochrane Special Collection 2020). Below we review screening tools and highlight several recent developments in tuberculosis diagnostics.

Numerous symptoms, singly and in combination, have been proposed to screen for tuberculosis in different settings. A healthcare or community worker asks the person being screened if they are experiencing any of the selected symptoms, and those who report symptoms according to local criteria go on to receive additional testing such as chest X-ray or diagnostic testing. The most commonly assessed symptoms are cough (varying duration), fever, weight loss, drenching night sweats, loss of appetite, haemoptysis, and fatigue. Single symptoms have modest to low sensitivity; defining a positive screen as any one or more of multiple symptoms improves sensitivity but reduces specificity, consequently increasing the number of diagnostic confirmatory tests. Accuracy of symptom screening varies with the HIV status of the people screened. One study found that any one of cough of any duration, fever of any duration, or night sweats lasting three or more weeks was the most sensitive combination of symptoms for identification of tuberculosis in people living with HIV (93\% sensitivity, 36\% specificity; Cain 2010). In mixed HIV-positive and HIV-negative populations, a single symptom of cough of greater than two weeks' duration identified 35\% (95\% confidence interval (CI) 24 to 46 ) of adults with culture-positive pulmonary tuberculosis in one systematic review and modelling analysis; any one of a list of tuberculosis symptoms had $70 \%$ sensitivity and $61 \%$ specificity for pulmonary tuberculosis in low-HIV-prevalence settings (van't Hoog 2013).

Chest X-ray can involve posterior-anterior, anterior-posterior, or lateral recording, or a combination of two or all of these. Major types of chest X-ray include conventional chest X-ray (producing 36

Xpert MTB/RIF and Xpert Ultra assays for screening for pulmonary tuberculosis and rifampicin resistance in adults, irrespective of signs 10 or symptoms (Review)

Copyright (C) 2021 The Authors. Cochrane Database of Systematic Reviews published by John Wiley \& Sons, Ltd. on behalf of The Cochrane Collaboration. 
$\mathrm{cm} \times 43 \mathrm{~cm}$ film), digital radiography, and computed radiography. Chest X-ray findings including hilar lymphadenopathy, cavitary lesions, and evidence of granulomas can all suggest pulmonary tuberculosis, but are also nonspecific and must be confirmed with additional testing. Accurate interpretation of pulmonary tuberculosis findings on chest $\mathrm{X}$-ray are dependent on the ability of the individual interpreting the chest X-ray, and wide interobserver variation has been reported (Zellweger 2006). Computeraided interpretation of chest X-ray for pulmonary tuberculosis is a promising new technology, especially for resource-limited settings where expertise in chest X-ray interpretation is limited (Harris 2019).

Smear microscopy is the examination of smears for acidfast bacilli (tuberculosis bacteria) under a microscope. The examination may be performed by light microscopy (ZiehlNeelsen), fluorescence microscopy, or light-emitting diode (LED) fluorescence microscopy. Microscopy cannot distinguish between drug-susceptible tuberculosis and drug-resistant tuberculosis. The WHO recommends that microscopy, as the initial diagnostic test, should be replaced with WHO-recommended rapid tests that can simultaneously detect tuberculosis and tuberculosis drug resistance (WHO Consolidated Guidelines (Module 3) 2020).

Nucleic acid amplification tests (NAATs) are molecular systems that can detect small quantities of genetic material (DNA or RNA) from micro-organisms, such as $M$ tuberculosis. The key advantage of NAATs is that they are rapid diagnostic tests, potentially providing results in a few hours. Several new commercial NAATs are in the diagnostic pipeline or have recently come to market (e.g. Truenat MTB, Truenat MTBplus, and Truenat MTB-RIF Dx, Molbio Diagnostics, India). Truenat MTB and Truenat MTB Plus assays show comparable accuracy with Xpert MTB/RIF and Xpert Ultra for detection of tuberculosis, and for sequential detection of rifampicin resistance (Truenat MTB-Rif Dx) (WHO Consolidated Guidelines (Module 3) 2020).

Alere Determine TB LAM Ag (AlereLAM, Alere Inc, Waltham, USA) is a commercially available, point-of-care test for tuberculosis disease (pulmonary and extrapulmonary tuberculosis). The test detects lipoarabinomannan (LAM), a component of the bacterial cell wall, which is present in the urine of some people with tuberculosis. AlereLAM is performed by placing urine on one end of a test strip, with results appearing as a band on the strip if tuberculosis is present. The test is simple, requires no special equipment, and shows results in 25 minutes (Bjerrum 2019). In two randomized trials, the use of Alere LAM in HIV-positive inpatients has been shown to reduce mortality (Gupta-Wright 2018; Peter 2016). Based on evidence from the randomized trials and a Cochrane Review (Bjerrum 2019), the WHO recommends that AlereLAM should be used to assist in the diagnosis of active tuberculosis in HIV-positive adults, adolescents and children. The full recommendations, which differ for inpatients and outpatients, are described here: (WHO Consolidated Guidelines (Module 3) 2020).

Fujifilm SILVAMP TB LAM (FujiLAM, co-developed by Foundation for Innovative New Diagnostics (FIND), Geneva, Switzerland and Fujifilm, Tokyo, Japan) is a new, urine-based, point-of-care test for tuberculosis diagnosis in people living with HIV. In an individual participant data meta-analysis that included five cohorts of people living with HIV, FujiLAM was found to have superior sensitivity, $70.7 \%$ (95\% CI 59.0\% to $80.8 \%$ ), compared to AlereLAM sensitivity of $42.3 \%$ (31.7\% to $51.8 \%$ ), against a microbiological reference standard; FujiLAM had lower specificity, 90.9\% (87.2\% to $93.7 \%)$, compared to AlereLAM specificity of 95.3\% (92.2\% to 97.7\%) (Broger 2020).

Alternative molecular methods for drug susceptibility testing include the commercial line probe assays, GenoType MTBDRplus assay (MTBDRplus, Hain LifeScience, Nehren, Germany), and the Nipro NTM+MDRTB detection kit 2 (Nipro, Tokyo, Japan), which detect the presence of mutations associated with drug resistance to isoniazid and rifampicin (WHO Consolidated Guidelines (Module 3) 2020). Advantages of line probe assays are that they can provide a result for detection of tuberculosis and drug resistance in one to two days. Drawbacks are that line probe assays are expensive and need to be used in intermediate and central laboratories (Unitaid 2017).

\section{Rationale}

Since 2010, the WHO has recommended the use of Xpert MTB/ RIF as the preferred initial diagnostic test for people thought to have MDR-TB or HIV-associated tuberculosis (WHO 2011). In 2013, the WHO expanded the recommendations, stating that Xpert MTB/RIF may be used rather than conventional microscopy and culture as the initial diagnostic test in all adults suspected of having tuberculosis (conditional recommendation acknowledging resource implications, high-quality evidence; WHO Xpert MTB/RIF 2013). In addition, the WHO recommended that following an Xpert MTB/RIF test that demonstrates rifampicin resistance, subsequent drug susceptibility testing (e.g. using a line probe assay for secondline drugs) remains essential to detect resistance to drugs other than rifampicin (WHO Xpert MTB/RIF 2013). In 2017, based on a non-inferiority analysis of Xpert Ultra compared with Xpert MTB/RIF (Dorman 2018), the WHO stated that recommendations on the use of Xpert MTB/RIF also apply to the use of Xpert Ultra as the initial diagnostic test for all adults and children with signs and symptoms of tuberculosis (WHO Consolidated Guidelines (Module 3) 2020).

Given the demonstrated success of rapid molecular tests for diagnosing tuberculosis, we were interested whether a single test, Xpert MTB/RIF or Xpert Ultra, can be useful as a screening test to identify people with active pulmonary tuberculosis in high-risk groups and the in the general population. The settings were community settings or healthcare settings attended for reasons unrelated to tuberculosis. This is a different approach than diagnosing active tuberculosis in people with signs and symptoms of tuberculosis who seek care in health facilities. We performed this Cochrane Review to inform an updated WHO policy on tuberculosis screening, 2020 Revision of the Guidelines for Systematic Screening for Active Tuberculosis: Updated and Consolidated Recommendations and Implementation Guidance (WHO Rapid Communication 2020). The 2020 WHO guidelines also include Cochrane and non-Cochrane systematic reviews on symptom screening, chest radiography, and other tests and strategies for screening for tuberculosis in adults and children.

\section{O B J E C T IVES}

To estimate the accuracy of Xpert MTB/RIF and Xpert Ultra for screening of pulmonary tuberculosis in adults in the following highrisk groups.

- People living with HIV.

- Household contacts of people with tuberculosis.

- People residing in prisons.

Xpert MTB/RIF and Xpert Ultra assays for screening for pulmonary tuberculosis and rifampicin resistance in adults, irrespective of signs 
- Miners.

- Patients residing in high tuberculosis burden settings attending primary health facilities.

- People experiencing homelessness.

- People with diabetes mellitus.

- People who abuse alcohol.

- People who smoke.

- Healthcare workers.

To estimate the accuracy of Xpert MTB/RIF and Xpert Ultra for screening for tuberculosis in adults, irrespective of signs or symptoms of pulmonary tuberculosis in the general population (i.e. low-risk population).

To estimate the accuracy of Xpert MTB/RIF and Xpert Ultra for the detection of rifampicin resistance in the high-risk groups and settings described above and in the general population.

\section{Secondary objectives}

To compare the accuracy of Xpert MTB/RIF and Xpert Ultra in the above high-risk groups and settings and in the general population.

To investigate potential sources of heterogeneity in accuracy estimates, including the percentage of participants with tuberculosis symptoms, tuberculosis burden, and tuberculosis/HIV burden (tuberculosis detection), and MDR-TB burden (rifampicin resistance detection).

\section{METHODS}

\section{Criteria for considering studies for this review}

\section{Types of studies}

We included cross-sectional studies and cohort studies that estimated the accuracy of one or both index tests for both pulmonary tuberculosis and rifampicin resistance or pulmonary tuberculosis alone. We used abstracts to identify published studies and included the full publications when they met our inclusion criteria. We only included studies that reported data comparing the index test(s) to an acceptable reference standard from which we could extract true positive (TP), true negative (TN), false positive (FP), and false negative (FN) values. The index tests could be assessed alone or together with other tests. We included studies designed to find people with active tuberculosis in community settings. We included abstracts with sufficient data to populate a $2 \times 2$ contingency table.

We excluded case reports and studies with a case-control design, the latter because these types of studies are prone to bias, in particular, studies enrolling participants with severe disease and healthy participants without disease. We excluded drug resistance surveys.

\section{Participants}

Adults, defined as 15 years of age and older, irrespective of signs or symptoms of pulmonary tuberculosis in high-risk groups and in the general population. High-risk groups included the following:

- People living with HIV.

- Household contacts of people with tuberculosis.

- People residing in prisons.
- Miners.

- Patients attending primary health facilities.

- Homeless people.

- People with diabetes mellitus.

- People who abuse alcohol.

- Smokers.

- Healthcare workers.

The settings of interest were primary healthcare facilities and other community settings.

We excluded studies that selected participants for enrolment based on the results of prior tuberculosis testing, such as symptom screening or chest radiography.

\section{Index tests}

The index test were sputum Xpert MTB/RIF and sputum Xpert Ultra. Test results are automatically generated (i.e. there is a single threshold), and the user is provided with a printable test result as follows.

\section{Xpert MTB/RIF (Cepheid 2019)}

- MTB (M tuberculosis) DETECTED; RIF (rifampicin) Resistance DETECTED

- MTB DETECTED; RIF Resistance NOT DETECTED

- MTB detected; RIF Resistance INDETERMINATE

- MTB NOT DETECTED.

- INVALID (the presence or absence of MTB cannot be determined)

- ERROR (the presence or absence of MTB cannot be determined)

- NO RESULT (the presence or absence of MTB cannot be determined

Xpert Ultra (Cepheid 2018)

- MTB (M tuberculosis) DETECTED HIGH; RIF (rifampicin) Resistance DETECTED

- MTB DETECTED MEDIUM; RIF Resistance DETECTED

- MTB DETECTED LOW; RIF Resistance DETECTED

- MTB DETECTED VERY LOW; RIF Resistance DETECTED

- MTB DETECTED HIGH; RIF Resistance NOT DETECTED

- MTB DETECTED MEDIUM; RIF Resistance NOT DETECTED

- MTB DETECTED LOW; RIF Resistance NOT DETECTED

- MTB DETECTED VERY LOW; RIF Resistance NOT DETECTED

- MTB DETECTED HIGH; RIF Resistance INDETERMINATE

- MTB DETECTED MEDIUM; RIF Resistance INDETERMINATE

- MTB DETECTED LOW; RIF Resistance INDETERMINATE

- MTB DETECTED VERY LOW; RIF Resistance INDETERMINATE

- MTB Trace DETECTED; RIF Resistance INDETERMINATE

- INVALID (the presence or absence of MTB cannot be determined)

- ERROR (the presence or absence of MTB cannot be determined)

- NO RESULT (the presence or absence of MTB cannot be determined)

Xpert Ultra incorporates a semi-quantitative classification for results. MTB Trace DETECTED corresponds to the lowest bacterial burden for detection of $M$ tuberculosis (Chakravorty 2017). We considered a trace result to mean MTB ( $M$ tuberculosis) DETECTED.

Xpert MTB/RIF and Xpert Ultra assays for screening for pulmonary tuberculosis and rifampicin resistance in adults, irrespective of signs

Copyright (c) 2021 The Authors. Cochrane Database of Systematic Reviews published by John Wiley \& Sons, Ltd. on behalf of The Cochrane

Collaboration. 
However, no rifampicin-resistance results were available for participants with trace results because for trace results, rifampicin resistance is always reported as INDETERMINATE (Cepheid 2018).

\section{Target conditions}

The target conditions were active pulmonary tuberculosis and rifampicin resistance.

\section{Reference standards}

For tuberculosis, the reference standards were solid culture or automated liquid culture.

For rifampicin resistance, the reference standards were culturebased drug susceptibility testing (DST) and line probe assays (WHO LPA 2016). Acceptable methods for DST included the proportion method, performed on solid media, such as Lowenstein-Jensen, and use of a commercial liquid culture system, such as Mycobacteria Growth Indicator Tube (MGIT) 960 automated mycobacterial detection system (BD, USA).

\section{Search methods for identification of studies}

We attempted to identify all relevant studies regardless of language or publication status (published, unpublished, in press, and ongoing).

\section{Electronic searches}

We searched the following databases on 19 March 2020, without language restriction, using the search terms and strategy described in Appendix 1.

- Cochrane Infectious Diseases Specialized Register.

- MEDLINE (Pubmed, from 1966).

- Embase (OVID, from 1947).

- Science Citation Index - Expanded (from 1900), Conference Proceedings Citation Index - Science (CPCI-S, from 1990), Social Science Citation Index (from 1900), Conference Proceedings Citation Index-Social Science \& Humanities(from 1990), all from the Web of Science.

- Scopus (Elsevier, from 1970).

- Latin American Caribbean Health Sciences Literature (LILACS; BIREME, https://lilacs.bvsalud.org/en/ from 1982).

We also searched ClinicalTrials.gov, the WHO International Clinical Trials Registry Platform (ICTRP; www.who.int/trialsearch), and the International Standard Randomized Controlled Trials Number (ISRCTN) registry (www.isrctn.com/), for trials in progress, and ProQuest Dissertations \& Theses A\&I (from 1990) for dissertations.

\section{Searching other resources}

We reviewed reference lists of included articles, related Cochrane Reviews (Horne 2019) and any relevant review articles identified through the above methods. We also contacted researchers at the Foundation for Innovative New Diagnostics (FIND), the WHO Global TB Programme, and other experts in the field of tuberculosis diagnostics for information on ongoing and unpublished studies.

\section{Data collection and analysis}

\section{Selection of studies}

We used Covidence to manage the selection of studies (Covidence). Two review authors independently and in parallel scrutinized titles and abstracts identified from literature searching to identify potentially eligible studies. We retrieved the article of any citation, identified by any review author, for full-text review. Two review authors independently and in parallel assessed articles for inclusion using the predefined selection criteria. We resolved any discrepancies by discussion or with a third review author. We recorded all studies excluded after full-text assessment, along with our reasons for their exclusion in the Characteristics of excluded studies table, and illustrated the study selection process in a PRISMA diagram (Moher 2009).

\section{Data extraction and management}

We extracted data on the following characteristics.

- Author, publication year, study design, country where study was located, clinical setting.

- Population characteristics: age, sex, AFB smear status, HIV status.

- Index test(s), Xpert MTB/RIF or Xpert Ultra.

- Reference standard.

- Quality Assessment of Studies of Diagnostic Accuracy - Revised (QUADAS-2) items (Whiting 2011).

- Number of TP, FP, FN, and TN (i.e. true positives, false positives, false negatives, and true negatives) and trace results, with respect to culture.

- Number of uninterpretable results for detection of pulmonary tuberculosis.

- Number of indeterminate results for detection of rifampicin resistance.

We classified country income status as either low- and middleincome or high-income, according to the World Bank List of Economies (World Bank 2020). In addition, we classified 'country' as being high burden or not high burden for tuberculosis, TB/HIV, or MDR-TB, according to the classification by the WHO (WHO Global TB Report 2019).

We followed Cochrane policy, which states that "authors of primary studies will not extract data from their own study or studies. Instead, another author will extract these data, and check the interpretation against the study report and any available study registration details or protocol".

\section{Assessment of methodological quality}

We used the QUADAS-2 tool, tailored to this review, to assess the quality of the included studies (Whiting 2011; Appendix 2). QUADAS-2 consists of four domains: patient selection, index test, reference standard, and flow and timing. We assessed all domains for risk of bias and the first three domains for concerns regarding applicability. We presented the results of this quality assessment in text, tables, and graphs.

\section{Statistical analysis and data synthesis}

We performed descriptive analyses for the results of the included studies using Stata 15 (Stata). We determined sensitivity and

Xpert MTB/RIF and Xpert Ultra assays for screening for pulmonary tuberculosis and rifampicin resistance in adults, irrespective of signs 13 or symptoms (Review)

Copyright (c) 2021 The Authors. Cochrane Database of Systematic Reviews published by John Wiley \& Sons, Ltd. on behalf of The Cochrane

Collaboration. 
specificity estimates and 95\% confidence intervals (Cls) for individual studies and generated forest plots using Review Manager 5 (Review Manager 2020).

When possible, we carried out meta-analyses to estimate the pooled sensitivity and specificity of the index tests separately for tuberculosis detection and rifampicin resistance detection. We determined the pooled accuracy estimates using an adaptation of the bivariate random-effects model of Reitsma 2005, which uses the exact binomial likelihood for the observed proportions (Chu 2006). The bivariate random-effects approach allows us to calculate the pooled estimates of sensitivity and specificity while accounting for:

1. variation in sensitivity and specificity estimates within individual studies;

2. correlation between sensitivity and specificity across studies; and

3. variation in sensitivity and specificity between studies.

In addition, we determined positive and negative predictive values at pretest probabilities $(0.5 \%$ and $5 \%)$ suggested by the WHO.

For analysis of Xpert MTB/RIF or Xpert Ultra accuracy for detection of rifampicin resistance, we included participants who:

1. were culture-positive;

2. had a valid phenotypic drug susceptibility test (DST) or line probe assay (LPA) result;

3. were Xpert MTB/RIF or Xpert Ultra tuberculosis-positive; and

4. had a valid Xpert MTB/RIF or Xpert Ultra result for rifampicin resistance, detected or not detected (susceptible).

Sensitivity $=$ Xpert MTB/RIF (or Xpert Ultra) rifampicin resistance detected/phenotypic DST or LPA rifampicin-resistant

Specificity $=$ Xpert MTB $/$ RIF (or Xpert Ultra) rifampicin resistance not detected/phenotypic DST or LPA rifampicin-susceptible

We estimated all models using a Bayesian approach, with lowinformation prior distributions, using OpenBUGS software (Version 3.2.3; Lunn 2009), along with $R$ (Version 3.3.2; R Core Team 2019). Under the Bayesian approach, all unknown parameters must be provided a prior distribution that defines the range of possible values of the parameter and the likelihood of each of those values based on information external to the data. In order to let the observed data determine the final results, we chose to use lowinformation prior distributions over the pooled sensitivity and specificity parameters and their between-study standard deviation parameters.

Meta-analysis models can be sensitive to the choice of prior distributions over between-study standard deviation parameters. We therefore carried out sensitivity analyses and considered alternative prior distributions that are less informative, allowing a wider range of possible values. We included information from the prior distribution in combination with the observed data in accordance with Bayes' theorem to obtain a posterior distribution for each unknown parameter.

Using a sample from the posterior distribution, we can obtain various descriptive statistics of interest. We estimated the median pooled sensitivity and specificity and their $95 \%$ credible intervals (Crls). The median or the $50 \%$ quantile is the value below which lies $50 \%$ of the posterior sample. We reported the median because the posterior distributions of some parameters may be skewed and the median would be considered a better point estimate of the unknown parameter than the mean in such cases. The $95 \% \mathrm{Crl}$ is the Bayesian equivalent of the classical (frequentist) $95 \% \mathrm{Cl}$. (We indicated $95 \% \mathrm{Cl}$ for individual study estimates and 95\% Crl for pooled study estimates, as appropriate.) The 95\% Crl may be interpreted as an interval that has a $95 \%$ probability of capturing the true value of the unknown parameter, given the observed data and the prior information. We generated bivariate plots of the credible and prediction regions in the receiver operating characteristic (ROC) space using R (version 3.3.2; R Core Team 2019).

We found only one study that compared the accuracy of Xpert MTB/ RIF and Xpert Ultra in a single high-risk group and setting, thus we analysed the accuracy estimates descriptively in text, tables, and forest plots.

\section{Approach to uninterpretable index test results}

The index tests report an uninterpretable test result for unexpected results with any of the internal control measures of the assay.

In previous reviews, we found very few uninterpretable results reported, as was the case here, and chose to exclude them from the bivariate meta-analyses (Horne 2019).

\section{Investigations of heterogeneity}

We visually inspected forest plots and the summary receiver operating characteristic (SROC) plots for heterogeneity. We set out to investigate a number of potential sources of heterogeneity, described below using subgroup analyses; however, our ability to investigate these sources was limited by the available data. We added percentage of participants with tuberculosis symptoms as a continuous covariate on forest plots and visually inspected the plots. We intended to perform subgroup analyses among studies conducted in high versus not high tuberculosis burden countries, and similarly for high TB/HIV burden and high MDR-TB burden versus not high-burden countries. However, most studies were conducted in high-burden countries (Differences between protocol and review).

\section{Sensitivity analyses}

We intended to perform sensitivity analyses by limiting inclusion in the meta-analyses according to the following criteria:

- studies that explicitly represented the use of the index tests for the screening of individuals irrespective of signs and symptoms of tuberculosis;

- studies that used liquid culture as the reference standard;

- studies where a consecutive or random sample of participants were enrolled. We planned to exclude studies where we answered no or unclear to the QUADAS-2 patient selection signalling question: "Could the selection of patients have introduced bias?"

However, we did not perform any sensitivity analyses because all studies met these criteria (Differences between protocol and review).

Xpert MTB/RIF and Xpert Ultra assays for screening for pulmonary tuberculosis and rifampicin resistance in adults, irrespective of signs 


\section{Assessment of reporting bias}

We did not formally assess reporting bias using funnel plots or regression tests as these have not been reported as helpful for diagnostic test accuracy studies (Macaskill 2010).

\section{Summary of findings and assessment of the certainty of the evidence}

We assessed the certainty of the evidence using the GRADE approach (Balshem 2011; Schünemann 2008; Schünemann 2016), and GRADEpro GDT 2020 software. In the context of a systematic review, ratings of the certainty of the evidence reflect the extent of our confidence that the estimates of effect (including test accuracy and associations) are correct. As recommended, we rated the certainty of the evidence as either high (not downgraded), moderate (downgraded by one level), low (downgraded by two levels), or very low (downgraded by more than two levels) for five domains: risk of bias, indirectness, inconsistency, imprecision, and publication bias.

For each outcome, we considered the certainty of the evidence to begin as high when high-quality observational studies (crosssectional or cohort studies) enrolled participants with diagnostic uncertainty. If we had a reason for downgrading, we used our judgement to classify the reason as serious (downgraded by one level) or very serious (downgraded by two levels). We summarized this information in the 'Summary of findings' tables.

As recommended, we applied GRADE in the following ways (Schünemann 2020a; Schünemann 2020b).

- Risk of bias: we used QUADAS-2 to assess risk of bias.

- Indirectness: we assessed indirectness in relation to the population (including disease spectrum), setting, interventions, and outcomes (accuracy measures). For example, we noted whether the population was the same in the studies compared to the question asked. We also used prevalence as a guide to whether there was indirectness in the population.

- Inconsistency: GRADE recommends downgrading for unexplained inconsistency in sensitivity and specificity estimates. We carried out prespecified analyses and downgraded only when we could not explain inconsistency in the accuracy estimates.

- Imprecision: we considered a precise estimate to be one that would allow a clinically meaningful decision. We considered the width of the $\mathrm{Crl}$ and ask ourselves, 'Would we make a different decision if the lower or upper boundary of the $\mathrm{Crl}$ represented the truth?' In addition, we determined projected ranges for true positives (TP), false negatives (FN), true negatives (TN), and false positives (FP) for a given prevalence of tuberculosis and make judgements on imprecision from these calculations.

- Publication bias: we considered the comprehensiveness of the literature search and outreach to researchers in tuberculosis, the presence of only studies that produce precise estimates of high accuracy despite small sample size, and knowledge about studies that were conducted, but are not published.

\section{RES U L T S}

\section{Results of the search}

We identified 1794 records through database searching and one additional record through other sources. After duplicate removal, we screened a total of 1792 citations by title and abstract for inclusion. Of these, we assessed 119 full-text publications against our inclusion criteria and excluded 99 publications. Exclusions were mainly due to persons not screened irrespective of symptoms ( $\mathrm{n}$ $=61)$, no microbiologic reference standard $(n=14)$, duplicate data from another study $(n=7)$, and data insufficient for the $2 \times 2$ table $(n=6)$. Other reasons for exclusion included Xpert MTB/RIF used on a non-respiratory specimen $(n=3)$, paediatric population $(n=$ 3 ), data not disaggregated on persons screened with or regardless of symptoms $(n=2)$, not original research $(n=2)$, and number of positive tests not reported $(n=1)$.

Thus we identified 20 publications, which included 21 unique studies (one publication contributed two distinct cohorts). (AlDarraji 2013; Al-Darraji 2016; Balcha 2014; Beyanga 2018; Bjerrum 2016; Dorman 2012; Heidebrecht 2016; Henostroza 2016; Kempker 2019; LaCourse 2016; Lawn 2011; Lawn 2012; Lopez-Varela 2019; Mollel 2017; Ntinginya 2012; O'Grady 2012; Reeve 2019a; Reeve 2019b; Santos 2020; Tahseen 2018; Yoon 2017). Of the total 21 studies, 18 studies provided data for the detection of pulmonary tuberculosis using Xpert MTB/RIF and one study provided data for both Xpert MTB/RIF and Xpert Ultra (Reeve 2019b). Three studies provided data for detection of rifampicin resistance (AlDarraji 2013; Lawn 2011; O'Grady 2012). All included studies used a cross-sectional study design. We did not identify any studies that conducted general population-wide screening for tuberculosis (e.g. national prevalence surveys) that met inclusion criteria for this review. Figure 2 shows the flow of studies in the review. We recorded the excluded studies and the reasons for their exclusion in the Characteristics of excluded studies table. 
Figure 2. Study flow diagram, PRISMA. *One publication, Reeve 2019 , contributed two distinct studies, which were classified as Reeve 2019a and Reeve 2019b.

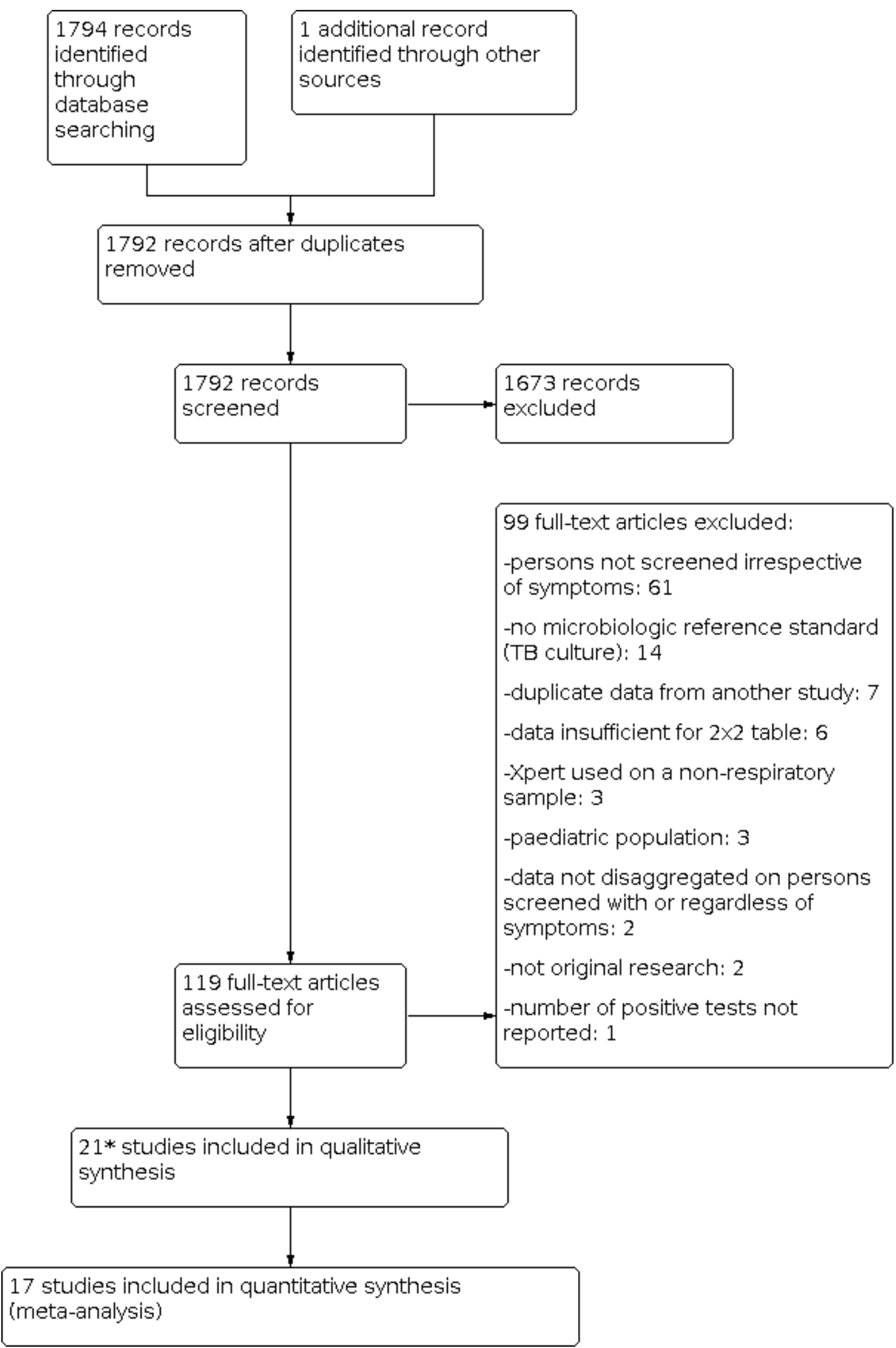




\section{Methodological quality of included studies}

\section{Xpert MTB/RIF and Xpert Ultra as screening tests for}

\section{pulmonary tuberculosis}

Figure 3 and Figure 4 summarize risk of bias and applicability concerns for studies evaluating Xpert MTB/RIF $(n=20)$ and Xpert Ultra $(n=1)$ as screening tests for pulmonary tuberculosis.

Figure 3. Risk of bias and applicability concerns graph for Xpert MTB/RIF and Xpert Ultra as screening tests for pulmonary tuberculosis: review authors' judgements about each domain presented as percentages across included studies.

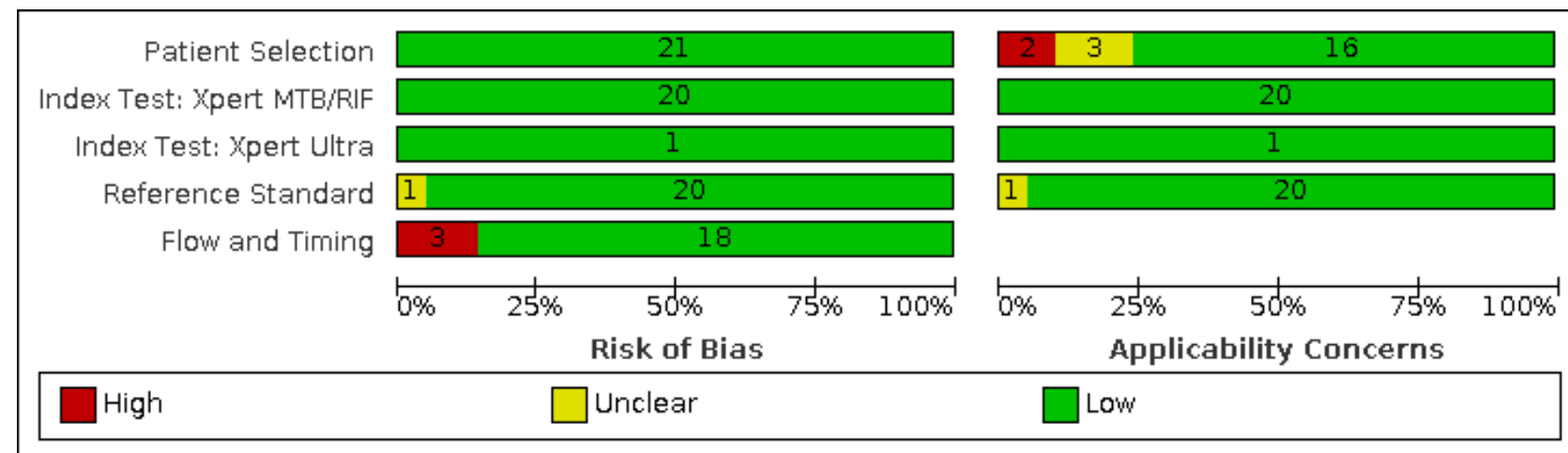


Figure 4. Risk of bias and applicability concerns summary for Xpert MTB/RIF and Xpert Ultra as screening tests for pulmonary tuberculosis: review authors' judgements about each domain for each included study.

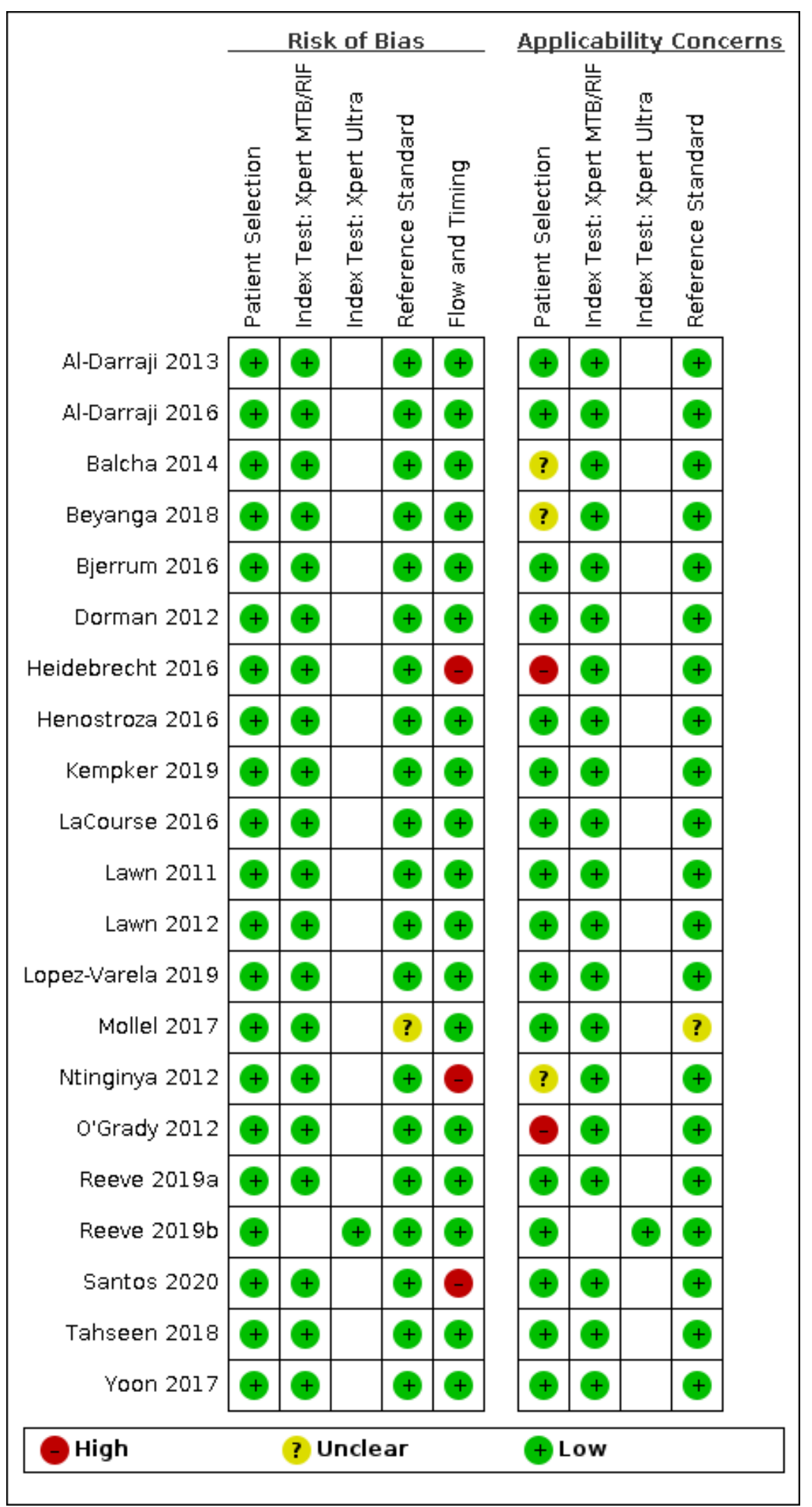

Xpert MTB/RIF and Xpert Ultra assays for screening for pulmonary tuberculosis and rifampicin resistance in adults, irrespective of signs 
In the patient selection domain, we considered all studies to have low risk of bias because the studies enrolled a consecutive or random sample of eligible adult participants and avoided inappropriate exclusions. Regarding applicability (Patient Selection domain), we considered 16 studies (76\%) to have low concern because the study population resembled a population that was selected for tuberculosis screening in community settings or primary care centres. We considered two studies $(10 \%)$ to have high concern because participants were evaluated exclusively as inpatients in tertiary care centres (Heidebrecht 2016; O'Grady 2012), and three studies (14\%) to have unclear concern, two studies because they enrolled a small proportion of people younger than 15 years old (Beyanga 2018; Ntinginya 2012), and one study because $2 \%$ of the enrolled population had received tuberculosis treatment for up to two weeks (Balcha 2014).

In the index test domain, we considered all studies to have low risk of bias because the results of the index tests (Xpert MTB/RIF and Xpert Ultra) are automatically generated, the user is provided with printable test results, and the positivity threshold is prespecified. Regarding applicability (Index Test domain), we considered all studies to have low concern.
In the reference standard domain, we considered 20 studies (95\%) to have low risk of bias. We considered one study to have unclear risk of bias because information about blinding was not reported (Mollel 2017). Regarding applicability (Reference Standard domain), we considered 20 studies (95\%) to have low concern because these studies performed a test to identify $M$ tuberculosis species (speciation) and one study to have unclear concern because information about speciation was not reported (Mollel 2017).

In the flow and timing domain, we considered 18 studies (86\%) to have low risk of bias because all participants were included in the analysis. We considered three studies to have high risk of bias because not all enrolled participants were included in the analysis (Heidebrecht 2016; Ntinginya 2012; Santos 2020).

\section{Xpert MTB/RIF and Xpert Ultra as screening tests for rifampicin resistance}

Figure 5 and Figure 6 show risk of bias and applicability concerns for studies evaluating Xpert MTB/RIF $(n=3)$ as screening tests for rifampicin resistance.

Figure 5. Risk of bias and applicability concerns graph for Xpert MTB/RIF as a screening test for rifampicin resistance: review authors' judgements about each domain presented as percentages across included studies.

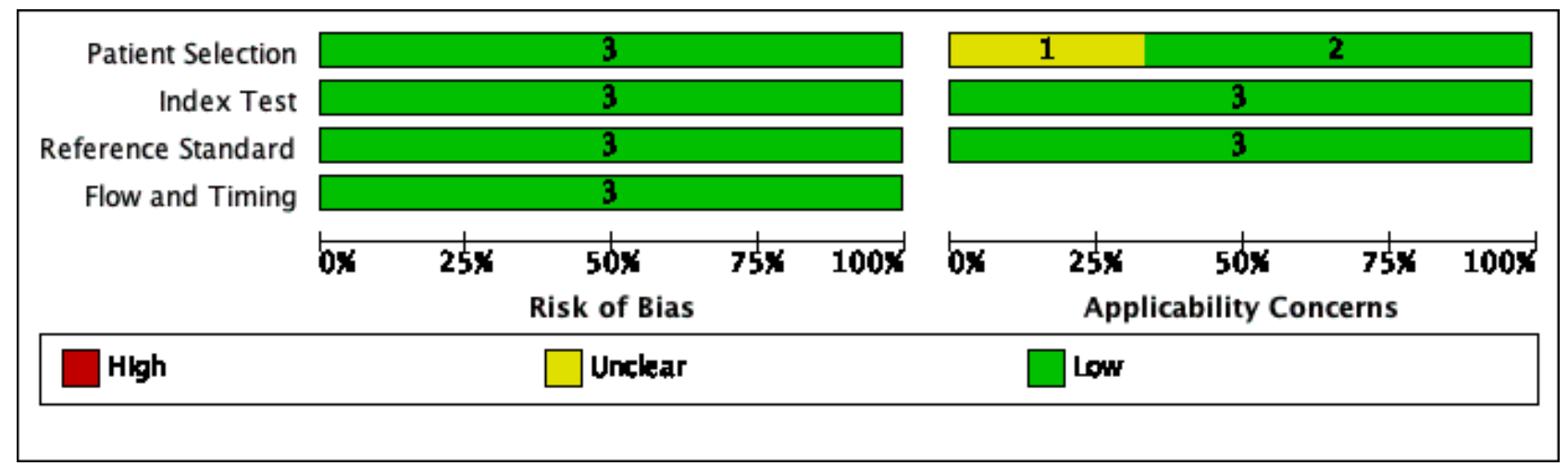


Figure 6. Risk of bias and applicability concerns summary for Xpert MTB/RIF as a screening test for rifampicin resistance: review authors' judgements about each domain for each included study.

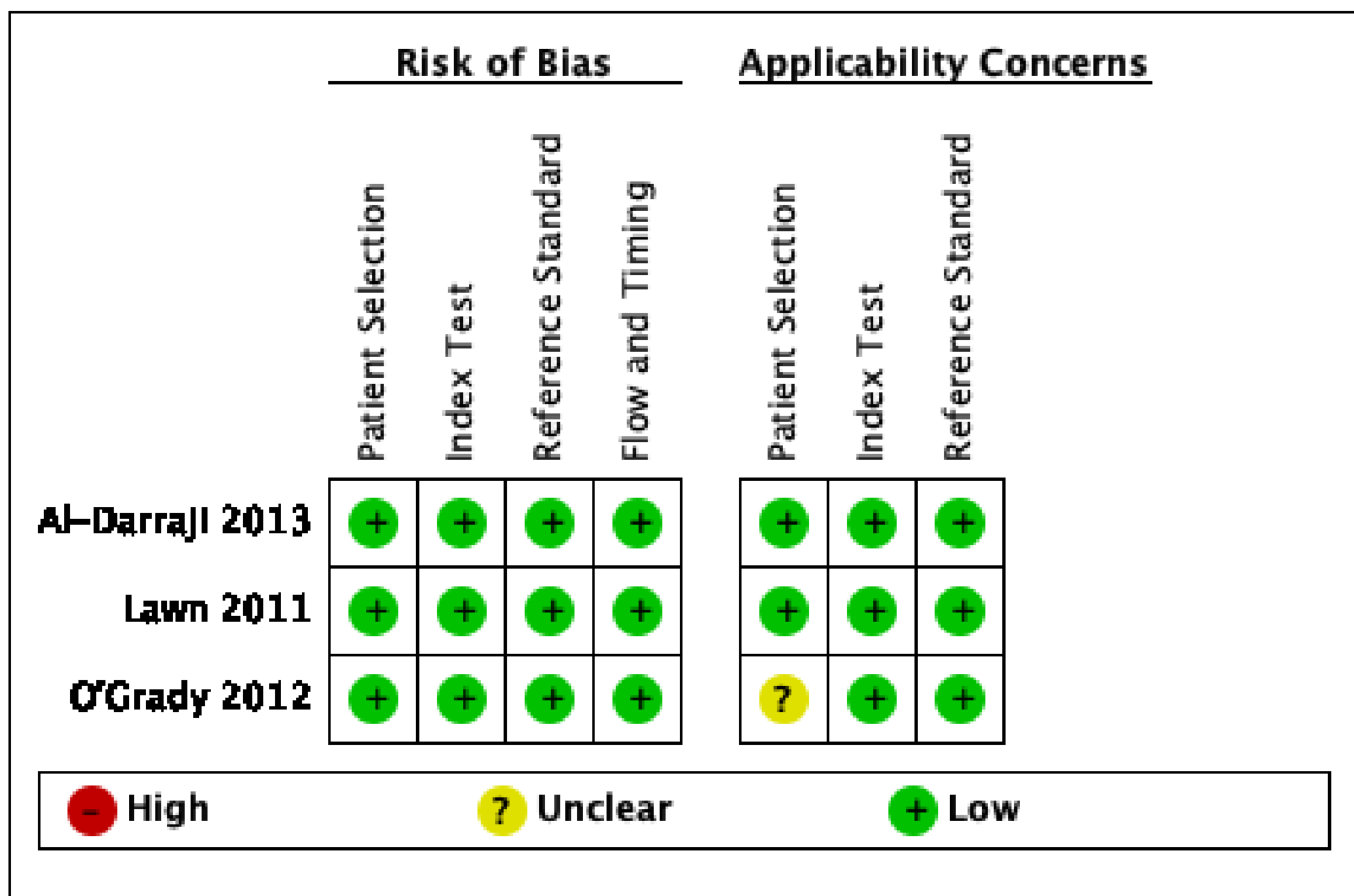

Regarding risk of bias, for the four domains (patient selection, index test, reference standard, and flow and timing), we considered all three studies (100\%) to at low risk (Al-Darraji 2013; Lawn 2011; O'Grady 2012). Regarding applicability, in the Patient Selection domain, we considered two studies (67\%) to have low concern about applicability (Al-Darraji 2013; Lawn 2011), and one study to have unclear concern because participants were evaluated exclusively as inpatients in a tertiary care centre (O'Grady 2012).

\section{Findings}

The median study population size of the included studies was 442 (Interquartile range (IQR) 114 to 624). Fifteen studies (75\%) were conducted in high tuberculosis burden and $16(80 \%)$ in high TB/HIVburden countries. We presented key characteristics of the included studies in the Characteristics of included studies table. Twelve $(60 \%)$ studies were performed in people living with HIV. Of the total 21 studies, none evaluated the tests for screening in the general population.
Xpert MTB/RIF and Xpert Ultra as screening tests for pulmonary tuberculosis

Xpert MTB/RIF as a screening test in people living with HIV, irrespective of tuberculosis symptoms

Twelve studies evaluated Xpert MTB/RIF as a screening test for pulmonary tuberculosis in people living with HIV (Al-Darraji 2013; Balcha 2014; Bjerrum 2016; Henostroza 2016; Kempker 2019; LaCourse 2016; Lawn 2012; Lopez-Varela 2019; Mollel 2017; Reeve 2019a; Tahseen 2018; Yoon 2017). Xpert MTB/RIF sensitivity estimates varied from $43 \%$ to $100 \%$. The lowest sensitivity was reported by LaCourse 2016 , a study notable for enrolling HIV-positive women accessing prevention of mother-tochild transmission services as part of antenatal care. Specificity varied less than sensitivity, from $92 \%$ to $100 \%$, Figure 7. Xpert MTB/ RIF pooled sensitivity and specificity $(95 \% \mathrm{Crl}$ ) were $61.8 \%$ (53.6 to 69.9 ) and $98.8 \%$ (98.0 to 99.4 ), (12 studies, 4775 participants, 602 (12.6\%) with tuberculosis), Table 1, Figure 8. 
Figure 7. Forest plots of Xpert MTB/RIF sensitivity and specificity for pulmonary tuberculosis in people living with HIV by percentage of tuberculosis symptoms. The individual studies are ordered by decreasing percentage of participants with tuberculosis symptoms. The squares represent the sensitivity and specificity of one study, the black line its confidence interval. TP: true-positive; FP: false-positive; FN: false-negative; TN: true-negative.

Xpert MTB/RIF, HIV positive, irrespective of TB symptoms

Study

Lawn 2012

Yoon 2017

Henostroza 2016

Balcha 2014

Al-Darraji 2013

Kempker 2019

Mollel 2017

Bjerrum 2016

Reeve 2019a

Lopez-Varela 2019

Tahseen 2018

$\begin{array}{rrrr}\text { TP } & \text { FP } & \text { FN } & \text { TN } \\ 49 & 4 & 36 & 427 \\ 84 & 8 & 79 & 1006 \\ 39 & 5 & 23 & 256 \\ 81 & 13 & 41 & 677 \\ 8 & 0 & 7 & 110 \\ 9 & 3 & 3 & 88 \\ 9 & 0 & 0 & 60 \\ 27 & 5 & 8 & 55 \\ 33 & 1 & 35 & 502 \\ 3 & 0 & 1 & 87 \\ 13 & 11 & 7 & 574 \\ 3 & 1 & 4 & 280\end{array}$

Percentage

aCourse 2016

34280

Xpert Ultra, HIV, irrespective of TB symptoms

Study TP FP FN TN

$\begin{array}{lllll}\text { Reeve 2019b } & 47 \quad 9 & 21 & 494\end{array}$

$\begin{array}{rrr}\text { ptoms } & \text { Sensitivity }(95 \% \mathrm{Cl}) & \text { Specificity }(95 \% \mathrm{Cl}] \\ 90.0 & 0.58[0.46,0.68] & 0.99[0.98,1.00] \\ 87.0 & 0.52[0.44,0.59] & 0.99[0.98,1.00] \\ 86.0 & 0.63[0.50,0.75] & 0.98[0.96,0.99] \\ 80.0 & 0.66[0.57,0.75] & 0.98[0.97,0.99] \\ 68.0 & 0.53[0.27,0.79] & 1.00[0.97,1.00] \\ 63.0 & 0.75[0.43,0.95] & 0.97[0.91,0.99] \\ 60.0 & 1.00[0.66,1.00] & 1.00[0.94,1.00] \\ 60.0 & 0.77[0.60,0.90] & 0.92[0.82,0.97 \\ 52.0 & 0.49[0.36,0.61] & 1.00[0.99,1.00] \\ 41.0 & 0.75[0.19,0.99] & 1.00[0.96,1.00 \\ 30.0 & 0.65[0.41,0.85] & 0.98[0.97,0.99 \\ 19.0 & 0.43[0.10,0.82] & 1.00[0.98,1.00]\end{array}$

$0.43[0.10,0.82]$

$1.00[0.98,1.00]$

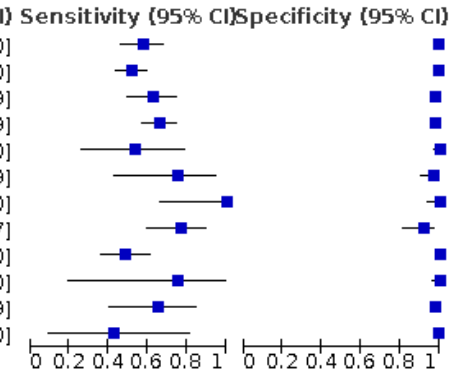

Sensitivity (95\% Cl]Specificity $(95 \% \mathrm{Cl})$

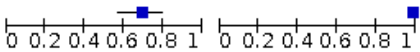


Figure 8. Summary plots of the accuracy of Xpert MTB/RIF as a screening test for pulmonary tuberculosis in (A) people living with HIV and (B) non-hospitalized people in high-risk groups. Each individual study is represented by a shaded circle. The size of the circle is proportional to the sample size of the study such that larger studies are represented by larger circles. The filled circle is the median pooled estimate for sensitivity and specificity. The solid lines represent the $95 \%$ credible region around the summary estimate; the dashed lines represent the $95 \%$ prediction region. The range is truncated to consider only those regions of the ROC space where data have been observed.

(A) Xpert MTB/RIF in people living with HIV

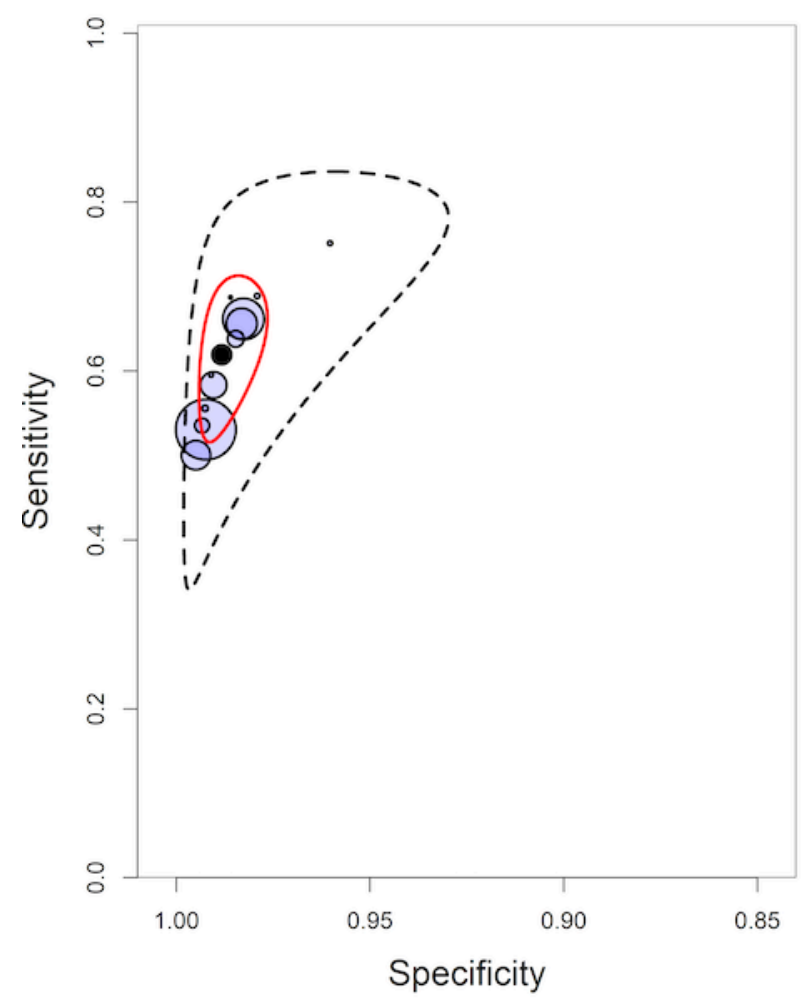

Investigations of heterogeneity

Xpert MTB/RIF as a screening test in people living with HIV, by percentage of participants with tuberculosis symptoms

In HIV-positive populations where $50 \%$ or more had tuberculosis symptoms, Xpert MTB/RIF pooled sensitivity and specificity (95\% Crl) were $62.9 \%$ (53.9 to 72.1 ) and $98.7 \%$ (97.7 to 99.4), (9 studies, 3791 participants, 571 (15.1\%) with tuberculosis).

In HIV-positive populations where less than $50 \%$ had tuberculosis symptoms, Xpert MTB/RIF pooled sensitivity and specificity (95\% Crl) were $61.1 \%$ (35.5 to 82.3 ) and $99.1 \%$ (97.6 to 99.8), (3 studies, 984 participants, 31 (3.2\%) with tuberculosis).

Confidence intervals for sensitivity and specificity estimates in the two subgroups overlapped, indicating no significant differences in accuracy based on tuberculosis symptoms, Table 1, Figure 7.
(B) Xpert MTB/RIF in non-hospitalized people in high-risk groups

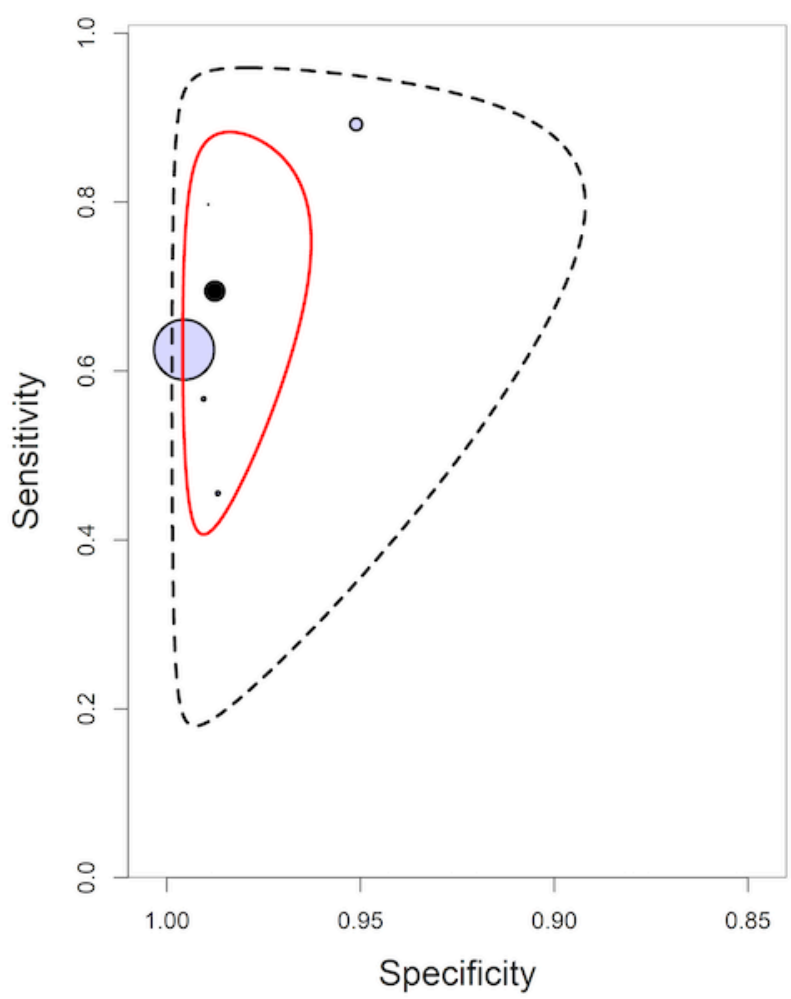

Xpert Ultra as a screening test in people living with HIV, irrespective of tuberculosis symptoms

One study evaluated Xpert Ultra as a screening test for pulmonary tuberculosis (Reeve 2019b). Xpert Ultra sensitivity and specificity $(95 \% \mathrm{Cl})$ were $69 \%$ (57 to 80 ) and $98 \%$ (97 to 99$)$, Figure 7.

Xpert MTB/RIF as a screening test in household contacts, irrespective of tuberculosis symptoms

Two studies evaluated Xpert MTB/RIF as a screening test for pulmonary tuberculosis in household contacts. Xpert MTB/RIF sensitivity and specificity $(95 \% \mathrm{Cl})$ were $33 \%$ (13 to 59$)$ and $98 \%(97$ to 99) (Beyanga 2018) and 100\% (48 to 100) and 100\% (93 to 100) (Ntinginya 2012), Figure 9. 
Figure 9. Forest plots of Xpert MTB/RIF sensitivity and specificity for pulmonary tuberculosis in household contacts, people in prison, miners, and people admitted to hospital, irrespective of tuberculosis symptoms. The squares represent the sensitivity and specificity of one study, the black line its confidence interval. TP: true-positive; FP: false-positive; FN: false-negative; TN: true-negative.

Xpert MTB/RIF, household contacts, irrespective of TB symptoms

$\begin{array}{lrrrrrr}\text { Study } & \text { TP } & \text { FP } & \text { FN } & \text { TN } & \text { Sensitivity }(95 \% \mathrm{Cl}) & \text { Specificity }(95 \% \mathrm{Cl}) \\ \text { Ntinginya 2012 } & 5 & 0 & 0 & 51 & 1.00[0.48,1.00] & 1.00[0.93,1.00] \\ \text { Beyanga 2018 } & 6 & 7 & 12 & 427 & 0.33[0.13,0.59] & 0.98[0.97,0.99]\end{array}$

Xpert MTB/RIF, prisoners, irrespective of TB symptoms

$\begin{array}{lrrrrrr}\text { Study } & \text { TP } & \text { FP } & \text { FN } & \text { TN } & \text { Sensitivity }(95 \% \mathrm{Cl}) & \text { Specificity }(95 \% \mathrm{Cl}) \\ \text { Santos 2020 } & 88 & 65 & 9 & 1223 & 0.91[0.83,0.96] & 0.95[0.94,0.96] \\ \text { Al-Darraji 2016 } & 16 & 4 & 14 & 408 & 0.53[0.34,0.72] & 0.99[0.98,1.00]\end{array}$

Xpert MTB/RIF, miners, irrespective of TB symptoms

$\begin{array}{lrrrrrr}\text { Study } & \text { TP } & \text { FP } & \text { FN } & \text { TN } & \text { Sensitivity }(95 \% \text { Cl) } & \text { Specificity }(95 \% \text { Cl) } \\ \text { Dorman } 2012 & 117 & 27 & 70 & 6407 & 0.63[0.55,0.70] & 1.00[0.99,1.00]\end{array}$

Xpert MTB/RIF, admitted patients, irrespective of TB symptoms

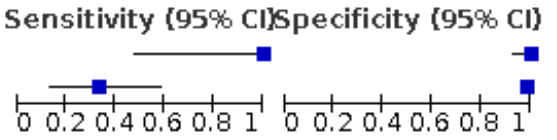

Sensitivity $(95 \%$ Cl)Specificity $(95 \% \mathrm{Cl})$



Sensitivity (95\% Cl)Specificity (95\% Cl)

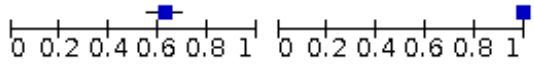

$\begin{array}{lrrrrrrr}\text { Study } & \text { TP } & \text { FP } & \text { FN } & \text { TN } & \text { Sensitivity }(95 \% \mathrm{Cl}) & \text { Specificity }(95 \% \mathrm{Cl}) \text { Sensitivity }(95 \% \mathrm{Cl}) \text { Specificity }(95 \% \mathrm{Cl}) \\ \text { O'Grady } 2012 & 173 & 22 & 28 & 420 & 0.86[0.80,0.91] & 0.95[0.93,0.97] & \end{array}$

Xpert MTB/RIF as a screening test in people residing in prisons, irrespective of tuberculosis symptoms

Two studies evaluated Xpert MTB/RIF as a screening test for pulmonary tuberculosis in persons residing in prisons. Xpert MTB/ RIF sensitivity and specificity $(95 \% \mathrm{Cl}$ ) were $53 \%$ (34 to 72 ) and $99 \%$ (98 to 100) (Al-Darraji 2016) and 91\% (83 to 96) and 95\% (94 to 96) (Santos 2020), Figure 9.

\section{Xpert MTB/RIF as a screening test in miners, irrespective of tuberculosis symptoms}

One study evaluated Xpert MTB/RIF as a screening test for pulmonary tuberculosis in miners (Dorman 2012). Xpert MTB/RIF sensitivity and specificity $(95 \% \mathrm{Cl})$ were $63 \%$ (55 to 70$)$ and $100 \%$ (99 to 100), Figure 9.

\section{Xpert MTB/RIF as a screening test in non-hospitalized people in high-risk groups combined, irrespective of tuberculosis symptoms}

We estimated pooled sensitivity and specificity including the five studies that evaluated Xpert MTB/RIF in household contacts, miners, and people residing in prisons (i.e. populations that did not exclusively include people living with HIV and inpatient settings) (Al-Darraji 2016; Beyanga 2018; Dorman 2012; Ntinginya 2012; Santos 2020). Xpert MTB/RIF pooled sensitivity and specificity ( $95 \%$ Crl) were $69.4 \%$ (47.7 to 86.2 ) and $98.8 \%$ (97.2 to 99.5 ), (5 studies, 8956 participants, 337 (3.8\%) with tuberculosis), Table 1, Figure 8.

\section{Xpert MTB/RIF as a screening test in patients admitted to the hospital, irrespective of tuberculosis symptoms}

Two studies evaluated Xpert MTB/RIF as a screening test for pulmonary tuberculosis in persons admitted to the hospital. Xpert MTB/RIF sensitivity and specificity $(95 \% \mathrm{Cl}$ ) were $79 \%$ (60 to 92 ) and $95 \%$ (88 to 98 ) (Heidebrecht 2016) and $86 \%$ ( 80 to 91 ) and $95 \%$ (93 to 97) (O'Grady 2012), Figure 9. In Heidebrecht $2016,62 \%$ of patients had HIV and in O'Grady 2012, $71 \%$ of patients had HIV.

\section{Xpert MTB/RIF and Xpert Ultra as a screening test in the general population, irrespective of tuberculosis symptoms}

We did not identify any studies that evaluated Xpert MTB/RIF or Xpert Ultra as a screening test in general populations, irrespective of signs or symptoms of tuberculosis.

\section{Xpert MTB/RIF and Xpert Ultra as screening tests for rifampicin resistance}

\section{Xpert MTB/RIF as a screening test for rifampicin resistance}

Three studies evaluated Xpert MTB/RIF as a screening test for rifampicin resistance (Al-Darraji 2013; Lawn 2011; O'Grady 2012). One study reported zero rifampicin-resistant results and hence, sensitivity was not estimable (Al-Darraji 2013). Sensitivity (95\% Cl) was $100 \%$ (40 to 100) in Lawn 2011 and $81 \%$ (54 to 96) in O'Grady 2012; specificity ranged from 94\% to $100 \%$, Figure 10 . 
Figure 10. Forest plots of Xpert MTB/RIF sensitivity and specificity for rifampicin resistance, in people irrespective of tuberculosis symptoms. The squares represent the sensitivity and specificity of one study, the black line its confidence interval. TP: true-positive; FP: false-positive; FN: false-negative; TN: true-negative.

$\begin{array}{lrrrrr}\text { Study } & \text { TP } & \text { FP } & \text { FN } & \text { TN } & \text { Sensitivity }(95 \% \mathrm{Cl}) \\ \text { Al-Darraji 2013 } & 0 & 0 & 0 & 8 & \text { Not estimable } \\ \text { Lawn 2011 } & 4 & 3 & 0 & 48 & 1.00[0.40,1.00] \\ \text { O'Grady 2012 } & 13 & 2 & 3 & 78 & 0.81[0.54,0.96]\end{array}$

\section{Xpert Ultra as a screening test for rifampicin resistance}

We did not identify any studies that evaluated Xpert Ultra as a screening test for detection of rifampicin resistance.

\section{DISCUSSION}

\section{Summary of main results}

This Cochrane Review summarizes the current literature on the accuracy of Xpert MTB/RIF and Xpert Ultra as screening tests for pulmonary tuberculosis and rifampicin resistance in adults, irrespective of signs and symptoms of tuberculosis. We identified 21 studies: 18 studies (13,114 participants) evaluated Xpert MTB/RIF as a screening test for pulmonary tuberculosis and one study (571 participants) evaluated both Xpert MTB/RIF and Xpert Ultra. Three studies (159 participants) evaluated Xpert MTB/RIF for rifampicin resistance.

- As a screening test for pulmonary tuberculosis in people living with HIV, Xpert MTB/RIF pooled sensitivity and specificity (95\% Crl) were $61.8 \%$ (53.6 to 69.9 ) and $98.8 \%$ (98.0 to 99.4 ), Summary of findings 1.

- As a screening test for pulmonary tuberculosis in people living with HIV (one study), Xpert Ultra sensitivity and specificity (95\% $\mathrm{Cl}$ ) were $69 \%$ (57 to 80 ) and $98 \%$ (97 to 99 ), Summary of findings 1.

- As a screening test for pulmonary tuberculosis in nonhospitalized people in high-risk groups, Xpert MTB/RIF pooled sensitivity and specificity $(95 \% \mathrm{Crl}$ ) were $69.4 \%$ (47.7 to 86.2$)$ and 98.8\% (97.2 to 99.5), Summary of findings 1.

- As a screening test for rifampicin resistance, Xpert MTB/RIF sensitivity was $81 \%$ and $100 \%$, and specificity was $94 \%$ to $100 \%$, Summary of findings 2.

\section{Xpert MTB/RIF as a screening test for pulmonary tuberculosis in people living with HIV}

Results of these studies indicate that, in theory, for a population of 1000 people where 50 have tuberculosis on culture, 40 would be Xpert MTB/RIF-positive; of these, 9 (22\%) would not have tuberculosis (false-positives); and 960 would be Xpert MTB/ RIF-negative; of these, 19 (2\%) would have tuberculosis (falsenegatives), Summary of findings 1.

\section{Xpert Ultra as a screening test for pulmonary tuberculosis in people living with HIV}

Results of these studies indicate that, in theory, for a population of 1000 people where 50 have tuberculosis on culture, 53 would be Xpert Ultra-positive; of these, 19 (36\%) would not have tuberculosis (false-positives); and 947 would be Xpert Ultra-negative; of these,

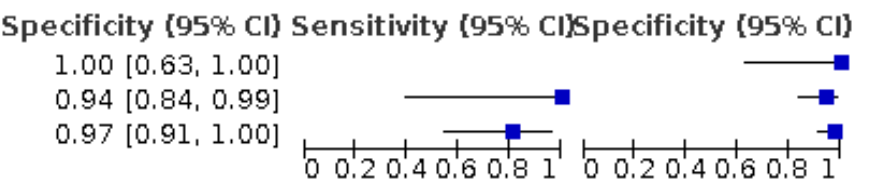

$16(2 \%)$ would have tuberculosis (false-negatives), Summary of findings 1 .

\section{Xpert MTB/RIF as a screening test for pulmonary tuberculosis in non-hospitalized people in high-risk groups}

Results of these studies indicate that, in theory, for a population of 1000 people where 10 have tuberculosis on culture, 19 would be Xpert MTB/RIF-positive; of these, 12 (63\%) would not have tuberculosis (false-positives); and 981 would be Xpert MTB/RIFnegative; of these, 3 ( $0 \%$ ) would have tuberculosis (false-negatives), Summary of findings 1 .

\section{Xpert MTB/RIF and Xpert Ultra as a screening test for pulmonary tuberculosis in high-risk groups}

Our review found that Xpert MTB/RIF and Xpert Ultra, when used as a screening test for tuberculosis, successfully identified tuberculosis in people who were screened, regardless of symptoms of pulmonary tuberculosis in high-risk groups and persons attending health facilities for reasons other than diagnosis of tuberculosis.

The sensitivity and specificity of Xpert MTB/RIF and Xpert Ultra, compared to the microbiological reference standard of tuberculosis culture, was similar in people living with HIV and other high-risk groups, when used to screen for tuberculosis, irrespective of the presence of signs and symptoms.

Current WHO and national guidelines recommend screening people living with HIV for tuberculosis symptoms and reserve diagnostic testing for people with symptoms. In this review, we evaluated two subgroups of studies of people living with HIV: studies in which $50 \%$ or more participants had tuberculosis symptoms, and studies in which less than $50 \%$ of participants had tuberculosis symptoms. While we did not identify any studies using Xpert MTB/RIF to screen entirely asymptomatic people living with HIV for tuberculosis, and are thus unable to estimate Xpert MTB/ RIF accuracy in people living with HIV without symptoms, evidence from the subgroup analysis suggests that there is little difference in the accuracy of the Xpert test in heavily symptomatic versus less symptomatic populations of people living with HIV. The observed prevalence of pulmonary tuberculosis was substantially higher in the high symptom prevalence subgroup of studies compared to the low symptom prevalence subgroup of studies (15.1\% versus $3.2 \%$ ), but the sensitivity and specificity of Xpert MTB/RIF were not meaningfully different in each subgroup.

We recognize that patient-important outcomes including the effect of the use of Xpert MTB/RIF and Xpert Ultra for screening on treatment initiation, cure, mortality, and community incidence are important to patients, clinicians, and decision-makers. However,

Xpert MTB/RIF and Xpert Ultra assays for screening for pulmonary tuberculosis and rifampicin resistance in adults, irrespective of signs 
evaluating such outcomes would have required a different methodology than this study, which focused on test accuracy in the application of screening irrespective of symptoms. We did not identify any studies that assessed both accuracy with a tuberculosis culture reference standard and people-important outcomes. The ACT3 study in Viet Nam (Marks 2019) was a community-randomized trial evaluating the effect of screening for tuberculosis using Xpert MTB/RIF in adults 15 years and older, irrespective of signs and symptoms of tuberculosis, on the prevalence of tuberculosis. Tuberculosis culture was performed on specimens testing positive by Xpert MTB/RIF. The study found 94 positive Xpert MTB/RIF results among 41,680 adults tested in the control arm; tuberculosis culture was positive in 49 of the positive Xpert MTB/RIF results for a positive predictive value of $52 \%$. In the intervention communities of 42,150 adults, only 53 cases were detected by Xpert MTB/ RIF (33 culture-positive), a significant reduction in tuberculosis prevalence. Though we were unable to include this study in our review, as the reference microbiological standard (tuberculosis culture) was not systematically performed with every Xpert MTB/ RIF test, the ACT3 study is an important contribution to the evidence on use of Xpert MTB/RIF in population-based screening and the effect on patient-important outcomes.

There is increasing interest in using Xpert MTB/RIF and Xpert Ultra for population-based screening for tuberculosis, such as for national prevalence surveys. Recent national tuberculosis prevalence surveys including in Vietnam (Nguyen 2020), Kenya (Enos 2018), and Bangladesh (WHO Consolidated Guidelines (Module 3) 2020) employed screening strategies with Xpert MTB/ RIF or Xpert Ultra and reference testing with mycobacterial culture; prevalence surveys in South Africa, Zambia, and Myanmar employed Xpert Ultra and culture for reference testing (WHO Consolidated Guidelines (Module 3) 2020) . However, all prevalence surveys we identified employed Xpert MTB testing only for persons with radiographic signs and/or symptoms of tuberculosis, thus we were unable to determine the screening accuracy of these molecular tests in general population screening in persons irrespective of signs and symptoms of tuberculosis. This is an important limitation of the review.

Decisions of how and where to implement Xpert MTB/RIF and Xpert Ultra for tuberculosis screening in persons irrespective of symptoms require, in addition to concerns of test accuracy as reviewed here, careful consideration of resource utilization requirements and cost-effectiveness of these tests, which are highly dependent on the setting, population, and underlying prevalence of tuberculosis in the population. While there are substantial data supporting the cost-effectiveness for use of Xpert MTB/RIF and Xpert Ultra as an initial diagnostic test in symptomatic individuals presenting to a healthcare facility in high-burden settings (WHO 2016), there is scarce published evidence for cost-effectiveness of these tests when used for screening people irrespective of signs and symptoms of tuberculosis. Communities and populations differ in how they can access rapid molecular diagnostic tests for tuberculosis: when provided in a centralized fashion in a healthcare or other facility-based setting (e.g. primary care clinics providing services to people living with HIV, inpatient hospital facilities, prison facilities, large mining complexes), fewer resources are needed to bring the tests in proximity to people being screened. Screening becomes much more resource-intensive when delivered in decentralized, community-based or household contact-tracing settings, but conversely these settings may be where the majority of undetected tuberculosis cases may be found, and consequently the impact on community transmission the highest.

\section{Strengths and weaknesses of the review}

\section{Completeness of evidence}

The findings in this review are based on comprehensive searching, strict selection criteria, and standardized data extraction. We corresponded with study authors to obtain additional data and information that was missing from the papers. The search strategy included studies published in all languages. We acknowledge that we may have missed studies despite the comprehensive search; however, we think it unlikely that the findings would have changed.

\section{Accuracy of the reference standards used}

Culture is regarded as the best available reference standard for the bacteriological confirmation of pulmonary tuberculosis and was the reference standard for tuberculosis in this review. Liquid culture is considered to be more sensitive than solid culture (Lewinsohn 2017). In this review 17 (85\%) studies used liquid culture as the reference standard.

\section{Quality and quality of reporting of the included studies}

All studies used consecutive or random selection of participants and interpreted the reference standard results without knowledge of index test results. Xpert results are generated automatically, without requiring operator interpretation. Studies were generally well reported, although we corresponded with several authors for missing information.

\section{Applicability of findings to the review question}

For screening for pulmonary tuberculosis, we had low concern for applicability because in most studies, participants represented a population that was selected for tuberculosis screening in community settings or primary care centres. Fifteen studies (75\%) were conducted in high tuberculosis burden settings and hence the results may not be applicable to other settings. We only identified one study that evaluated Xpert Ultra and were therefore unable to compare test accuracy with that of Xpert MTB/RIF, a secondary objective of the review. The one study of Xpert Ultra was conducted in people living with HIV in South Africa, hence applicability to other settings comes with some uncertainty.

For screening for detection of rifampicin resistance detection, of the three included studies we were unclear about the applicability of one study (33\%) because this study evaluated the test in adult medical inpatients at a tertiary hospital, rather than a community setting or primary care centre.

We did not identify any studies that screened other groups at high risk for tuberculosis that met inclusion criteria for this review, which included the concomitant use of culture as a reference standard. These populations include people experiencing homelessness, people with diabetes mellitus, people who abuse alcohol, people who smoke, and healthcare workers. We did not identify any studies that conducted general population-wide screening for tuberculosis (e.g. national prevalence surveys) that met inclusion criteria for this review. This is an important limitation of the review. 


\section{AUTHORS' CONCLUSIONS}

\section{Implications for practice}

Of the high-risk groups evaluated, Xpert MTB/RIF applied as a screening test was accurate for tuberculosis in high tuberculosis burden settings. Sensitivity and specificity were similar in people living with HIV and non-hospitalized people in high-risk groups. In people living with HIV, Xpert Ultra sensitivity was slightly higher than that of Xpert MTB/RIF and specificity similar. As there was only one study of Xpert Ultra in this analysis, results should be interpreted with caution. There were no studies that evaluated the tests in people with diabetes mellitus and other groups considered at high risk for tuberculosis, or in the general population.

\section{Implications for research}

Several high-risk groups were considered that were not represented in any studies in this review, but evidence of the performance of Xpert MTB/RIF, Xpert Ultra, and other rapid molecular tests will be important to inform their use as screening tests for tuberculosis. In particular, priority populations for research are those in whom signs and symptoms of tuberculosis are less sensitive for tuberculosis or in whom the consequences of a missed diagnosis of tuberculosis are particularly severe, such as pregnant women, people with diabetes mellitus, and people who smoke tobacco. Only one study using Xpert Ultra (in people living with HIV) contributed evidence to this review, and additional studies of the accuracy of Xpert Ultra measured against tuberculosis culture in screening people irrespective of symptoms, with particular attention to false-positives, are needed to understand the implications of Xpert Ultra as a screening test. Operational research is needed to optimise the implementation of these tests for use in community settings, ensuring appropriate allocation of resources to enable test delivery, and to understand how use of the tests for screening affects the clinically meaningful outcomes of tuberculosis treatment initiation and cure, and effects on local epidemiology. If data are available, future reviews should assess the accuracy of a class of technologies, rather than a test from a single manufacturer.

\section{ACK N O WLEDGEMENTS}

The Academic Editors are Professor Gerry Davies (Cochrane Infectious Diseases Group (CIDG)) and Dr Stewart Walsh (DTA).

The CIDG editorial base is funded by UK aid from the UK government for the benefit of low- and middle-income countries (project number 300342-104). The views expressed do not necessarily reflect the UK government's official policies.

We are grateful to Vittoria Lutje, CIDG Information Specialist, for help with the search strategy. Guy Marks, Cecily Miller, and Tamara Kredo contributed important insights into the presentation and analysis of the data as part of the proceedings of the WHO Guideline Development Group for Systematic Screening of Tuberculosis. 


\section{R E F E R E N C E S}

\section{References to studies included in this review}

\section{Al-Darraji 2013 \{published data only\}}

Al-Darraji HA, Abd Razak H, Ng KP, Altice FL, Kamarulzaman A. The diagnostic performance of a single GeneXpert MTB/RIF assay in an intensified tuberculosis case finding survey among HIV-infected prisoners in Malaysia. PLOS One 2013;8(9):e73717.

\section{Al-Darraji 2016 \{published and unpublished data\}}

Al-Darraji HA, Altice FL, Kamarulzaman A. Undiagnosed pulmonary tuberculosis among prisoners in Malaysia: an overlooked risk for tuberculosis in the community. Tropical Medicine and International Health 2016;21(8):1049-58.

Balcha 2014 \{published data only\} Balcha TT, Sturegård E, Winqvist N, Skogmar S, Reepalu A, Jemal ZH, et al. Intensified tuberculosis case-finding in HIVpositive adults managed at Ethiopian health centers: diagnostic yield of Xpert MTB/RIF compared with smear microscopy and liquid culture. PLOS One 2014;9(1):e85478.

\section{Beyanga 2018 \{published data only\}}

Beyanga M, Kidenya BR, Gerwing-Adima L, Ochodo E, Mshana SE, Kasang C. Investigation of household contacts of pulmonary tuberculosis patients increases case detection in Mwanza City, Tanzania. BMC Infectious Diseases 2018;18(1):110.

\section{Bjerrum 2016 \{published data only\}}

Bjerrum S, Oliver-Commey J, Kenu E, Lartey M, Newman MJ, Addo KK, et al. Tuberculosis and non-tuberculous mycobacteria among HIV-infected individuals in Ghana. Tropical Medicine \& International Health 2016;21(9):1181-90.

\section{Dorman 2012 \{published data only\}}

Dorman SE, Chihota VN, Lewis JJ, Shah M, Clark D, Grant AD, et al. Performance characteristics of the Cepheid Xpert MTB/RIF test in a tuberculosis prevalence survey. PLOS One 2012;7(8):e43307.

\section{Heidebrecht 2016 \{published data only\}}

Heidebrecht CL, Podewils LJ, Pym AS, Cohen T, Mthiyane T, Wilson D. Assessing the utility of Xpert $\left({ }^{\oplus}\right)$ MTB/RIF as a screening tool for patients admitted to medical wards in South Africa. Scientific Reports 2016;6:19391.

\section{Henostroza 2016 \{published data only\}}

Henostroza G, Harris JB, Chitambi R, Siyambango M, Turnbull ER, Maggard KR, et al. High prevalence of tuberculosis in newly enrolled HIV patients in Zambia: need for enhanced screening approach. International Journal of Tuberculosis and Lung Disease 2016;20(8):1033-9.

\section{Kempker 2019 \{published data only\}}

Kempker RR, Chkhartishvili N, Kinkladze I, Schechter MC, Harrington K, Rukhadze N, et al. High yield of active tuberculosis case finding among HIV-infected patients using Xpert MTB/RIF testing. Open Forum Infectious Diseases 2019;6(6):ofz233.
LaCourse 2016 \{published data only\}

LaCourse SM, Cranmer LM, Matemo D, Kinuthia J, Richardson BA, John-Stewart G, et al. Tuberculosis case finding in HIV-Infected pregnant women in Kenya reveals poor performance of symptom screening and rapid diagnostic tests. Journal of the Acquired Immune Deficiency Syndrome 2016;71(2):219-27.

\section{Lawn 2011 \{published data only\}}

Lawn SD, Brooks SV, Kranzer K, Nicol MP, Whitelaw A, Vogt M, et al. Screening for HIV-associatd tuberculosis and rifampin resistance before antiretroviral therapy using the Xpert MTB/RIF assay: a prospective study. PLOS Medicine 2011;8(7):e1001067.

Lawn 2012 \{published data only\}

Lawn SD, Kerkhoff AD, Vogt M, Wood R. Diagnostic accuracy of a low-cost, urine antigen, point-of-care screening assay for HIV-associated pulmonary tuberculosis before antiretroviral therapy: a descriptive study. Lancet Infectious Diseases 2012;12(3):201-9.

\section{Lopez-Varela 2019 \{published data only\}}

López-Varela E, Respeito D, Blanco S, Gimo M, Sacoor C, Naniche D, et al. High yield of home-based TB diagnosis among newly diagnosed patients with HIV. Journal of the Acquired Immune Deficiency Syndrome 2019;80(4):e103-e105.

\section{Mollel 2017 \{published data only\}}

Mollel EW, Chilongola JO, Mpagama SG, Kibiki GS. Evaluation of Xpert MTB/Rif performance for diagnosis of tuberculosis among HIV positive patients in northern Tanzania. Tanzania Journal of Health Research 2017;19(1):1-9.

\section{Ntinginya 2012 \{published data only\}}

Ntinginya EN, Squire SB, Millington KA, Mtafya B, Saathoff E, Heinrich N, et al. Performance of the Xpert ${ }^{\circledR}$ MTB/RIF assay in an active case-finding strategy: a pilot study from Tanzania. International Journal of Tuberculosis and Lung Disease 2012;16(11):1468-70.

\section{O'Grady 2012 \{published data only\}}

O'Grady J, Bates M, Chilukutu L, Mzyece J, Cheelo B, Chilufya M, et al. Evaluation of the Xpert MTB/RIF assay at a tertiary care referral hospital in a setting where tuberculosis and HIV infection are highly endemic. Clinical Infectious Diseases 2012;55(9):1171-8.

\section{Reeve 2019a \{published and unpublished data\}}

Reeve B, Ndlangalavu G, Palmer Z, Jackson J, Dolby T, van Helden P, et al. Accuracy of Xpert Ultra and Xpert MTB/ RIF in people living with HIV initiating antiretroviral treatment who have minimal TB symptoms. International Journal of Tuberculosis and Lung Diseases 2019;3(10):S115.

Reeve 2019b \{published and unpublished data\} Reeve B, Ndlangalavu G, Palmer Z, Jackson J, Dolby T, van Helden P, et al. Accuracy of Xpert Ultra and Xpert MTB/ RIF in people living with HIV initiating antiretroviral treatment

Xpert MTB/RIF and Xpert Ultra assays for screening for pulmonary tuberculosis and rifampicin resistance in adults, irrespective of signs 
who have minimal TB symptoms. International Journal of Tuberculosis and Lung Diseases 2019;3(10):S115.

\section{Santos 2020 \{published and unpublished data\}}

Santos AD, Oliveira RD, Lemos EF, Lima F, Cohen T, Cords O, et al. Yield, efficiency and costs of mass screening algorithms for tuberculosis in Brazilian prisons. Clinical Infectious Diseases 2020;72(5):771-7.

\section{Tahseen 2018 \{published data only\}}

Tahseen S, Shahnawaz H, Riaz U, Khanzada FM, Hussain A, Aslam W, et al. Systematic case finding for tuberculosis in HIV-infected people who inject drugs: experience from Pakistan. International Journal of Tuberculosis and Lung Disease 2018;22(2):187-193.

\section{Yoon 2017 \{published data only\}}

Yoon C, Semitala FC, Atuhumuza E, Katende J, Mwebe S, Asege L, et al. Point-of-care C-reactive protein-based tuberculosis screening for people living with HIV: a diagnostic accuracy study. Lancet Infectious Diseases 2017;17(12):1285-92.

\section{References to studies excluded from this review}

\section{Adams 2015 \{published data only\}}

Adams CD, Velez JD, Martinez LF. Xpert((R)) MTB/RIF and very low positive detection in bronchoalveolar lavage: diagnostic concerns. International Journal of Tuberculosis and Lung Disease 2015;19(7):871-3.

\section{Adejumo 2018 \{published data only}

Adejumo OA, Olusola-Faleye B, Adepoju V, Bowale A, Adesola S, Falana A, et al. Prevalence of rifampicin resistant tuberculosis and associated factors among presumptive tuberculosis patients in a secondary referral hospital in Lagos Nigeria. African Health Sciences 2018;18(3):472-8.

\section{Adetunji 2019 \{published data only\}}

Adetunji SO, Donbraye E, Ekong MJ, Adetunji BI. Rifampicinresistant tuberculosis among known HIV-infected patients in Oyo State, Nigeria. Journal of Immunoassay and Immunochemistry 2019;40(3):289-99.

\section{Agizew 2017 \{published data only\}}

Agizew T, Boyd R, Ndwapi N, Auld A, Basotli J, Nyirenda S, et al. Peripheral clinic versus centralized laboratory-based Xpert MTB/RIF performance: experience gained from a pragmatic, stepped-wedge trial in Botswana. PLOS One 2017;12(8):e0183237.

\section{Aia 2016 \{published data only\}}

Aia P, Kal M, Lavu E, John LN, Johnson K, Coulter C, et al. The burden of drug-resistant tuberculosis in Papua New Guinea: results of a large population-based survey. PLOS One 2016;11(3):e0149806.

\section{Antonenka 2013 \{published data only\}}

Antonenka U, Hofmann-Thiel S, Turaev L, Esenalieva A, Abdulloeva M, Sahalchyk E, et al. Comparison of Xpert MTB/ RIF with ProbeTec ET DTB and COBAS TaqMan MTB for direct detection of $\mathrm{M}$ tuberculosis complex in respiratory specimens. BMC Infectious Diseases 2013;13:280.

\section{Ardizzoni 2015 \{published data only\}}

Ardizzoni E, Fajardo E, Saranchuk P, Casenghi M, Page AL, Varaine F, et al. Implementing the Xpert(R) MTB/RIF diagnostic test for tuberculosis and rifampicin resistance: outcomes and lessons learned in 18 countries. PLOS One 2015;10(12):e0144656.

\section{Ardizzoni 2020 \{published data only\}}

Ardizzoni E, Orikiriza P, Ssuuna C, Nyehangane D, Gumsboga M, Taremwa IM. Evaluation of OMNigene sputum and ethanol reagent for preservation of sputum prior to Xpert and culture testing in Uganda. Journal of Clinical Microbiology 2020;58(1):e00810-19.

\section{Assefa 2019 \{published data only\}}

Assefa D, Belachew F, Wondimagegn G, Klinkenberg E. Missed pulmonary tuberculosis: a cross sectional study in the general medical inpatient wards of a large referral hospital in Ethiopia. BMC Infectious Diseases 2019;19(1):60.

\section{Auld 2016a \{published data only\}}

Auld AF, Agizew T, Pals S, Finlay A, Ndwapi N, Boyd R, et al. Implementation of a pragmatic, stepped-wedge cluster randomized trial to evaluate impact of Botswana's Xpert MTB/ RIF diagnostic algorithm on TB diagnostic sensitivity and early antiretroviral therapy mortality. BMC Infectious Diseases 2016;16(1):606.

\section{Auld 2016b \{published data only\}}

Auld SC, Moore BK, Kyle RP, Eng B, Nong K, Pevzner ES, et al. Mixed impact of xpert $^{\circledR}$ MTB/RIF on tuberculosis diagnosis in Cambodia. Public Health Action 2016;6(2):129-35.

\section{Auld 2020 \{published data only\}}

Auld AF, Agizew T, Mathoma A, Boyd R, Date A, Pals SL, et al. Effect of tuberculosis screening and retention interventions on early antiretroviral therapy mortality in Botswana: a steppedwedge cluster randomized trial. BMC Medicine 2020;18(1):19.

\section{Awan 2018 \{published data only\}}

Awan WM, Zaidi SM, Habib SS, Khowaja S, Malik A, Khan U, et al. Impact of scaling up Xpert((R)) MTB/RIF testing for the detection of rifampicin-resistant TB cases in Karachi, Pakistan. International Journal of Tuberculosis and Lung Disease 2018;22(8):899-904.

\section{Ayala 2016 \{published data only\}}

Ayala G, Garay J, Aragon M, Decroo T, Zachariah R. Trends in tuberculosis notification and treatment outcomes in prisons: a country-wide assessment in El Salvador from 2009-2014. Pan American Journal of Public Health (Revista Panamericana de Salud Pública) 2016;39(1):38-43.

\section{Bablishvili 2015 \{published data only\}}

Bablishvili N, Tukvadze N, Avaliani Z, Blumberg HM, Kempker RR. A comparison of the Xpert((R)) MTB/RIF and GenoType((R)) MTBDRplus assays in Georgia. International Journal of Tuberculosis and Lung Disease 2015;19(6):676-8.

Xpert MTB/RIF and Xpert Ultra assays for screening for pulmonary tuberculosis and rifampicin resistance in adults, irrespective of signs 
Bacells 2016 \{published data only\}

Bacells ME, Huilcaman M, Pena C, Castillo C, Carvajal C, Scioscia N, et al. M-tuberculosis DNA detection in nasopharyngeal mucosa can precede tuberculosis development in contacts. International Journal of Tuberculosis and Lung Disease 2016;20(6):848-52.

\section{Balcha 2014a \{published data only\}}

Balcha TT, Winqvist N, Sturegard E, Skogmar S, Reepalu A, Jemal ZH, et al. Detection of lipoarabinomannan in urine for identification of active tuberculosis among HIV-positive adults in Ethiopian health centres. Tropical Medicine \& International Health 2014;19(6):734-42.

\section{Balcha 2015 \{published data only\}}

Balcha TT, Skogmar S, Sturegard E, Bjorkman P, Winqvist N. Outcome of tuberculosis treatment in HIV-positive adults diagnosed through active versus passive case-finding. Global Health Action 2015;8:27048.

\section{Basir 2019 \{published data only\}}

Basir MS, Habib SS, Zaidi SM, Khowaja S, Hussain H, Ferrand RA, et al. Operationalization of bi-directional screening for tuberculosis and diabetes in private sector healthcare clinics in Karachi, Pakistan. BMC Health Services Research 2019;19(1):147.

\section{Bassett 2019 \{published data only\}}

Bassett IV, Forman LS, Govere S, Thulare H, Frank SC, Mhlongo B, et al. Test and Treat TB: a pilot trial of GeneXpert MTB/RIF screening on a mobile HIV testing unit in South Africa. BMC Infectious Diseases 2019;19(1):110.

\section{Benjamin 2019 \{published data only\}}

Benjamin A, Cavalcante SC, Jamal LF, Arakaki-Sanchez D, de Lima JN, Pilotto JH, et al. Accuracy of Determine TB-LAM Ag to detect TB in HIV infected patients associated with diagnostic methods used in Brazilian public health units. PLOS One 2019;14(9):e0221038.

\section{Bhardwaj 2019 \{published data only\}}

Bhardwaj A, Khan S, Kumar A, George L, Mehta A, Radhakrishnan K. Assessing the utility of GeneXpert MTB/ Rif Assay in a tertiary care centre in Southern India with established microscopy and liquid culture facilities. Journal of the Association of Physicians of India 2019;67(8):31-4.

\section{Bjerrum 2015 \{published data only\}}

Bjerrum S, Kenu E, Lartey M, Newman MJ, Addo KK, Andersen $A B$, et al. Diagnostic accuracy of the rapid urine lipoarabinomannan test for pulmonary tuberculosis among HIV-infected adults in Ghana-findings from the DETECT HIV-TB study. BMC Infectious Diseases 2015;15:407.

\section{Blakemore 2011 \{published data only\}}

Blakemore R, Nabeta P, Davidow AL, Vadwai V, Tahirli R, Munsamy $\mathrm{V}$, et al. A multisite assessment of the quantitative capabilities of the Xpert MTB/RIF assay. American Journal of Respiratory and Critical Care Medicine 2011;184(9):1076-84.

\section{Boum 2016 \{published data only}

Boum Y 2nd, Kim S, Orikiriza P, Acuna-Villaorduna C, Vinhas S, Bonnet $\mathrm{M}$, et al. Diagnostic accuracy of the small membrane filtration method for diagnosis of pulmonary tuberculosis in a high-HIV-prevalence setting. Journal of Clinical Microbiology 2016;54(6):1520-7.

\section{Byashalira 2019 \{published data only\}}

Byashalira K, Mbelele P, Semvua H, Chilongola J, Semvua S, Liyoyo A, et al. Clinical outcomes of new algorithm for diagnosis and treatment of tuberculosis sepsis in HIV patients. International Journal of Mycobacteriology 2019;8(4):313-9.

\section{Calligaro 2017 \{published data only\}}

Calligaro GL, Zijenah LS, Peter JG, Theron G, Buser V, McNerney R, et al. Effect of new tuberculosis diagnostic technologies on community-based intensified case finding: a multicentre randomised controlled trial. Lancet Infectious Diseases 2017;17(4):441-50.

\section{Carmone 2017 \{published data only\}}

Carmone A, Rodriguez CA, Frank TD, Kiromat M, Bongi PW, Kuno RG, et al. Increasing isoniazid preventive therapy uptake in an HIV program in rural Papua New Guinea. Public Health Action 2017;7(3):193-8.

\section{Cavanaugh 2016 \{published data only\}}

Cavanaugh JS, Modi S, Musau S, McCarthy K, Alexander H, Burmen B, et al. Comparative yield of different diagnostic tests for tuberculosis among people living with HIV in Western Kenya. PLOS One 2016;11(3):e0152364.

\section{Celik 2015 \{published data only\}}

Celik C, Gozel MG, Bakici MZ, Berk S, Ozsahin SL, Gulturk E. Applicability of Xpert MTB/RIF assay for routine diagnosis of tuberculosis: a four-year single-center experience. Turkish Journal of Medical Sciences 2015;45(6):1329-34.

\section{Charoensook 2018 \{published data only\}}

Charoensook P, Upala P, Anuwatnonthakate A, Ruanjai T, Apidechkul T. Pulmonary tuberculosis screening and quality of life among migrant workers, Northern Thailand. Journal of Infection in Developing Countries 2018;12(12):1052-61.

\section{Chry 2020 \{published data only\}}

Chry M, Smelyanskaya M, Ky M, Codlin AJ, Cazabon D, Eang MT, et al. Can the high sensitivity of Xpert MTB/RIF Ultra be harnessed to save cartridge costs? results from a pooled sputum evaluation in Cambodia. Tropical Medicine and Infectious Disease 2020;5(1):27.

\section{Chumpa 2020 \{published data only\}}

Chumpa N, Kawkitinarong K, Rotcheewaphan S, Sawatpanich A, Petsong S, Tumwasorn S, et al. Evaluation of AnyplexTM II MTB/MDR kit's performance to rapidly detect isoniazid and rifampicin resistant Mycobacterium tuberculosis from various clinical specimens. Molecular Biology Reports 2020;47(4):2501-8.

Deshmukh 2020 \{published data only\}

Deshmukh S, Atre S, Chavan A, Raskar S, Sawant T, Mave V, et al. Assessment of the Xpert assay among adult pulmonary

Xpert MTB/RIF and Xpert Ultra assays for screening for pulmonary tuberculosis and rifampicin resistance in adults, irrespective of signs 
tuberculosis suspects with and without diabetes mellitus. International Union against Tuberculosis and Lung Disease 2020;24(1):113-7.

\section{Ekeke 2020 \{published data only\}}

Ekeke N, Aniwada E, Chukwu J, Nwafor C, Meka A, Chukwuka A, et al. Screening diabetes mellitus patients for tuberculosis in Southern Nigeria: a pilot study. Advances in Respiratory Medicine 2020;88(1):6-12.

\section{Farra 2017 \{published data only\}}

Farra A, Manirakiza A, Yambiyo BM, Zandanga G, Lokoti B, Berlioz-Arthaud A, et al. Surveillance of rifampicin resistance with GeneXpert MTB/RIF in the National Reference Laboratory for tuberculosis at the Institut Pasteur in Bangui, 2015-2017. Open Forum Infectious Diseases 2019;6(3):ofz075.

\section{Floridia 2017 \{published data only\}}

Floridia M, Ciccacci F, Andreotti M, Hassane A, Sidumo Z, Magid NA, et al. Tuberculosis case finding with combined rapid point-of-care assays (Xpert MTB/RIF and Determine TB LAM) in HIV-positive individuals starting antiretroviral therapy in Mozambique. Clinical Infectious Diseases 2017;65(11):1878-83.

\section{Gautam 2019 \{published data only\}}

Gautam H, Singla M, Jain R, Lodha R, Kabra SK, Singh UB. Point-of-care urine lipoarabinomannan antigen detection for diagnosis of tuberculosis in children. International Journal of Tuberculosis and Lung Disease 2019;23(6):714-9.

\section{Gelalcha 2017 \{published data only\}}

Gelalcha AG, Kebede A, Mamo H. Light-emitting diode fluorescent microscopy and Xpert MTB/RIF(R) assay for diagnosis of pulmonary tuberculosis among patients attending Ambo hospital, west-central Ethiopia. BMC Infectious Diseases 2017;17(1):613.

\section{Gizachew 2017 \{published data only\}}

Gizachew Beza M, Hunegnaw E, Tiruneh M. Prevalence and associated factors of tuberculosis in prisons settings of East Gojjam Zone, Northwest Ethiopia. International Journal of Bacteriology 2017;2017:3826980.

\section{Gupta-Wright 2018 \{published data only\}}

Gupta-Wright A, Corbett EL, van Oosterhout JJ, Wilson D, Grint D, Alufandika-Moyo M, et al. Rapid urine-based screening for tuberculosis in HIV-positive patients admitted to hospital in Africa (STAMP): a pragmatic, multicentre, parallelgroup, double-blind, randomised controlled trial. Lancet 2018;392(10144):292-301.

\section{Gursoy 2016 \{published data only\}}

Gursoy NC, Yakupogullari Y, Tekerekoglu MS, Otlu B. [Evaluation of the diagnostic performance of Xpert MTB/RIF test for the detection of Mycobacterium tuberculosis and rifampin resistance in clinical samples]. Mikrobiyoloji Bulteni 2016;50(2):196-204.

\section{Habeenzu 2017 \{published data only\}}

Habeenzu C, Nakajima C, Solo E, Bwalya P, Kajino K, Miller M, et al. Evaluation of in-house loop-mediated isothermal amplification for tuberculosis diagnosis compared with Xpert MTB/RIF. Journal of Infection in Developing Countries 2017;11(6):440-4.

\section{Habte 2016 \{published data only\}}

Habte D, Melese M, Hiruy N, Gashu Z, Jerene D, Moges F, et al. The additional yield of GeneXpert MTB/RIF test in the diagnosis of pulmonary tuberculosis among household contacts of smear positive TB cases. International Journal of Infectious Diseases 2016;49:179-84.

\section{Hanifa 2016 \{published data only\}}

Hanifa Y, Fielding KL, Chihota VN, Adonis L, Charalambous S, Karstaedt A, et al. Diagnostic accuracy of lateral flow urine LAM assay for TB screening of adults with advanced immunosuppression attending routine HIV care in South Africa. PLOS One 2016;11(6):e0156866.

\section{Head 2019 \{published data only\}}

Head IM, Lazarus R. Use of Xpert MTB/RIF in a low prevalence setting in the Southwest of England. Journal of Infection 2019;S0163-4453(19):30345-7.

\section{Hiruy 2018 \{published data only\}}

Hiruy N, Melese M, Habte D, Jerene D, Gashu Z, Alem G, et al. Comparison of the yield of tuberculosis among contacts of multidrug-resistant and drug-sensitive tuberculosis patients in Ethiopia using GeneXpert as a primary diagnostic test. International Journal of Infectious Diseases 2018;71:4-8.

\section{Ho 2016 \{published data only\}}

Ho J, Nguyen PT, Nguyen TA, Tran KH, Van Nguyen S, Nguyen NV, et al. Reassessment of the positive predictive value and specificity of Xpert MTB/RIF: a diagnostic accuracy study in the context of community-wide screening for tuberculosis. Lancet Infectious Diseases 2016;16(9):1045-51.

\section{Hosseinipour 2016 \{published data only\}}

Hosseinipour MC, Bisson GP, Miyahara S, Sun X, Moses A, Riviere $C$, et al. Empirical tuberculosis therapy versus isoniazid in adult outpatients with advanced HIV initiating antiretroviral therapy (REMEMBER): a multicountry open-label randomised controlled trial. Lancet 2016;387(10024):1198-209.

\section{Huang 2018 \{published data only\}}

Huang $\mathrm{H}$, Zhang Y, Li S, Wang J, Chen J, Pan Z, et al. Rifampicin resistance and multidrug-resistant tuberculosis detection using Xpert MTB/RIF in Wuhan, China: a retrospective study. Microbial Drug Resistance 2018;24(5):675-9.

\section{Huerga 2020 \{published data only\}}

Huerga H, Cossa L, Manhiça I, Bastard M, Telnov A, Molfino L, et al. Systematic, point-of-care urine lipoarabinomannan (Alere TB-LAM) assay for diagnosing tuberculosis in severely immunocompromised HIV-Positive ambulatory patients. American Journal of Tropical Medicine and Hygiene 2020;102(3):562-6

\section{Huh 2019 \{published data only\}}

Huh HJ, Song DJ, Ki CS, Lee NY. Is cross-reactivity with nontuberculous mycobacteria a systematic problem in the

Xpert MTB/RIF and Xpert Ultra assays for screening for pulmonary tuberculosis and rifampicin resistance in adults, irrespective of signs 
Xpert MTB/RIF assay? Tuberculosis and Respiratory Diseases 2019;82(1):88-9.

\section{Kamenska 2019 \{published data only\}}

Kamenska N, Nabirova D, Davtyan K, Davtyan H, Zachariah R, Aslanyan G. Strategies for active detection of tuberculosis in Ukraine: Comparative effectiveness amongst key populations (2014-2018). Journal of Infection in Developing Countries 2019;13(7.1):89s-94s.

\section{Kerkhoff 2014 \{published data only\}}

Kerkhoff AD, Wood R, Vogt M, Lawn SD. Prognostic value of a quantitative analysis of lipoarabinomannan in urine from patients with HIV-associated tuberculosis. PLOS One 2014;9(7):e103285.

\section{Kurbaniyazova 2017 \{published data only\}}

Kurbaniyazova G, Joncevska M, Kalon S, Kalmambetova G, Mohr T, Toktogonova A, et al. Results of Xpert MTB/RIF implementation in Kyrgyzstan. International Journal of Tuberculosis and Lung Disease 2017;21(3):333-7.

\section{Kuyinu 2018 \{published data only\}}

Kuyinu YA, Odugbemi BA, Salisu-Olatunji SO, Adepoju FO, Odusanya OO. Characteristics of Mycobacterium tuberculosis positive patients screened for drug-resistant tuberculosis at a tertiary health facility in Lagos, Nigeria. Journal of the National Medical Association 2018;110(1):88-91.

\section{LaCourse 2014 \{published data only\}}

LaCourse SM, Chester FM, Preidis G, McCrary LM, ArscottMills T, Maliwichi M, et al. Use of Xpert for the diagnosis of pulmonary tuberculosis in severely malnourished hospitalized Malawian children. Pediatric Infectious Disease Journal 2014;33(11):1200-2.

\section{LaCourse 2018 \{published data only\}}

LaCourse SM, Pavlinac PB, Cranmer LM, Njuguna IN, Mugo C, Gatimu J, et al. Stool Xpert MTB/RIF and urine lipoarabinomannan for the diagnosis of tuberculosis in hospitalized HIV-infected children. AIDS 2018;32(1):69-78.

\section{Lawn 2012a \{published data only\}}

Lawn SD, Kerkhoff AD, Vogt M, Ghebrekristos Y, Whitelaw A, Wood R. Characteristics and early outcomes of patients with Xpert MTB/RIF-negative pulmonary tuberculosis diagnosed during screening before antiretroviral therapy. Clinical Infectious Diseases 2012;54(8):1071-9.

\section{Lawn 2012b \{published data only\}}

Lawn SD, Kerkhoff AD, Vogt M, Wood R. Clinical significance of lipoarabinomannan detection in urine using a low-cost pointof-care diagnostic assay for HIV-associated tuberculosis. AIDS 2012;26(13):1635-43.

\section{Lawn 2013 \{published data only\}}

Lawn SD, Kerkhoff AD, Vogt M, Wood R. HIV-associated tuberculosis: relationship between disease severity and the sensitivity of new sputum-based and urine-based diagnostic assays. BMC Medicine 2013;11:231.

\section{Lawn 2015 \{published data only\}}

Lawn SD, Kerkhoff AD, Burton R, Schutz C, van Wyk G, Vogt M, et al. Rapid microbiological screening for tuberculosis in HIVpositive patients on the first day of acute hospital admission by systematic testing of urine samples using Xpert MTB/RIF: a prospective cohort in South Africa. BMC Medicine 2015;13:192.

\section{Lawn 2017 \{published data only\}}

Lawn SD, Kerkhoff AD, Burton R, Schutz C, Boulle A, Vogt M, et al. Diagnostic accuracy, incremental yield and prognostic value of Determine TB-LAM for routine diagnostic testing for tuberculosis in HIV-infected patients requiring acute hospital admission in South Africa: a prospective cohort. BMC Medicine 2017;15(1):67.

\section{Lebina 2016 \{published data only\}}

Lebina L, Fuller N, Osoba T, Scott L, Motlhaoleng K, Rakgokong M, et al. The Use of Xpert MTB/Rif for active case finding among TB contacts in North West Province, South Africa. Tuberculosis Research and Treatment 2016;2016:4282313.

\section{Lima 2020 \{published data only\}}

Lima F, Santos AS, Oliveira RD, Silva CC, Goncalves CC, Andrews JR, et al. Oral swab testing by Xpert(R) MTB/RIF Ultra for mass tuberculosis screening in prisons. Journal of Clinical Tuberculosis and Other Mycobacterial Diseases 2020;19:100148.

\section{Luo 2019 \{published data only\}}

Luo J, Luo M, Li J, Yu J, Yang H, Yi X, et al. Rapid direct drug susceptibility testing of Mycobacterium tuberculosis based on culture droplet digital polymerase chain reaction. International Journal of Tuberculosis and Lung Disease 2019;23(2):219-25.

\section{Maria 2018 \{published data only\}}

Maria MC, Rosarys MR, Misleidis SA, Grechen GL, Secretario CT, Raul DR. Diagnostic importance of "GeneXpert Mtb-Rif" in patients infected by the human immunodeficiency virus (HIV) [Spanish]. Archivos Venezolanos de Farmacologia y Terapeutica 2018;37(4):355-8

\section{Marks 2019 \{published data only\}}

Marks GB, Nguyen NV, Nguyen PT, Nguyen TA, Nguyen HB, Tran $\mathrm{KH}$, et al. Community-wide screening for tuberculosis in a high-prevalence setting. New England Journal of Medicine 2019;381(14):1347-57.

\section{Marlowe 2011 \{published data only\}}

Marlowe EM, Novak-Weekley SM, Cumpio J, Sharp SE, Momeny MA, Babst A, et al. Evaluation of the Cepheid Xpert MTB/RIF assay for direct detection of Mycobacterium tuberculosis complex in respiratory specimens. Journal of Clinical Microbiology 2011;49(4):1621-3.

\section{Mbatchou 2019 \{published data only\}}

Mbatchou Ngahane B, Gaping Simen S, Halle M, Okalla C, Goupeyou Wandji IA. Prevalence of tuberculosis and its factors among patients on maintenance dialysis in Douala, Cameroon. American Journal of Respiratory and Critical Care Medicine. Conference 2019;201(Meeting abstracts):A5146.

Xpert MTB/RIF and Xpert Ultra assays for screening for pulmonary tuberculosis and rifampicin resistance in adults, irrespective of signs 


\section{Mbu 2018 \{published data only\}}

Mbu ET, Sauter F, Zoufaly A, Bronsvoort BM, Morgan KL, Noeske J, et al. Tuberculosis in people newly diagnosed with HIV at a large HIV care and treatment center in Northwest Cameroon: Burden, comparative screening and diagnostic yields, and patient outcomes. PLOS One 2018;13(6):e0199634.

\section{Meng 2017 \{published data only\}}

Meng C, Shen Y, Wang J, Wang S, Chen X, Yu S, et al. A two-step algorithm for rapid diagnosis of active pulmonary tuberculosis in entry applicants using the T-SPOT.TB and Xpert MTB/RIF assays in Shanghai, China. Emerging Microbes \& Infections 2017;6(7):e67.

\section{Metcalfe 2015 \{published data only\}}

Metcalfe JZ, Makumbirofa S, Makamure B, Mutetwa R, Penaloza RA, Sandy C, et al. Suboptimal specificity of Xpert MTB/RIF among treatment-experienced patients. European Respiratory Journal 2015;45(5):1504-6.

\section{Metcalfe 2016 \{published data only\}}

Metcalfe JZ, Makumbirofa S, Makamure B, Sandy C, Bara W, Mason P, et al. Xpert((R)) MTB/RIF detection of rifampin resistance and time to treatment initiation in Harare, Zimbabwe. International Journal of Tuberculosis and Lung Disease 2016;20(7):882-9.

\section{Miller 2011 \{published data only\}}

Miller MB, Popowitch EB, Backlund MG, Ager EP. Performance of Xpert MTB/RIF RUO assay and IS6110 real-time PCR for Mycobacterium tuberculosis detection in clinical samples. Journal of Clinical Microbiology 2011;49(10):3458-62.

\section{Mishra 2020 \{published data only\}}

Mishra H, Reeve BW, Palmer Z, Caldwell J, Dolby T, Naidoo CC. Xpert MTB/RIF Ultra and Xpert MTB/RIF for diagnosis of tuberculosis in an HIV-endemic setting with a high burden of previous tuberculosis: a two-cohort diagnostic accuracy study. Lancet Respiratory Medicine 2020;8(4):368-82.

\section{Modi 2016 \{published data only\}}

Modi S, Cavanaugh JS, Shiraishi RW, Alexander HL, McCarthy KD, Burmen B, et al. Performance of clinical screening algorithms for tuberculosis intensified case finding among people living with HIV in Western Kenya. PLOS One 2016;11(12):e0167685.

\section{Morishita 2017 \{published data only\}}

Morishita F, Garfin AM, Lew W, Oh KH, Yadav RP, Reston JC, et al. Bringing state-of-the-art diagnostics to vulnerable populations: the use of a mobile screening unit in active case finding for tuberculosis in Palawan, the Philippines. PLOS One 2017;12(2):e0171310.

\section{Nathavitharana 2017 \{published data only\}}

Nathavitharana RR, Daru P, Barrera AE, Kamal SM, Islam S, Ul-Alam M, et al. FAST implementation in Bangladesh: High frequency of unsuspected tuberculosis justifies challenges of scale-up. International Journal of Tuberculosis and Lung Disease 2017;21(9):1020-5.

\section{Nicol 2018 \{published data only\}}

Nicol MP, Workman L, Prins M, Bateman L, Ghebrekristos Y, Mbhele S, et al. Accuracy of Xpert MTB/RIF Ultra for the diagnosis of pulmonary tuberculosis in children. Pediatric Infectious Disease Journal 2018;37(10):e261-e3.

\section{Nikolayevskyy 2019 \{published data only\}}

Nikolayevskyy V, Kontsevaya I, Nikolaevskaya E, Surkova E, Samchenko S, Esipenko S. Diagnostic performance and impact of routinely implemented Xpert(R) MTB/RIF assay in a setting of high incidence of drug-resistant TB in Odessa Oblast, Ukraine. In: Clinical Microbiology and Infection. Vol. 25. 2019:1040.e1-1040.e6.

\section{Ou 2019 \{published data only\}}

Ou XC, Li H, Liu DX, Xia H, Ma XG, Wang SH, et al. Comparison of Xpert MTB/RIF, RealAmp, and CPA tests in detecting Mycobacterium tuberculosis. Biomedical and Environmental Sciences 2019;32(3):215-9.

\section{Ozkutuk 2014 \{published data only\}}

Ozkutuk N, Surucuoglu S. [Evaluation of the Xpert MTB/RIF assay for the diagnosis of pulmonary and extrapulmonary tuberculosis in an intermediate-prevalence setting]. Mikrobiyoloji Bulteni 2014;48(2):223-32.

\section{Parcell 2017 \{published data only\}}

Parcell BJ, Jarchow-MacDonald AA, Seagar AL, Laurenson IF, Prescott GJ, Lockhart M. Three year evaluation of Xpert MTB/RIF in a low prevalence tuberculosis setting: a Scottish perspective. Journal of Infection 2017;74(5):466-72.

\section{Park 2013 \{published data only\}}

Park KS, Kim JY, Lee JW, Hwang YY, Jeon K, Koh WJ, et al. Comparison of the Xpert MTB/RIF and Cobas TaqMan MTB assays for detection of Mycobacterium tuberculosis in respiratory specimens. Journal of Clinical Microbiology 2013;51(10):3225-7.

\section{Pimkina 2015 \{published data only\}}

Pimkina E, Zablockis R, Nikolayevskyy V, Danila E, Davidaviciene $E$. The Xpert(R) MTB/RIF assay in routine diagnosis of pulmonary tuberculosis: a multicentre study in Lithuania. Respiratory Medicine 2015;109(11):1484-9.

\section{Ramamurthy 2016 \{published data only\}}

Ramamurthy K, Bhat S, Shenoy S, Rangnekar A. Xpert Mycobacterium tuberculosis/rifampicin assay: a boon in tuberculosis diagnostics. Asian Journal of Pharmaceutical and Clinical Research 2016;9(5):225-7.

\section{Reepalu 2016 \{published data only\}}

Reepalu A, Balcha TT, Skogmar S, Güner N, Sturegård E, Björkman P. Factors associated with early mortality in HIVpositive men and women investigated for tuberculosis at Ethiopian health centers. PLOS One 2016;11(6):e0156602.

\section{Reis 2019 \{published data only\}}

Reis AJ, Diniz JL, Silva AB, Silveira J, Basso R, Vieira R, et al. Laboratory tools for tuberculosis control in a setting with

Xpert MTB/RIF and Xpert Ultra assays for screening for pulmonary tuberculosis and rifampicin resistance in adults, irrespective of signs 
a high burden of HIV/AIDS. Journal of Medical Microbiology 2019;68(11):1622-8.

\section{Sarinoglu 2020 \{published data only\}}

Sarinoglu RC, Duman N, Unlu N, Yildizeli SO, Yagci AK. Xpert MTB/Ultra assay: handle with care. Journal of Infection 2020;80(3):350-71.

\section{Semitala 2019 \{published data only\}}

Semitala FC, Cattamanchi A, Andama A, Atuhumuza E, Katende J, Mwebe S, et al. Brief report: yield and efficiency of intensified tuberculosis case-finding algorithms in 2 highrisk HIV subgroups in Uganda. Journal of Acquired Immune Deficiency Syndromes 2019;82(4):416-20.

\section{Shah 2019 \{published data only\}}

Shah I, Bhamre R, Shetty NS. Accuracy of Xpert Mycobacterium tuberculosis/rifampicin assay in diagnosis of pulmonary tuberculosis. Infectious Diseases 2019;51(7):550-3.

\section{Sun 2019 \{published data only\}}

Sun DF, Zheng LL, Jin F, Wang MS. Evaluation of the double sputum Xpert tests for the diagnosis of pulmonary tuberculosis. Infectious Diseases 2019;51(7):541-2.

\section{Teo 2011 \{published data only\}}

Teo J, Jureen R, Chiang D, Chan D, Lin R. Comparison of two nucleic acid amplification assays, the Xpert MTB/RIF assay and the amplified Mycobacterium tuberculosis direct assay, for detection of Mycobacterium tuberculosis in respiratory and nonrespiratory specimens. Journal of Clinical Microbiology 2011;49(10):3659-62.

\section{Trajman 2014 \{published data only\}}

Trajman A, Durovni B, Saraceni V, Cordeiro-Santos M, Cobelens F, van den Hof S. High positive predictive value of Xpert in a low rifampicin resistance prevalence setting. European Respiratory Journal 2014;44(6):1711-3.

\section{van Kampen 2015 \{published data only\}}

van Kampen SC, Tursynbayeva A, Koptleuova A, Murzakhmetova Z, Bigalieva L, Aubakirova M, et al. Effect of introducing Xpert MTB/RIF to test and treat individuals at risk of multidrug-resistant tuberculosis in Kazakhstan: a prospective cohort study. PLOS One 2015;10(7):e0132514.

Van Rie 2011 \{published data only\}

Van Rie A. A single Xpert MTB/RIF test of sputum for diagnosis of tuberculosis and multidrug resistance shows high sensitivity and specificity and reduces diagnosis and treatment delays. Evidence-Based Medicine 2011;16(6):174-5.

\section{Yasemin 2019 \{published data only\}}

Yasemin A, Ahmad S, Afzal S, Ullah A, Sheed A. Evaluation of GeneXpert MTB/RIF assay for detection of pulmonary tuberculosis on sputum samples. Journal of College of Physicians and Surgeons Pakistan 2019;29(1):66-9.

Yoon 2019 \{published data only\} Yoon C, Semitala FC, Asege L, Katende J, Mwebe S, Andama AO, et al. Yield and efficiency of novel intensified tuberculosis case- finding algorithms for people living with HIV. American Journal of Respiratory and Critical Care Medicine 2019;199(5):643-50.

\section{Additional references}

\section{Balshem 2011}

Balshem H, Helfand M, Schünemann HJ, Oxman AD, Kunz R, Brozek J, et al. GRADE guidelines: 3. Rating the quality of evidence. Journal of Clinical Epidemiology 2011;64(4):401-6.

\section{Banada 2010}

Banada PP, Sivasubramani SK, Blakemore R, Boehme C, Perkins MD, Fennelly K, et al. Containment of bioaerosol infection risk by the Xpert MTB/RIF assay and its applicability to point-of-care settings. Journal of Clinical Microbiology 2010;48(10):3551-7.

\section{Bjerrum 2019}

Bjerrum S, Schiller I, Dendukuri N, Kohli M, Nathavitharana RR, Zwerling AA, et al. Lateral flow urine lipoarabinomannan assay for detecting active tuberculosis in people living with HIV. Cochrane Database of Systematic Reviews 2019, Issue 10. Art. No: CD011420. [DOI: 10.1002/14651858.CD011420.pub3]

\section{Broger 2020}

Broger T, Nicol MP, Székely R, Bjerrum S, Sossen B, Schutz C, et al. Diagnostic accuracy of a novel tuberculosis point-of-care urine lipoarabinomannan assay for people living with HIV: a meta-analysis of individual in- and outpatient data. PLOS Medicine 2020;17(5):e1003113.

\section{Cain 2010}

Cain KP, McCarthy KD, Heilig CM, Monkongdee P, Tasaneeyapan T, Kanara N, et al. An algorithm for tuberculosis screening and diagnosis in people with HIV. New England Journal of Medicine 2010;362:707-16.

\section{Cepheid 2018}

Cepheid. Brochure: Xpert ${ }^{\circledR}$ MTB/RIF Ultra. https:// www.cepheid.com/en/tests/Critical-Infectious-Diseases/XpertMTB-RIF-Ultra (accessed 21 January 2021).

\section{Cepheid 2019}

Cepheid. Brochure: Xpert ${ }^{\circledR}$ MTB/RIF assay. Xpert-MTB-RIFENGLISH-Package-Insert-301-1404-Rev-F.pdf (accessed 20 January 2021).

\section{Chakravorty 2017}

Chakravorty S, Simmons AM, Rowneki M, Parmar H, Cao Y, Ryan J, et al. The new Xpert MTB/RIF Ultra: improving detection of Mycobacterium tuberculosis and resistance to rifampin in an assay suitable for point-of-care testing. mBio 2017;8(4):1-12.

\section{Chu 2006}

Chu H, Cole SR. Bivariate meta-analysis of sensitivity and specificity with sparse data: a generalized linear mixed model approach. Journal of Clinical Epidemiology 2006;59(12):1331-2.

Xpert MTB/RIF and Xpert Ultra assays for screening for pulmonary tuberculosis and rifampicin resistance in adults, irrespective of signs 


\section{Cochrane Special Collection 2020}

Cochrane Infectious Diseases Group. Diagnosing tuberculosis. www.cochranelibrary.com/collections/doi/SC000034/full (accessed 20 January 2021).

\section{Covidence [Computer program]}

Veritas Health Innovation Covidence systematic review software. Available at www.covidence.org. Melbourne, Australia: Veritas Health Innovation, (accessed 10 September 2020). Available at covidence.org.

\section{Dorman 2018}

Dorman SE, Schumacher SG, Alland D, Nabeta P, Armstrong DT, King B, et al. Xpert MTB/RIF Ultra for detection of Mycobacterium tuberculosis and rifampicin resistance: a prospective multicentre diagnostic accuracy study. Lancet Infectious Diseases 2018;18(1):76-84.

\section{Enos 2018}

Enos M, Sitienei J, Ong'ang'o J, Mungai B, Kamene M, Wambugu J, et al. Kenya tuberculosis prevalence survey 2016: Challenges and opportunities of ending TB in Kenya. PLOS One 2018;13(12):e0209098.

\section{Ford 2016}

Ford N, Matteelli A, Shubber Z, Hermans S, Meintjes G, Grinsztejn B, et al. TB as a cause of hospitalization and inhospital mortality among people living with HIV worldwide: a systematic review and meta-analysis. Journal of the International AIDS Society 2016;19(1):20714.

\section{Frascella 2020}

Frascella B, Richards AS, Sossen B, Emery JC, Odone A, Law I, et al. Subclinical tuberculosis disease - a review and analysis of prevalence surveys to inform definitions, burden, associations, and screening methodology. Clinical Infectious Diseases 2020 Sept 16 [Epub ahead of print]:ciaa1402. [DOI: 10.1093/cid/ ciaa1402]

\section{Getahun 2010}

Getahun H, Gunneberg C, Granich R, Nunn P. HIV infectionassociated tuberculosis: the epidemiology and the response. Clinical Infectious Diseases 2010;50 Suppl 3:S201-7.

\section{Global Laboratory Initiative 2019}

Global Laboratory Intiaitive. Practical guide to implementing a quality assurance system for Xpert MTB/RIF testing. www.stoptb.org/wg/gli/assets/documents/Xpert-QAguide-2019.pdf (accessed 7 January 2020).

\section{GRADEpro GDT 2020 [Computer program]}

McMaster University (developed by Evidence Prime) GRADEpro GDT. Version accessed after 12 June 2020. Hamilton (ON): McMaster University (developed by Evidence Prime), 2020. Available at gradepro.org.

\section{Gunasekera 2020}

Gunasekera K, Cohen T, Gao W, Ayles H, Godfrey-Faussett P, Claessens M. Smoking and HIV associated with subclinical tuberculosis: analysis of a population-based prevalence survey. International Journal of Tuberculosis and Lung Disease 2020;24(3):340-346

\section{Hamada 2018}

Hamada Y, Lujan J, Schenkel K, Ford N, Getahun H. Sensitivity and specificity of WHO's recommended four-symptom screening rule for tuberculosis in people living with HIV: a systematic review and meta-analysis. Lancet HIV 2018;5(9):e515-23.

\section{Harris 2019}

Harris M, Qi A, Jeagal L, Torabi N, Menzies D, Korobitsyn A, et al. A systematic review of the diagnostic accuracy of artificial intelligence-based computer programs to analyze chest X-rays for pulmonary tuberculosis. PLOS One 2019;14(9):e0221339.

\section{Horne 2019}

Horne DJ, Kohli M, Zifodya JS, Schiller I, Dendukuri N, Tollefson D, et al. Xpert MTB/RIF and Xpert MTB/RIF Ultra for pulmonary tuberculosis and rifampicin resistance in adults. Cochrane Database of Systematic Reviews 2019, Issue 6. Art. No: CD009593. [DOI: 10.1002/14651858.CD009593.pub4]

\section{Lewinsohn 2017}

Lewinsohn DM, Leonard MK, LoBue PA, Cohn DL, Daley CL, Desmond E, et al. Official American Thoracic Society/Infectious Diseases Society of America/Centers for Disease Control and Prevention Clinical Practice Guidelines: diagnosis of tuberculosis in adults and children. Clinical Infectious Diseases 2017;64(2):e1-e33. [PMID: 27932390]

\section{Lunn 2009}

Lunn D, Spiegelhalter D, Thomas A, Best N. The BUGS project: evolution, critique, and future directions. Statistics in Medicine 2009;28(25):3049-67.

\section{Macaskill 2010}

Macaskill P, Gatsonis C, Deeks JJ, Harbord RM, Takwoingi Y. Chapter 10: Analysing and presenting results. In: Deeks JJ, Bossuyt PM, Gatsonis C, editors, Cochrane Handbook for Systematic Reviews of Diagnostic Test Accuracy Version 1.0. The Cochrane Collaboration, 2010. Available from srdta.cochrane.org.

\section{Moher 2009}

Moher D, Liberati A, Tetzlaff J, Altman DG, The PRISMA Group (2009). Preferred reporting items for systematic reviews and meta-analyses: the PRISMA Statement. PLOS Medicine 6;7:e1000097. [DOI: 10.1371/journal.pmed1000097]

\section{Nguyen 2020}

Nguyen HV, Tiemersma EW, Nguyen HB, Cobelens FG, Finlay A, Glaziou P, et al. The second national tuberculosis prevalence survey in Vietnam. PLOS One 2020;15(4):e0232142.

\section{Peter 2016}

Peter JG, Zijenah LS, Chanda D, Clowes P, Lesosky M, Gina P, et al. Effect on mortality of point-of-care, urine-based lipoarabinomannan testing to guide tuberculosis treatment initiation in HIV-positive hospital inpatients: a pragmatic,

Xpert MTB/RIF and Xpert Ultra assays for screening for pulmonary tuberculosis and rifampicin resistance in adults, irrespective of signs 
parallel-group, multicountry, open-label, randomised controlled trial. Lancet 2016;387(10024):1187-97.

\section{R Core Team 2019 [Computer program]}

R Foundation for Statistical Computing R Core Team (2019). R: A language and environment for statistical computing. Version accessed after 12 June 2020. Vienna, Austria: R Foundation for Statistical Computing, 2019. Available at www.R-project.org.

\section{Reitsma 2005}

Reitsma JB, Glas AS, Rutjes AW, Scholten RJ, Bossuyt PM, Zwinderman AH. Bivariate analysis of sensitivity and specificity produces informative summary measures in diagnostic reviews. Journal of Clinical Epidemiology 2005;58(10):982-90.

\section{Review Manager 2020 [Computer program]}

Nordic Cochrane Centre, The Cochrane Collaboration Review Manager 5 (RevMan 5). Version 5.4. Copenhagen: Nordic Cochrane Centre, The Cochrane Collaboration, 2020.

\section{Schünemann 2008}

Schünemann HJ, Oxman AD, Brozek J, Glasziou P, Jaeschke R, Vist GE, et al. Grading quality of evidence and strength of recommendations for diagnostic tests and strategies. BMJ 2008;336(7653):1106-10.

\section{Schünemann 2016}

Schünemann HJ, Mustafa R, Brozek J, Santesso N, AlonsoCoello P, Guyatt G, et al. GRADE Working Group. GRADE Guidelines: 16. GRADE evidence to decision frameworks for tests in clinical practice and public health. Journal of Clinical Epidemiology 2016;76:89-98. [DOI: 10.1016/ j.jclinepi.2016.01.032]

\section{Schünemann 2020a}

Schünemann HJ, Mustafa R, Brozek J, Steingart KR, Leeflang M, Murad MH, et al. GRADE guidelines: 21 part 1 . Study design, risk of bias and indirectness in rating the certainty across a body of evidence for test accuracy. Journal of Clinical Epidemiology 2020;122:129-41. [DOI: 10.1016/j.jclinepi.2019.12.020]

\section{Schünemann 2020b}

Schünemann HJ, Mustafa R, Brozek J, Steingart KR, Leeflang M, Murad MH, et al. GRADE guidelines: 21 part 2. Inconsistency, Imprecision, publication bias and other domains for rating the certainty of evidence for test accuracy and presenting it in evidence profiles and summary of findings tables. Journal of Clinical Epidemiology 2020;122:142-52. [DOI: 10.1016/ j.jclinepi.2019.12.021]

\section{Stata [Computer program]}

Stata. Version 15. College Station, TX, USA: StataCorp, 2017. Available at www.stata.com.

\section{Steingart 2014}

Steingart KR, Schiller I, Horne DJ, Pai M, Boehme CC, Dendukuri N. Xpert ${ }^{\circledR}$ MTB/RIF assay for pulmonary tuberculosis and rifampicin resistance in adults. Cochrane Database of Systematic Reviews 2014, Issue 1. Art. No: CD009593. [DOI: 10.1002/14651858.CD009593.pub3]

\section{Unitaid 2017}

Boyle D. Tuberculosis Diagnostics Technology and Market Landscape. 5th edition. Vernier: World Health Organization Unitaid Secretariat, 2017.

\section{UN Sustainable Development Goals 2030}

United Nations General Assembly. Transforming our world: the 2030 agenda for sustainable development. Resolution adopted by the General Assembly on 25 September 2015. https://sustainabledevelopment.un.org/post2015/ transformingourworld/publication (accessed 22 July 2020).

\section{van't Hoog 2013}

van't Hoog AH, Langendam MW, Mitchell E, Cobelens FG, Sinclair D, Leeflang MM, et al. A systematic review of the sensitivity and specificity of symptom- and chestradiography screening for active pulmonary tuberculosis in HIV-negative persons and persons with unknown HIV status. Report to the World Health Organization. 2013. who.int/tb/ Review2Accuracyofscreeningtests.pdf?ua=1 (accessed 23 June 2020).

\section{Whiting 2011}

Whiting PF, Rutjes AW, Westwood ME, Mallett S, Deeks JJ, Reitsma JB, et al. QUADAS-2: a revised tool for the quality assessment of diagnostic accuracy studies. Annals of Internal Medicine 2011;155(8):529-36.

\section{WHO 2011}

World Health Organization. Automated real-time nucleic acid amplification technology for rapid and simultaneous detection of tuberculosis and rifampicin resistance: Xpert MTB/RIF system for the diagnosis of pulmonary and extrapulmonary TB in adults and children. apps.who.int/iris/handle/10665/112472 (accessed 18 November 2020).

\section{WHO 2016}

World Health Organization. Xpert MTB/RIF assay for the diagnosis of TB: meeting report 2016. apps.who.int/iris/ handle/10665/250383 (accessed 10 October 2020).

\section{WHO Compendium of WHO guidelines 2018}

World Health Organanization. Compendium of WHO guidelines and associated standards: ensuring optimum delivery of the cascade of care for patients with tuberculosis. Second edition - June 2018. https://www.who.int/tb/publications/ Compendium_WHO_guidelines_TB_2017/en/ (accessed 10 September 2020).

\section{WHO Consolidated Guidelines (Module 3) 2020}

World Health Organization. World Health Organization. WHO consolidated guidelines on tuberculosis. Module 3: diagnosis - rapid diagnostics for tuberculosis detection; June 2020. who.int/publications/i/item/who-consolidated-guidelineson-tuberculosis-module-3-diagnosis---rapid-diagnostics-fortuberculosis-detection (accessed 1 July 2020).

\section{WHO Consolidated Guidelines (Module 4) 2020}

World Health Organization. WHO consolidated guidelines on tuberculosis. Module 4: treatment - drug-resistant tuberculosis

Xpert MTB/RIF and Xpert Ultra assays for screening for pulmonary tuberculosis and rifampicin resistance in adults, irrespective of signs 
treatment. who.int/publications/i/item/9789240007048 (accessed 1 July 2020).

\section{WHO END TB 2015}

World Health Organization. Implementing the End TB Strategy: the essentials. WHO/HTM/TB/2015.31. who.int/tb/ publications/2015/The_Essentials_to_End_TB/en/ (accessed 23 June 2020).

\section{WHO Global TB Report 2019}

World Health Organization. Global Tuberculosis Report 2019. Geneva: World Health Organization, 2019.

\section{WHO Global TB Report 2020}

World Health Organization. Global Tuberculosis Report 2020. Licence: CC BY-NC-SA 3.0 IGO. Geneva: World Health Organization, 2020.

\section{WHO Guidelines 2016}

World Health Organization. WHO treatment guidelines for drug-resistant tuberculosis, 2016 update. October 2016 revision. apps.who.int/iris/bitstream/ handle/10665/250125/9789241549639-eng.pdf (accessed 25 November 2020).

\section{WHO LPA 2016}

World Health Organization. The use of molecular line probe assays for the detection of resistance to isoniazid and rifampicin: policy update. WHO/HTM/TB/2016.12. Geneva: World Health Organization 2016.

\section{WHO Rapid Communication 2020}

World Health Organization. Rapid communication on systematic screening for tuberculosis.. who.int/publications/i/ item/rapid-communication-on-the-systematic-screening-fortuberculosis (accessed 18 January 2021).

\section{WHO Systematic screening 2013}

World Health Organization. Systematic screening for active tuberculosis: principles and recommendations. WHO/HTM/ TB/2013.04. Geneva: World Health Organization, 2013.

\section{WHO Systematic screening 2015}

World Health Organization. Systematic screening for active tuberculosis: an operational guide WHO/HTM/TB/2015.16.

\section{CHARACTERISTICS OF STUDIES}

Characteristics of included studies [ordered by study ID] who.int/tb/publications/systematic_screening/en/ (accessed 25 November 2020).

\section{WHO Xpert MTB/RIF 2013}

World Health Organization. Automated real-time nucleic acid amplification technology for rapid and simultaneous detection of tuberculosis and rifampicin resistance: Xpert MTB/RIF assay for the diagnosis of pulmonary and extrapulmonary TB in adults and children. https://apps.who.int/iris/handle/10665/112472 (accessed 23 June 2020).

\section{WHO Xpert Ultra 2017}

World Health Organization. WHO meeting report of a technical expert consultation: non-inferiority analysis of Xpert MTB/ RIF Ultra compared to Xpert MTB/RIF. WHO/HTM/TB/2017.04. Geneva: WHO 2017.

\section{World Bank 2020}

World Bank. World bank list of economies. https:// datahelpdesk.worldbank.org/knowledgebase/articles/906519world-bank-country-and-lending-groups (accessed 18 November 2020).

\section{Zellweger 2006}

Zellweger JP, Heinzer R, Touray M, Vidondo B, Altpeter E. Intraobserver and overall agreement in the radiological assessment of tuberculosis. International Journal of Tuberculosis and Lung Diseases 2006;10(10):1123-6.

\section{Zifodya 2021}

Zifodya JS, Kreniske JS, Schiller I, Kohli M, Dendukuri N, Schumacher SG, et al. Xpert Ultra versus Xpert MTB/RIF for pulmonary tuberculosis and rifampicin resistance in adults with presumptive pulmonary tuberculosis. Cochrane Database of Systematic Reviews 2021, Issue 2. Art. No: CD009593. [DOI: 10.1002/14651858.CD009593.pub5]

\section{References to other published versions of this review}

\section{Shapiro 2020}

Shapiro AE, Ross JM, Schiller I, Kohli M, Dendukuri N, Steingart KR, et al. Xpert MTB/RIF and Xpert Ultra assays for pulmonary tuberculosis and rifampicin resistance in adults irrespective of signs or symptoms of pulmonary tuberculosis. Cochrane Database of Systematic Reviews 2020, Issue 7. Art. No: CD013694. [DOI: 10.1002/14651858.CD013694]

\section{Al-Darraji 2013}

\section{Study characteristics}

Patient Sampling

Cross-sectional design, consecutive enrolment, prospective data collection

Patient characteristics and setting Presenting signs and symptoms: not reported; HIV-positive prisoners were screened 
Age: mean 37 years (standard deviation (SD) 6.6)

Sex, female: $10 \%$

HIV infection: $100 \%$

History of TB: $29 \%$

Sample size: 125

Clinical setting: outpatient, point of care

Laboratory level: other, prison

Country: Malaysia

World Bank Income Classification: middle income

High TB burden country: no

High MDR-TB burden country: no

High TB/HIV burden country: no

Prevalence of TB cases in the study: $12.0 \%$

Index tests Index test: Xpert MTB/RIF

Target condition and reference standard(s)

Target condition: pulmonary TB

Reference standard for pulmonary TB: MGIT 960

Speciation: yes

Target condition: rifampicin resistance

Reference standard for rifampicin resistance: MGIT 960, MTBDRplus for confirmation

Flow and timing

Comparative

Notes

Methodological quality

\begin{tabular}{llll}
\hline Item & $\begin{array}{l}\text { Authors' judge- } \\
\text { ment }\end{array}$ & Risk of bias & $\begin{array}{l}\text { Applicability con- } \\
\text { cerns }\end{array}$
\end{tabular}

\section{DOMAIN 1: Patient Selection}

\begin{tabular}{lll}
\hline Was a consecutive or random sample of patients enrolled? & Yes \\
\hline Was a case-control design avoided? & Yes \\
\hline Did the study avoid inappropriate exclusions? & Yes & Low risk \\
\hline Could the selection of patients have introduced bias? & \\
\hline
\end{tabular}

Are there concerns that the included patients and setting do not match the review question? 
Al-Darraji 2013 (Continued)

\title{
DOMAIN 2: Index Test (Xpert MTB/RIF)
}

Were the index test results interpreted without knowledge of Yes the results of the reference standard?

If a threshold was used, was it pre-specified? Yes

Could the conduct or interpretation of the index test have Low risk
introduced bias?

Are there concerns that the index test, its conduct, or interpretation differ from the review question?

\section{DOMAIN 2: Index Test (Xpert Ultra)}

\section{DOMAIN 3: Reference Standard}

Is the reference standards likely to correctly classify the target Yes condition?

Were the reference standard results interpreted without knowl- Yes edge of the results of the index tests?
Could the reference standard, its conduct, or its interpreta-
Low risk tion have introduced bias?
Are there concerns that the target condition as defined by the reference standard does not match the question?

\section{DOMAIN 4: Flow and Timing}

Was there an appropriate interval between index test and refer- Yes ence standard?

\begin{tabular}{lll}
\hline Did all patients receive the same reference standard? & Yes \\
\hline Were all patients included in the analysis? & Yes \\
\hline Could the patient flow have introduced bias? & Low risk \\
\hline
\end{tabular}

\section{Al-Darraji 2016}

\section{Study characteristics}

$\begin{array}{ll}\text { Patient Sampling } & \begin{array}{l}\text { Cross-sectional design, consecutive enrolment, prospective data } \\ \text { collection }\end{array}\end{array}$

Patient characteristics and setting

\author{
Presenting signs and symptoms: prisoners were screened irre- \\ spective of s/sx; $59 \%$ reported at least 1 WHO sx \\ Age: all >=18; mean age 36.4, SD 9.8 years \\ Sex, female: $19 \%$ \\ HIV infection: $29 \%$
}

Xpert MTB/RIF and Xpert Ultra assays for screening for pulmonary tuberculosis and rifampicin resistance in adults, irrespective of signs 
History of TB: $12 \%$

Sample size: 442

Clinical setting: outpatient, prison, point of care

Laboratory level: other, prison

Country: Malaysia

World Bank Income Classification: middle income

High TB burden country: no

High MDR-TB burden country: no

High TB/HIV burden country: no

Prevalence of TB cases in the study: $6.8 \%$

\begin{tabular}{ll}
\hline Index tests & Index test: Xpert MTB/RIF \\
\hline Target condition and reference standard(s) & Target condition: pulmonary TB \\
& Reference standard for pulmonary TB: MGIT 960 \\
& Speciation: yes
\end{tabular}

Flow and timing

\section{Comparative}

Notes

4 patients with Xpert MTB/RIF+ results had neg culture results, categorized as TB cases based on clinical \& CXR findings

\section{Methodological quality}

\begin{tabular}{llll}
\hline Item & $\begin{array}{l}\text { Authors' judge- } \\
\text { ment }\end{array}$ & Risk of bias & $\begin{array}{l}\text { Applicability con- } \\
\text { cerns }\end{array}$ \\
\hline
\end{tabular}

\section{DOMAIN 1: Patient Selection}

\begin{tabular}{|c|c|c|}
\hline Was a consecutive or random sample of patients enrolled? & Yes & \\
\hline Was a case-control design avoided? & Yes & \\
\hline Did the study avoid inappropriate exclusions? & Yes & \\
\hline Could the selection of patients have introduced bias? & Low risk & \\
\hline $\begin{array}{l}\text { Are there concerns that the included patients and setting do } \\
\text { not match the review question? }\end{array}$ & & Low concern \\
\hline
\end{tabular}

Were the index test results interpreted without knowledge of Yes the results of the reference standard?

Yes 
Al-Darraji 2016 (Continued)

Could the conduct or interpretation of the index test have introduced bias?

Are there concerns that the index test, its conduct, or inter-

Low concern pretation differ from the review question?

\section{DOMAIN 2: Index Test (Xpert Ultra)}

\section{DOMAIN 3: Reference Standard}

Is the reference standards likely to correctly classify the target Yes condition?

Were the reference standard results interpreted without knowl- Yes edge of the results of the index tests?
Could the reference standard, its conduct, or its interpreta-
tion have introduced bias?
Low risk

Are there concerns that the target condition as defined by
the reference standard does not match the question?

\section{DOMAIN 4: Flow and Timing}

Was there an appropriate interval between index test and refer- Yes ence standard?

\begin{tabular}{lll}
\hline Did all patients receive the same reference standard? & Yes \\
\hline Were all patients included in the analysis? & Yes & Low risk \\
\hline Could the patient flow have introduced bias? & \\
\hline
\end{tabular}

\section{Balcha 2014}

\section{Study characteristics}

Patient Sampling

Cross-sectional design, consecutive enrolment, prospective data collection

Patient characteristics and setting

Presenting signs and symptoms: HIV-positive people screened for TB irrespective of symptoms

Age: 18 years and older, median 32 years (IQR 28 to 40 )

Sex, female: $59 \%$

HIV infection: $100 \%$

History of TB: $6 \%$

Sample size: 810

Clinical setting: outpatient

Laboratory level: intermediate

Country: Ethiopia

Xpert MTB/RIF and Xpert Ultra assays for screening for pulmonary tuberculosis and rifampicin resistance in adults, irrespective of signs

Copyright $\odot 2021$ The Authors. Cochrane Database of Systematic Reviews published by John Wiley \& Sons, Ltd. on behalf of The Cochrane

Collaboration. 
Balcha 2014 (Continued)

World Bank Income Classification: low income

High TB burden country: yes

High MDR-TB burden country: yes

High TB/HIV burden country: yes

Prevalence of TB cases in the study: $15.0 \%$

\begin{tabular}{ll}
\hline Index tests & Index: Xpert MTB/RIF \\
\hline Target condition and reference standard(s) & Target condition: pulmonary TB \\
& Reference standard for pulmonary TB: MGIT 960 \\
& Speciation: yes
\end{tabular}

Flow and timing

\section{Comparative}

Notes

$2 \%$ of participants were on anti-TB treatment for up to 2 weeks -

risk of bias for participant selection

\begin{tabular}{llll}
\hline Methodological quality & & \\
\hline Item & $\begin{array}{l}\text { Authors' judge- } \\
\text { ment }\end{array}$ & Risk of bias & $\begin{array}{l}\text { Applicability con- } \\
\text { cerns }\end{array}$ \\
\hline
\end{tabular}

DOMAIN 1: Patient Selection

\begin{tabular}{lll}
\hline Was a consecutive or random sample of patients enrolled? & Yes \\
\hline Was a case-control design avoided? & Yes & \\
\hline Did the study avoid inappropriate exclusions? & Low risk \\
\hline Could the selection of patients have introduced bias? & Unclear \\
\hline $\begin{array}{l}\text { Are there concerns that the included patients and setting do } \\
\text { not match the review question? }\end{array}$
\end{tabular}

\section{DOMAIN 2: Index Test (Xpert MTB/RIF)}

Were the index test results interpreted without knowledge of Yes the results of the reference standard?

If a threshold was used, was it pre-specified?

Yes

Could the conduct or interpretation of the index test have Low risk
introduced bias?

Are there concerns that the index test, its conduct, or interpretation differ from the review question?

DOMAIN 2: Index Test (Xpert Ultra)

\section{DOMAIN 3: Reference Standard}

Xpert MTB/RIF and Xpert Ultra assays for screening for pulmonary tuberculosis and rifampicin resistance in adults, irrespective of signs

Copyright $\odot 2021$ The Authors. Cochrane Database of Systematic Reviews published by John Wiley \& Sons, Ltd. on behalf of The Cochrane

Collaboration. 
Balcha 2014 (Continued)

Is the reference standards likely to correctly classify the target Yes condition?

Were the reference standard results interpreted without knowl- Yes edge of the results of the index tests?

Could the reference standard, its conduct, or its interpreta- Low risk
tion have introduced bias?
tion have introduced bias?

Are there concerns that the target condition as defined by
the reference standard does not match the question?

\section{DOMAIN 4: Flow and Timing}

Was there an appropriate interval between index test and refer- Yes ence standard?

\begin{tabular}{ll}
\hline Did all patients receive the same reference standard? Yes \\
\hline
\end{tabular}

Were all patients included in the analysis? Yes

Could the patient flow have introduced bias? Low risk

\section{Beyanga 2018}

\section{Study characteristics}

Patient Sampling

Patient characteristics and setting
Cross-sectional, consecutive enrolment, prospective data collection

Presenting signs and symptoms: participants were contacts of 93 pulmonary TB patients, irrespective of symptoms

Age: all ages, median 22 years (IQR 15 to 37 )

Sex, female: $57 \%$

HIV infection: unknown

History of TB: not reported

Sample size: 456

Clinical setting: outpatient

Laboratory level: intermediate

Country: Tanzania

World Bank Income Classification: low income

High TB burden country: yes

High MDR-TB burden country: no

High TB/HIV burden country: yes

Prevalence of TB cases in the study: $4 \%$ 
Beyanga 2018 (Continued)

Index tests Index: Xpert MTB/RIF

Target condition and reference standard(s)

Target condition: pulmonary TB

Reference standard: LJ

Speciation: yes

Flow and timing

Comparative

Notes 10 samples had invalid Xpert MTB/RIF results, 16 results contaminated culture.

Methodological quality

\begin{tabular}{llll}
\hline Item & $\begin{array}{l}\text { Authors' judge- } \\
\text { ment }\end{array}$ & Risk of bias & $\begin{array}{l}\text { Applicability con- } \\
\text { cerns }\end{array}$ \\
\hline
\end{tabular}

\section{DOMAIN 1: Patient Selection}

Was a consecutive or random sample of patients enrolled?

Yes

Was a case-control design avoided? Yes

Did the study avoid inappropriate exclusions? Yes

Could the selection of patients have introduced bias?

Low risk

Are there concerns that the included patients and setting do

Unclear not match the review question?

\section{DOMAIN 2: Index Test (Xpert MTB/RIF)}

Were the index test results interpreted without knowledge of Yes the results of the reference standard?

If a threshold was used, was it pre-specified? Yes

Could the conduct or interpretation of the index test have Low risk
introduced bias?
introduced bias?

Are there concerns that the index test, its conduct, or interpretation differ from the review question?

\section{DOMAIN 2: Index Test (Xpert Ultra)}

\section{DOMAIN 3: Reference Standard}

Is the reference standards likely to correctly classify the target Yes condition?

Were the reference standard results interpreted without knowl- Yes edge of the results of the index tests? 
Beyanga 2018 (Continued)

Could the reference standard, its conduct, or its interpreta-

Low risk tion have introduced bias?

Are there concerns that the target condition as defined by

Low concern the reference standard does not match the question?

\section{DOMAIN 4: Flow and Timing}

Was there an appropriate interval between index test and refer- Yes ence standard?

\begin{tabular}{ll}
\hline Did all patients receive the same reference standard? & Yes \\
\hline Were all patients included in the analysis? & No \\
\hline Could the patient flow have introduced bias? & Low risk
\end{tabular}

Bjerrum 2016

\section{Study characteristics}

Patient Sampling

Cross-sectional design, consecutive enrolment, prospective data collection

Patient characteristics and setting

Presenting signs and symptoms: HIV-infected adults screened for pulmonary TB irrespective of symptoms

Age: 18 years and older, median 38 years (IQR 31 to 45 )

Sex, female: $64 \%$

HIV infection: $100 \%$

History of TB: $6 \%$

Sample size: 195

Clinical setting: both outpatient and inpatient

Laboratory level: central

Country: Ghana

World Bank Income Classification: middle income

High TB burden country: no

High MDR-TB burden country: no

High TB/HIV burden country: yes

Prevalence of TB cases in the study: 17.9\%

\begin{tabular}{ll}
\hline Index tests & Index: Xpert MTB/RIF \\
\hline Target condition and reference standard(s) & Target condition: pulmonary TB \\
& Reference standard for pulmonary TB: LJ and MGIT 960
\end{tabular}

Xpert MTB/RIF and Xpert Ultra assays for screening for pulmonary tuberculosis and rifampicin resistance in adults, irrespective of signs

Copyright $\odot 2021$ The Authors. Cochrane Database of Systematic Reviews published by John Wiley \& Sons, Ltd. on behalf of The Cochrane Collaboration. 
Bjerrum 2016 (Continued)

Speciation: yes

Flow and timing

Comparative

\begin{tabular}{llll}
\hline Notes & Screening study & \\
\hline Methodological quality & & \\
\hline Item & $\begin{array}{l}\text { Authors'judge- } \\
\text { ment }\end{array}$ & Risk of bias & $\begin{array}{l}\text { Applicability con- } \\
\text { cerns }\end{array}$ \\
\hline
\end{tabular}

DOMAIN 1: Patient Selection

\begin{tabular}{ll}
\hline Was a consecutive or random sample of patients enrolled? & Yes \\
\hline Was a case-control design avoided? & Yes \\
\hline
\end{tabular}

Did the study avoid inappropriate exclusions?

Yes

Could the selection of patients have introduced bias? Low risk

Are there concerns that the included patients and setting do not match the review question?

Low concern

\section{DOMAIN 2: Index Test (Xpert MTB/RIF)}

Were the index test results interpreted without knowledge of Yes the results of the reference standard?

If a threshold was used, was it pre-specified?

Yes

Could the conduct or interpretation of the index test have L L risk
introduced bias?
introduced bias?

Are there concerns that the index test, its conduct, or interpretation differ from the review question?

DOMAIN 2: Index Test (Xpert Ultra)

\section{DOMAIN 3: Reference Standard}

Is the reference standards likely to correctly classify the target Yes condition?

Were the reference standard results interpreted without knowl- Yes edge of the results of the index tests?

Could the reference standard, its conduct, or its interpreta-

tion have introduced bias?

Are there concerns that the target condition as defined by

Low concern the reference standard does not match the question?

\section{DOMAIN 4: Flow and Timing}


Bjerrum 2016 (Continued)

Was there an appropriate interval between index test and refer- Yes ence standard?

Did all patients receive the same reference standard? Yes

Were all patients included in the analysis? Yes

Could the patient flow have introduced bias?

Low risk

Dorman 2012

\section{Study characteristics}

Patient Sampling $\quad \begin{aligned} & \text { Cross-sectional design, consecutive enrolment, prospective data } \\ & \text { collection }\end{aligned}$
collection

\section{Patient characteristics and setting}

Presenting signs and symptoms: miners attending occupational health services for annual examination, irrespective of signs and symptoms

Age: 43 years (34-49)

Sex, female: $6.1 \%$

HIV infection: $14 \%$ positive, $50 \%$ unknown

History of TB: $12 \%$

Sample size: 6893

Clinical setting: outpatient (mine)

Laboratory level: intermediate

Country: South Africa

World Bank Income Classification: middle income

High TB burden country: yes

High MDR-TB burden country: yes

High TB/HIV burden country: yes

\begin{tabular}{ll}
\hline Index tests & Index test: Xpert MTB/RIF \\
\hline Target condition and reference standard(s) & Target condition: pulmonary TB \\
& $\begin{array}{l}\text { Reference standard: MGIT } 960 \\
\text { Speciation: yes }\end{array}$ \\
\hline Flow and timing & $\begin{array}{l}272 / 6893(3.9 \%) \text { specimens had invalid Xpert MTB/RIF or contam- } \\
\text { inated culture; although not included, these participants were ac- } \\
\text { counted for }\end{array}$ \\
\end{tabular}

\section{Comparative}

\section{Notes}

Xpert MTB/RIF and Xpert Ultra assays for screening for pulmonary tuberculosis and rifampicin resistance in adults, irrespective of signs

Copyright $\odot 2021$ The Authors. Cochrane Database of Systematic Reviews published by John Wiley \& Sons, Ltd. on behalf of The Cochrane Collaboration. 
Dorman 2012 (Continued)

Methodological quality

\begin{tabular}{llll}
\hline Item & $\begin{array}{l}\text { Authors' judge- } \\
\text { ment }\end{array}$ & Risk of bias & $\begin{array}{l}\text { Applicability con- } \\
\text { cerns }\end{array}$ \\
\hline
\end{tabular}

\section{DOMAIN 1: Patient Selection}

Was a consecutive or random sample of patients enrolled?

Was a case-control design avoided?

Did the study avoid inappropriate exclusions?
Yes

Yes

Yes

Could the selection of patients have introduced bias?

Low risk

Are there concerns that the included patients and setting do

Low concern not match the review question?

\section{DOMAIN 2: Index Test (Xpert MTB/RIF)}

Were the index test results interpreted without knowledge of Yes the results of the reference standard?

If a threshold was used, was it pre-specified?

Yes

\section{Could the conduct or interpretation of the index test have} introduced bias?

\section{Are there concerns that the index test, its conduct, or inter-} pretation differ from the review question?

\section{DOMAIN 2: Index Test (Xpert Ultra)}

\section{DOMAIN 3: Reference Standard}

Is the reference standards likely to correctly classify the target Yes condition?

Were the reference standard results interpreted without knowl- Yes edge of the results of the index tests?

Could the reference standard, its conduct, or its interpretation have introduced bias?

Are there concerns that the target condition as defined by the reference standard does not match the question?

\section{DOMAIN 4: Flow and Timing}

Was there an appropriate interval between index test and refer- Yes ence standard?

Did all patients receive the same reference standard? Yes

Were all patients included in the analysis?

Yes

\section{Could the patient flow have introduced bias?}

Low risk

Xpert MTB/RIF and Xpert Ultra assays for screening for pulmonary tuberculosis and rifampicin resistance in adults, irrespective of signs

Copyright $\odot 2021$ The Authors. Cochrane Database of Systematic Reviews published by John Wiley \& Sons, Ltd. on behalf of The Cochrane Collaboration. 
Heidebrecht 2016

\section{Study characteristics}

Patient Sampling

Cross-sectional, consecutive enrolment, prospective data collection

Patient characteristics and setting

Presenting signs and symptoms: irrespective of symptoms. 45\% had signs and symptoms of TB.

Age: adults median 41 years (IQR 31-57)

Sex, female: $65 \%$

HIV infection: $62 \%$

History of TB: $23 \%$

Sample size: 215

Clinical setting: inpatient medical ward

Laboratory level: intermediate

Country: South Africa

World Bank Income Classification: middle income

High TB burden country: yes

High MDR-TB burden country: yes

High TB/HIV burden country: yes

Prevalence of TB cases in the study: 23\%

\begin{tabular}{ll}
\hline Index tests & Index test: Xpert MTB/RIF \\
\hline Target condition and reference standard(s) & $\begin{array}{l}\text { Target condition: pulmonary TB } \\
\text { Reference standard for pulmonary TB: MGIT 960 and Middlebrook } \\
\text { Speciation: yes }\end{array}$ \\
\hline Flow and timing & $\begin{array}{l}\text { Of 215 patients with Xpert, only } 125 \text { also had culture performed } \\
\text { (on 2nd sample). 2nd sample for reference testing unobtainable } \\
\text { for nearly 40\% of participants. Total data come from 125 pairs. }\end{array}$ \\
\hline Comparative & $\begin{array}{l}\text { 6/27 Xpert MTB/RIF negative patients were diagnosed with extra- } \\
\text { pulmonary TB }\end{array}$ \\
\hline Notes & $\begin{array}{l}\text { Authors' judge- } \\
\text { ment }\end{array}$ \\
\hline Item & Risk of bias
\end{tabular}

\section{DOMAIN 1: Patient Selection}

Was a consecutive or random sample of patients enrolled?

Yes

Xpert MTB/RIF and Xpert Ultra assays for screening for pulmonary tuberculosis and rifampicin resistance in adults, irrespective of signs

Copyright $\odot 2021$ The Authors. Cochrane Database of Systematic Reviews published by John Wiley \& Sons, Ltd. on behalf of The Cochrane Collaboration. 
Heidebrecht 2016 (Continued)

Was a case-control design avoided?

Did the study avoid inappropriate exclusions?

Yes

Could the selection of patients have introduced bias?

Low risk

Are there concerns that the included patients and setting do

High not match the review question?

\section{DOMAIN 2: Index Test (Xpert MTB/RIF)}

Were the index test results interpreted without knowledge of Yes the results of the reference standard?

If a threshold was used, was it pre-specified?

Yes

Could the conduct or interpretation of the index test have Low risk
introduced bias?

Could the conduct or interpretation of the index test have

Are there concerns that the index test, its conduct, or interpretation differ from the review question?

DOMAIN 2: Index Test (Xpert Ultra)

\section{DOMAIN 3: Reference Standard}

Is the reference standards likely to correctly classify the target Yes condition?

Were the reference standard results interpreted without knowl- Yes edge of the results of the index tests?

\section{Could the reference standard, its conduct, or its interpreta- Low risk tion have introduced bias?}

Are there concerns that the target condition as defined by
the reference standard does not match the question?

\section{DOMAIN 4: Flow and Timing}

Was there an appropriate interval between index test and refer- Yes ence standard?

Did all patients receive the same reference standard?

Yes

Were all patients included in the analysis?

No

\section{Could the patient flow have introduced bias?}

High risk

Henostroza 2016

\section{Study characteristics}


Henostroza 2016 (Continued)

Patient Sampling

Patient characteristics and setting

Cross-sectional design, consecutive enrolment, prospective data collection

Presenting signs and symptoms: ART-naïve people presenting for initiation of HIV care

Age: 16 years and older, median 34 years (IQR 29 to 40 )

Sex, female: $49 \%$

HIV infection: $100 \%$

History of TB: not reported

Sample size: 332

Clinical setting: outpatient

Laboratory level: central

Country: Zambia

World Bank Income Classification: middle income

High TB burden country: yes

High MDR-TB burden country: no

High TB/HIV burden country: yes

Prevalence of TB cases in the study: $18.6 \%$

\begin{tabular}{ll}
\hline Index tests & Index: Xpert MTB/RIF \\
\hline Target condition and reference standard(s) & Target condition: pulmonary TB \\
& Reference standard for pulmonary TB: LJ and MGIT 960 \\
& Speciation: yes
\end{tabular}

Flow and timing

Comparative

Notes

The paper states that outpatients in this cohort were likely to have been less ill than hospitalized patients

\section{Methodological quality}

\begin{tabular}{|c|c|c|c|}
\hline Item & $\begin{array}{l}\text { Authors' judge- } \\
\text { ment }\end{array}$ & Risk of bias & $\begin{array}{l}\text { Applicability con- } \\
\text { cerns }\end{array}$ \\
\hline
\end{tabular}

DOMAIN 1: Patient Selection

\begin{tabular}{lll}
\hline Was a consecutive or random sample of patients enrolled? & Yes \\
\hline Was a case-control design avoided? & Yes \\
\hline Did the study avoid inappropriate exclusions? & Yes \\
\hline Could the selection of patients have introduced bias? & Low risk \\
\hline
\end{tabular}

Xpert MTB/RIF and Xpert Ultra assays for screening for pulmonary tuberculosis and rifampicin resistance in adults, irrespective of signs 50 or symptoms (Review)

Copyright (C) 2021 The Authors. Cochrane Database of Systematic Reviews published by John Wiley \& Sons, Ltd. on behalf of The Cochrane Collaboration. 
Henostroza 2016 (Continued)

Are there concerns that the included patients and setting do not match the review question?

\section{DOMAIN 2: Index Test (Xpert MTB/RIF)}

Were the index test results interpreted without knowledge of Yes the results of the reference standard?

If a threshold was used, was it pre-specified? Yes

Could the conduct or interpretation of the index test have Low risk
introduced bias?
introduced bias?

Are there concerns that the index test, its conduct, or interpretation differ from the review question?

\section{DOMAIN 2: Index Test (Xpert Ultra)}

\section{DOMAIN 3: Reference Standard}

Is the reference standards likely to correctly classify the target Yes condition?

Were the reference standard results interpreted without knowl- Yes edge of the results of the index tests?
Could the reference standard, its conduct, or its interpreta-
Low risk tion have introduced bias?

Are there concerns that the target condition as defined by the reference standard does not match the question?

\section{DOMAIN 4: Flow and Timing}

Was there an appropriate interval between index test and refer- Yes ence standard?

Did all patients receive the same reference standard? Yes

Were all patients included in the analysis? Yes

\section{Study characteristics}

Patient Sampling

Cross-sectional design, consecutive enrolment, prospective data collection

Patient characteristics and setting

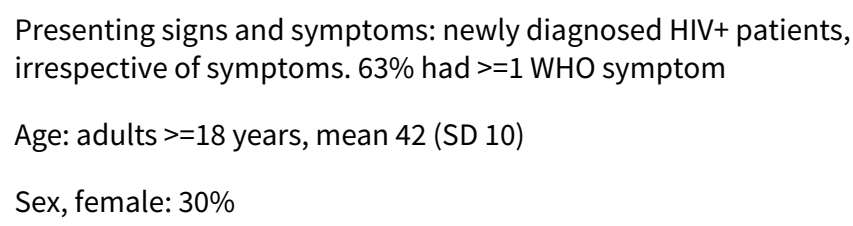

Xpert MTB/RIF and Xpert Ultra assays for screening for pulmonary tuberculosis and rifampicin resistance in adults, irrespective of signs or symptoms (Review)

Copyright $\odot 2021$ The Authors. Cochrane Database of Systematic Reviews published by John Wiley \& Sons, Ltd. on behalf of The Cochrane Collaboration. 
HIV infection: $100 \%$, median CD4 count: 122 cells/mm3

History of TB: $3 \%$

Sample size: 103 (131 enrolled, 103 provided sputum)

Clinical setting: outpatient

Laboratory level: intermediate

Country: Georgia

World Bank Income Classification: upper-middle income

High TB burden country: no

High MDR-TB burden country: no

High TB/HIV burden country: no

Prevalence of TB cases in the study: $12 \%$

\begin{tabular}{ll}
\hline Index tests & Index test: Xpert MTB/RIF \\
\hline Target condition and reference standard(s) & Target condition: pulmonary TB \\
& Reference standard for pulmonary TB: LJ solid culture \\
& Speciation: yes
\end{tabular}

Flow and timing

Comparative

Notes

\section{Methodological quality}

\begin{tabular}{llll}
\hline Item & $\begin{array}{l}\text { Authors' judge- } \\
\text { ment }\end{array}$ & Risk of bias & $\begin{array}{l}\text { Applicability con- } \\
\text { cerns }\end{array}$
\end{tabular}

DOMAIN 1: Patient Selection

\begin{tabular}{lll}
\hline Was a consecutive or random sample of patients enrolled? & Yes \\
\hline Was a case-control design avoided? & Yes & Low risk \\
\hline Did the study avoid inappropriate exclusions? & \\
\hline Could the selection of patients have introduced bias? & Low concern \\
\hline $\begin{array}{l}\text { Are there concerns that the included patients and setting do } \\
\text { not match the review question? }\end{array}$
\end{tabular}

\section{DOMAIN 2: Index Test (Xpert MTB/RIF)}

Were the index test results interpreted without knowledge of 
Kempker 2019 (Continued)

Could the conduct or interpretation of the index test have

Low risk introduced bias?

Are there concerns that the index test, its conduct, or inter-

Low concern pretation differ from the review question?

\section{DOMAIN 2: Index Test (Xpert Ultra)}

\section{DOMAIN 3: Reference Standard}

Is the reference standards likely to correctly classify the target Yes condition?

Were the reference standard results interpreted without knowl- Yes edge of the results of the index tests?
Could the reference standard, its conduct, or its interpreta- tion have introduced bias?

Are there concerns that the target condition as defined by
the reference standard does not match the question?

\section{DOMAIN 4: Flow and Timing}

Was there an appropriate interval between index test and refer- Yes ence standard?

\begin{tabular}{ll}
\hline Did all patients receive the same reference standard? & Yes \\
\hline Were all patients included in the analysis? & Yes \\
\hline Could the patient flow have introduced bias? & Low risk \\
\hline
\end{tabular}

\section{LaCourse 2016}

\section{Study characteristics}

\section{Patient Sampling}

Patient characteristics and setting
Cross-sectional design, consecutive enrolment, prospective data collection

Presenting signs and symptoms: none reported. HIV-infected women accessing prevention of mother-to-child transmission services as part of antenatal care were eligible

Age: 16 years and older, median 25 years (IQR 22 to 30 )

Sex, female: $100 \%$

HIV infection: $100 \%$

History of TB: $9 \%$

Sample size: 288

Clinical setting: outpatient

Laboratory level: central 
Country: Kenya

World Bank Income Classification: middle income

High TB burden country: yes

High MDR-TB burden country: yes

High TB/HIV burden country: yes

Prevalence of TB cases in the study: $2.4 \%$

\begin{tabular}{ll}
\hline Index tests & Index test: Xpert MTB/RIF \\
\hline Target condition and reference standard(s) & Target condition: pulmonary TB \\
& Reference standard for pulmonary TB: MGIT 960 \\
& Speciation: yes \\
\hline
\end{tabular}

Flow and timing

Comparative

Notes

Methodological quality

\begin{tabular}{llll}
\hline Item & $\begin{array}{l}\text { Authors' judge- } \\
\text { ment }\end{array}$ & Risk of bias & $\begin{array}{l}\text { Applicability con- } \\
\text { cerns }\end{array}$ \\
\hline
\end{tabular}

\section{DOMAIN 1: Patient Selection}

\begin{tabular}{lll}
\hline Was a consecutive or random sample of patients enrolled? & Yes \\
\hline Was a case-control design avoided? & Yes & Unclear \\
\hline Did the study avoid inappropriate exclusions? & \\
\hline Could the selection of patients have introduced bias? & Low risk \\
\hline
\end{tabular}

Are there concerns that the included patients and setting do
not match the review question?

DOMAIN 2: Index Test (Xpert MTB/RIF)

Were the index test results interpreted without knowledge of Yes the results of the reference standard?

If a threshold was used, was it pre-specified? Yes

Could the conduct or interpretation of the index test have Low risk
introduced bias?

Are there concerns that the index test, its conduct, or interpretation differ from the review question?

\section{DOMAIN 2: Index Test (Xpert Ultra)}


LaCourse 2016 (Continued)

\section{DOMAIN 3: Reference Standard}

Is the reference standards likely to correctly classify the target Yes condition?

Were the reference standard results interpreted without knowl- Yes

edge of the results of the index tests?

\section{Could the reference standard, its conduct, or its interpreta- \\ Low risk \\ tion have introduced bias?}

Are there concerns that the target condition as defined by

Low concern the reference standard does not match the question?

\section{DOMAIN 4: Flow and Timing}

Was there an appropriate interval between index test and refer- Yes ence standard?

Did all patients receive the same reference standard? Yes

Were all patients included in the analysis? Yes

\section{Study characteristics}

Patient Sampling

Patient characteristics and setting
Cross-sectional design, consecutive enrolment, prospective data collection

Presenting signs and symptoms: HIV-infected people with advanced immunodeficiency, irrespective of symptoms (most had 1 or more of the following TB symptoms: current cough, fever, night sweats, or weight loss)

Age: median 34 years (IQR 28 to 41)

Sex, female: $65.4 \%$

HIV infection: $100 \%$

History of TB: $26.5 \%$

Sample size: 394

Clinical setting: HIV anti-retroviral clinic; all participants were screened for TB

Laboratory level: central

Country: South Africa, Cape Town

World Bank Income Classification: middle income

High TB burden country: yes

High MDR-TB burden country: yes 
Lawn 2011 (Continued)

High TB/HIV burden country: yes

TB incidence rate: 993 per 100,000

MDR-TB prevalence: \% MDR-TB among new TB cases $=0.9 \%$

(Source: survey in Western Cape Province, 2002) and among retreatment cases $=4.0 \%$ (Source: survey in Western Cape Province, 2002)

Prevalence of TB cases in the study: $18.3 \%$

\begin{tabular}{ll}
\hline Index tests & Xpert MTB/RIF \\
\hline Target condition and reference standard(s) & $\begin{array}{l}\text { Target condition: rifampicin resistance } \\
\text { Reference standard for rifampicin resistance: culture-based DST } \\
\text { (MGIT 960) }\end{array}$ \\
\hline
\end{tabular}

Flow and timing

Comparative

Notes

Lawn 2011 determined Xpert MTB/RIF accuracy for detection of ri-

fampicin resistance, with respect to culture-based drug susceptibil-

ity testing. Xpert MTB/RIF accuracy for detection of TB, with respect

to culture, is reported in Lawn 2012.

\section{Methodological quality}

\begin{tabular}{llll}
\hline Item & $\begin{array}{l}\text { Authors' judge- } \\
\text { ment }\end{array}$ & Risk of bias & $\begin{array}{l}\text { Applicability con- } \\
\text { cerns }\end{array}$ \\
\hline
\end{tabular}

\section{DOMAIN 1: Patient Selection}

\begin{tabular}{lll}
\hline Was a consecutive or random sample of patients enrolled? & Yes \\
\hline Was a case-control design avoided? & Yes & \\
\hline Did the study avoid inappropriate exclusions? & Yes & Low risk \\
\hline Could the selection of patients have introduced bias? & \\
\hline
\end{tabular}

Are there concerns that the included patients and setting
do not match the review question?

DOMAIN 2: Index Test (Xpert MTB/RIF)

Were the index test results interpreted without knowledge of Yes the results of the reference standard?

\begin{tabular}{l}
\hline If a threshold was used, was it pre-specified? \\
$\begin{array}{l}\text { Could the conduct or interpretation of the index test have } \\
\text { introduced bias? }\end{array}$ \\
\hline $\begin{array}{l}\text { Are there concerns that the index test, its conduct, or in- } \\
\text { terpretation differ from the review question? }\end{array}$ \\
\hline DOMAIN 2: Index Test (Xpert Ultra)
\end{tabular}

Xpert MTB/RIF and Xpert Ultra assays for screening for pulmonary tuberculosis and rifampicin resistance in adults, irrespective of signs 
Lawn 2011 (Continued)

\section{DOMAIN 3: Reference Standard}

Is the reference standards likely to correctly classify the target Yes condition?

Were the reference standard results interpreted without Yes knowledge of the results of the index tests?
Could the reference standard, its conduct, or its interpre-
Low risk
tation have introduced bias?

\section{Are there concerns that the target condition as defined by the reference standard does not match the question?}

Low concern

\section{DOMAIN 4: Flow and Timing}

Was there an appropriate interval between index test and ref- Yes erence standard?

Did all patients receive the same reference standard? Yes

Were all patients included in the analysis? Yes

Could the patient flow have introduced bias?

Low risk

Lawn 2012

\section{Study characteristics}

Patient Sampling

Cross-sectional design, consecutive enrolment, prospective data collection

Patient characteristics and setting

Presenting signs and symptoms: HIV-infected people with advanced immunodeficiency, irrespective of symptoms (most had 1 or more of the following TB symptoms: current cough, fever, night sweats, or weight loss)

Age: median 34 years (IQR 28 to 41 )

Sex, female: $65.4 \%$

HIV infection: $100 \%$

History of TB: $26.5 \%$

Sample size: 394

Clinical setting: HIV anti-retroviral clinic; all participants were screened for TB

Laboratory level: central

Country: South Africa, Cape Town

World Bank Income Classification: middle income

High TB burden country: yes

High MDR-TB burden country: yes

High TB/HIV burden country: yes 
MDR-TB prevalence: $\%$ MDR-TB among new TB cases $=0.9 \%$ (Source: survey in Western Cape Province, 2002) and among retreatment cases $=4.0 \%$ (Source: survey in Western Cape Province, 2002)

Prevalence of TB cases in the study: $18.3 \%$

\begin{tabular}{ll}
\hline Index tests & Index test: Xpert MTB/RIF \\
\hline Target condition and reference standard(s) & Target condition: pulmonary TB \\
& Reference standard for pulmonary TB: MGIT 960 \\
& Speciation: yes \\
& Target condition: rifampicin resistance \\
& Reference standard for rifampicin resistance: MGIT 960
\end{tabular}

Flow and timing

Comparative

\section{Notes}

This study evaluated the use of Xpert MTB/RIF to screen HIV-infected people with advanced immunodeficiency enrolling in antiretroviral therapy services regardless of symptoms, although most participants in the study had TB symptoms. Of 3 participants with apparent false-positive Xpert MTB/RIF results, on follow-up 2 had overt pulmonary and systemic symptoms suggestive of TB and improved on anti-TB treatment. The 3rd participant was lost to follow-up. Median CD4 cell count, 171 cells/ml; IQR 102 to 236

\section{Methodological quality}

\begin{tabular}{llll}
\hline Item & Authors' judgement & Risk of bias & $\begin{array}{l}\text { Applicability con- } \\
\text { cerns }\end{array}$ \\
\hline
\end{tabular}

\section{DOMAIN 1: Patient Selection}

Was a consecutive or random sample of patients en- Yes rolled?

\begin{tabular}{ll}
\hline Was a case-control design avoided? & Yes \\
\hline Did the study avoid inappropriate exclusions? & Yes \\
\hline
\end{tabular}

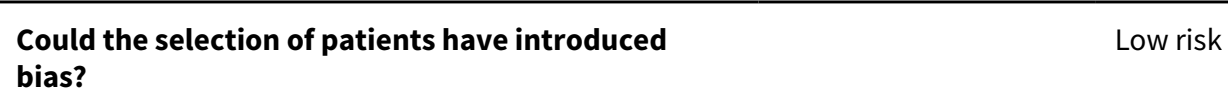

Are there concerns that the included patients and
setting do not match the review question?

\section{DOMAIN 2: Index Test (Xpert MTB/RIF)}

Were the index test results interpreted without knowl- Yes

edge of the results of the reference standard?

If a threshold was used, was it pre-specified? Yes


Lawn 2012 (Continued)
Could the conduct or interpretation of the index test
Low risk have introduced bias?

Are there concerns that the index test, its conduct,

Low concern or interpretation differ from the review question?

\section{DOMAIN 2: Index Test (Xpert Ultra)}

\section{DOMAIN 3: Reference Standard}

Is the reference standards likely to correctly classify the Yes target condition?

Were the reference standard results interpreted with- Yes out knowledge of the results of the index tests?
Could the reference standard, its conduct, or its in- terpretation have introduced bias?

\section{Are there concerns that the target condition as de- fined by the reference standard does not match the} question?

\section{DOMAIN 4: Flow and Timing}

Was there an appropriate interval between index test Yes and reference standard?

\begin{tabular}{lll}
\hline Did all patients receive the same reference standard? & Yes \\
\hline Were all patients included in the analysis? & Yes & Low risk \\
\hline Could the patient flow have introduced bias? & & \\
\hline
\end{tabular}

Lopez-Varela 2019

\section{Study characteristics}

Patient Sampling

Patient characteristics and setting
Cross-sectional design, consecutive enrolment, prospective data collection

Presenting signs and symptoms: HIV-infected people at the time of HIV diagnosis, irrespective of symptoms $(41.3 \%$ had 1 or more TB symptoms: current cough, fever, night sweats, or weight loss)

Age: mean 35 years (SD 14)

Sex, female: $56.4 \%$

HIV infection: 100\% (median CD4 328, IQR 195 to 505)

History of TB: $1.6 \%$

Sample size: 91

Clinical setting: home-based HIV counselling and testing 
Country: Mozambique

World Bank Income Classification: low income

High TB burden country: yes

High MDR-TB burden country: yes

High TB/HIV burden country: yes

TB incidence rate: 847 per 100,000

Prevalence of TB cases in the study: $4.4 \%$

\begin{tabular}{ll}
\hline Index tests & Index test: Xpert MTB/RIF \\
\hline Target condition and reference standard(s) & Target condition: pulmonary TB \\
& Reference standard: liquid culture \\
& Speciation: yes \\
\hline
\end{tabular}

Flow and timing

Comparative

Notes

Methodological quality

\begin{tabular}{|c|c|c|c|}
\hline Item & $\begin{array}{l}\text { Authors' judge- } \\
\text { ment }\end{array}$ & Risk of bias & $\begin{array}{l}\text { Applicability con- } \\
\text { cerns }\end{array}$ \\
\hline
\end{tabular}

\section{DOMAIN 1: Patient Selection}

\begin{tabular}{lll}
\hline Was a consecutive or random sample of patients enrolled? & Yes \\
\hline Was a case-control design avoided? & Yes \\
\hline Did the study avoid inappropriate exclusions? & Yes \\
\hline Could the selection of patients have introduced bias? & Low risk
\end{tabular}

Are there concerns that the included patients and setting do

Low concern not match the review question?

\section{DOMAIN 2: Index Test (Xpert MTB/RIF)}

Were the index test results interpreted without knowledge of Yes the results of the reference standard?

If a threshold was used, was it pre-specified? Yes

\section{Could the conduct or interpretation of the index test have introduced bias? \\ Low risk}

\section{Are there concerns that the index test, its conduct, or inter- pretation differ from the review question?}


Lopez-Varela 2019 (Continued)

DOMAIN 2: Index Test (Xpert Ultra)

\title{
DOMAIN 3: Reference Standard
}

Is the reference standards likely to correctly classify the target Yes condition?

Were the reference standard results interpreted without knowl- Yes edge of the results of the index tests?

\section{Could the reference standard, its conduct, or its interpreta- Low risk} tion have introduced bias?

\section{Are there concerns that the target condition as defined by the reference standard does not match the question?

\section{DOMAIN 4: Flow and Timing}

Was there an appropriate interval between index test and refer- Yes ence standard?

\begin{tabular}{ll}
\hline Did all patients receive the same reference standard? Yes \\
\hline
\end{tabular}

Were all patients included in the analysis? Yes

\section{Could the patient flow have introduced bias?}

Low risk

Mollel 2017

\section{Study characteristics}

\section{Patient Sampling}

Cross-sectional design, consecutive enrolment, prospective data collection

\section{Patient characteristics and setting}

\author{
Presenting signs and symptoms: not reported \\ Age: 16 years and older, mean 42 years \\ Sex, female: $55 \%$ \\ HIV infection: $100 \%$ \\ History of TB: not reported \\ Sample size: 69 \\ Clinical setting: outpatient \\ Laboratory level: intermediate \\ Country: Tanzania \\ World Bank Income Classification: low income \\ High TB burden country: yes \\ High MDR-TB burden country: no \\ High TB/HIV burden country: yes
}


Mollel 2017 (Continued)

Prevalence of TB cases in the study: $13.0 \%$

\begin{tabular}{ll}
\hline Index tests & Index test: Xpert MTB/RIF \\
\hline Target condition and reference standard(s) & Target condition: pulmonary TB \\
& Reference standard for pulmonary TB: LJ \\
& Speciation: not reported
\end{tabular}

Flow and timing

Comparative

Notes

Methodological quality

\begin{tabular}{|c|c|c|c|}
\hline Item & $\begin{array}{l}\text { Authors' judge- } \\
\text { ment }\end{array}$ & Risk of bias & $\begin{array}{l}\text { Applicability con- } \\
\text { cerns }\end{array}$ \\
\hline
\end{tabular}

DOMAIN 1: Patient Selection

\begin{tabular}{ll}
\hline Was a consecutive or random sample of patients enrolled? & Yes \\
\hline Was a case-control design avoided? & Yes \\
\hline Did the study avoid inappropriate exclusions? & Yes
\end{tabular}

Could the selection of patients have introduced bias?

Low risk

Are there concerns that the included patients and setting do

Low concern not match the review question?

\section{DOMAIN 2: Index Test (Xpert MTB/RIF)}

Were the index test results interpreted without knowledge of Yes the results of the reference standard?

If a threshold was used, was it pre-specified? Yes

Could the conduct or interpretation of the index test have Low risk
introduced bias?
introduced bias?

Are there concerns that the index test, its conduct, or interpretation differ from the review question?

\section{DOMAIN 2: Index Test (Xpert Ultra)}

\section{DOMAIN 3: Reference Standard}

Is the reference standards likely to correctly classify the target Yes condition?

Were the reference standard results interpreted without knowl- Unclear edge of the results of the index tests? 
Mollel 2017 (Continued)

Could the reference standard, its conduct, or its interpreta-

Unclear risk tion have introduced bias?

Are there concerns that the target condition as defined by

Unclear the reference standard does not match the question?

\section{DOMAIN 4: Flow and Timing}

Was there an appropriate interval between index test and refer- Yes ence standard?

Did all patients receive the same reference standard? Yes

Were all patients included in the analysis? Yes

Could the patient flow have introduced bias? Low risk

Ntinginya 2012

\section{Study characteristics}

Patient Sampling

Cross-sectional study, consecutive enrolment, prospective data collection

Patient characteristics and setting

Presenting signs and symptoms: household contacts enrolled irrespective of symptoms (15.5\% reported >=1 TB symptom)

Age: age $>=5$, mean 26 years (SD 17.6)

Sex, female: $59.4 \%$

HIV infection: not assessed

History of TB: $3 \%$

Sample size: 33

Clinical setting: community-based household contacts of TB patients

Laboratory level: intermediate

Country: Tanzania

World Bank Income Classification: low income

High TB burden country: yes

High MDR-TB burden country: no

High TB/HIV burden country: yes

Prevalence of TB cases in the study: 15\% of people who produced sputum; $2.3 \%$ of contacts overall 
Ntinginya 2012 (Continued)

Speciation: yes

Flow and timing

219 contacts approached; only $33(15.1 \%)$ able to produce sputum and included in results

Comparative

Notes

\section{Methodological quality}

\begin{tabular}{llll}
\hline Item & $\begin{array}{l}\text { Authors' judge- } \\
\text { ment }\end{array}$ & Risk of bias & $\begin{array}{l}\text { Applicability con- } \\
\text { cerns }\end{array}$
\end{tabular}

\section{DOMAIN 1: Patient Selection}

\begin{tabular}{ll}
\hline Was a consecutive or random sample of patients enrolled? & Yes \\
\hline Was a case-control design avoided? & Yes \\
\hline Did the study avoid inappropriate exclusions? & Yes
\end{tabular}

\begin{tabular}{ll}
\hline Could the selection of patients have introduced bias? & Low risk \\
\hline $\begin{array}{l}\text { Are there concerns that the included patients and setting do } \\
\text { not match the review question? }\end{array}$ & Unclear \\
\hline
\end{tabular}

DOMAIN 2: Index Test (Xpert MTB/RIF)

Were the index test results interpreted without knowledge of Yes the results of the reference standard?

If a threshold was used, was it pre-specified? Yes

Could the conduct or interpretation of the index test have Low risk
introduced bias?

introduced bias?

Are there concerns that the index test, its conduct, or interpretation differ from the review question?

\section{DOMAIN 2: Index Test (Xpert Ultra)}

\section{DOMAIN 3: Reference Standard}

Is the reference standards likely to correctly classify the target Yes condition?

Were the reference standard results interpreted without knowl- Yes edge of the results of the index tests?
Could the reference standard, its conduct, or its interpreta-
Low risk tion have introduced bias?

Are there concerns that the target condition as defined by
the reference standard does not match the question?

DOMAIN 4: Flow and Timing

Xpert MTB/RIF and Xpert Ultra assays for screening for pulmonary tuberculosis and rifampicin resistance in adults, irrespective of signs 
Ntinginya 2012 (Continued)

Was there an appropriate interval between index test and refer- Yes ence standard?

\begin{tabular}{ll}
\hline Did all patients receive the same reference standard? & Uncle \\
\hline Were all patients included in the analysis? & No \\
\hline
\end{tabular}

\section{Could the patient flow have introduced bias?}

High risk

\section{O'Grady 2012}

\section{Study characteristics}

\section{Patient Sampling}

\section{Patient characteristics and setting}

Cross-sectional sampling, consecutive enrolment, prospective data collection

Presenting signs and symptoms: adult medical inpatients at tertiary hospital, regardless of symptoms or admission diagnosis (on TB treatment excluded from analysis)

Age: adults $>15$ years, median age 35 years (IQR 28 to 43 )

HIV infection: $71 \%$

History of TB: unknown

Sample size: 643

Clinical setting: tertiary hospital admitted patients

Laboratory level: intermediate

Country: Zambia

World Bank Income Classification: low income

High TB burden country: yes

High MDR-TB burden country: no

High TB/HIV burden country: yes

Prevalence of TB cases in the study: $31 \%$ of 643 patients not on treatment

\begin{tabular}{ll}
\hline Index tests & Index test: Xpert MTB/RIF \\
\hline Target condition and reference standard(s) & Target condition: pulmonary TB \\
& Reference standard: MGIT 960 \\
& Speciatiation: yes
\end{tabular}

Flow and timing

881 admitted patients contributed to demographics above, of whom 643 not already on TB treatment and included in the analysis of sensitivity and specificity of Xpert MTB/RIF

\section{Comparative}

\section{Notes}

Xpert MTB/RIF and Xpert Ultra assays for screening for pulmonary tuberculosis and rifampicin resistance in adults, irrespective of signs

Copyright $\odot 2021$ The Authors. Cochrane Database of Systematic Reviews published by John Wiley \& Sons, Ltd. on behalf of The Cochrane Collaboration. 
O'Grady 2012 (Continued)

Methodological quality

\begin{tabular}{llll}
\hline Item & $\begin{array}{l}\text { Authors' judge- } \\
\text { ment }\end{array}$ & Risk of bias & $\begin{array}{l}\text { Applicability con- } \\
\text { cerns }\end{array}$ \\
\hline
\end{tabular}

DOMAIN 1: Patient Selection

Was a consecutive or random sample of patients enrolled?

Yes

Was a case-control design avoided? Yes

Did the study avoid inappropriate exclusions?

Yes

Could the selection of patients have introduced bias?

Low risk

Are there concerns that the included patients and setting do

High

not match the review question?

\section{DOMAIN 2: Index Test (Xpert MTB/RIF)}

Were the index test results interpreted without knowledge of Yes

the results of the reference standard?

If a threshold was used, was it pre-specified? Yes

Could the conduct or interpretation of the index test have Low risk
introduced bias?

introduced bias?

Are there concerns that the index test, its conduct, or interpretation differ from the review question?

\section{DOMAIN 2: Index Test (Xpert Ultra)}

\section{DOMAIN 3: Reference Standard}

Is the reference standards likely to correctly classify the target Yes condition?

Were the reference standard results interpreted without knowl- Yes

edge of the results of the index tests?

Could the reference standard, its conduct, or its interpreta-

Low risk

tion have introduced bias?

Are there concerns that the target condition as defined by

Low concern the reference standard does not match the question?

\section{DOMAIN 4: Flow and Timing}

Was there an appropriate interval between index test and refer- Yes ence standard?

Did all patients receive the same reference standard? Yes

Were all patients included in the analysis? Yes

Could the patient flow have introduced bias?

Low risk

Xpert MTB/RIF and Xpert Ultra assays for screening for pulmonary tuberculosis and rifampicin resistance in adults, irrespective of signs

Copyright $\odot 2021$ The Authors. Cochrane Database of Systematic Reviews published by John Wiley \& Sons, Ltd. on behalf of The Cochrane

Collaboration. 


\section{Study characteristics}

$\begin{array}{ll}\text { Patient Sampling } & \begin{array}{l}\text { Cross-sectional study, consecutive enrolment, prospective data } \\ \text { collection }\end{array}\end{array}$

Patient characteristics and setting

Presenting signs and symptoms: HIV+ adults initiating ART, irrespective of signs and symptoms. (52\% reported at least 1 symptom)

Sex, female: $62 \%$

HIV infection: $100 \%$

History of TB: not reported

Sample size: 571

Clinical setting: outpatient ART clinic

Laboratory level: intermediate

Country: South Africa

World Bank Income Classification: middle income

High TB burden country: yes

High MDR-TB burden country: yes

High TB/HIV burden country: yes

Prevalence of TB cases in the study: 12\%

\begin{tabular}{ll}
\hline Index tests & Index test: Xpert MTB/RIF \\
\hline Target condition and reference standard(s) & Target condition: pulmonary TB \\
& Reference standard: MGIT 960 \\
\hline
\end{tabular}

Flow and timing

Comparative

Notes

Methodological quality

\begin{tabular}{|c|c|c|c|}
\hline Item & $\begin{array}{l}\text { Authors' judge- } \\
\text { ment }\end{array}$ & Risk of bias & $\begin{array}{l}\text { Applicability con- } \\
\text { cerns }\end{array}$ \\
\hline
\end{tabular}

\section{DOMAIN 1: Patient Selection}

\begin{tabular}{lll}
\hline Was a consecutive or random sample of patients enrolled? & Yes \\
\hline Was a case-control design avoided? & Yes \\
\hline Did the study avoid inappropriate exclusions? & Yes & Low risk \\
\hline Could the selection of patients have introduced bias? & \\
\hline
\end{tabular}


Reeve 2019a (Continued)

Are there concerns that the included patients and setting do not match the review question?

\section{DOMAIN 2: Index Test (Xpert MTB/RIF)}

Were the index test results interpreted without knowledge of Yes the results of the reference standard?

If a threshold was used, was it pre-specified? Yes

Could the conduct or interpretation of the index test have Low risk
introduced bias?
introduced bias?

Are there concerns that the index test, its conduct, or interpretation differ from the review question?

\section{DOMAIN 2: Index Test (Xpert Ultra)}

\section{DOMAIN 3: Reference Standard}

Is the reference standards likely to correctly classify the target Yes condition?

Were the reference standard results interpreted without knowl- Yes edge of the results of the index tests?
Could the reference standard, its conduct, or its interpreta-
Low risk tion have introduced bias?

Are there concerns that the target condition as defined by the reference standard does not match the question?

\section{DOMAIN 4: Flow and Timing}

Was there an appropriate interval between index test and refer- Yes ence standard?

Did all patients receive the same reference standard? Yes

Were all patients included in the analysis? Yes

\section{Study characteristics}

$\begin{array}{ll}\text { Patient Sampling } & \begin{array}{l}\text { Cross-sectional study, consecutive enrolment, prospective data } \\ \text { collection }\end{array}\end{array}$

Patient characteristics and setting 
HIV infection: $100 \%$

History of TB: not reported

Sample size: 571

Clinical setting: outpatient ART clinic

Laboratory level: intermediate

Country: South Africa

World Bank Income Classification: middle income

High TB burden country: yes

High MDR-TB burden country: yes

High TB/HIV burden country: yes

Prevalence of TB cases in the study: $12 \%$

\begin{tabular}{ll}
\hline Index tests & Index test: Xpert Ultra \\
\hline Target condition and reference standard(s) & Target condition: pulmonary TB \\
& Reference standard: MGIT 960 \\
\hline
\end{tabular}

Flow and timing

Comparative

Notes

\section{Methodological quality}

\begin{tabular}{|c|c|c|c|}
\hline Item & $\begin{array}{l}\text { Authors' judge- } \\
\text { ment }\end{array}$ & Risk of bias & $\begin{array}{l}\text { Applicability con- } \\
\text { cerns }\end{array}$ \\
\hline
\end{tabular}

\section{DOMAIN 1: Patient Selection}

\begin{tabular}{lll}
\hline Was a consecutive or random sample of patients enrolled? & Yes \\
\hline Was a case-control design avoided? & Yes & Yes \\
\hline Did the study avoid inappropriate exclusions? & \\
\hline Could the selection of patients have introduced bias?
\end{tabular}

\section{Are there concerns that the included patients and setting do not match the review question?}

DOMAIN 2: Index Test (Xpert MTB/RIF)

\section{DOMAIN 2: Index Test (Xpert Ultra)}

Were the index test results interpreted without knowledge of the results of the reference standard?

If a threshold was used, was it pre-specified?
Yes

Yes 
Reeve 2019b (Continued)

Could the conduct or interpretation of the index test have

Low risk introduced bias?

Are there concerns that the index test, its conduct, or inter-

Low concern pretation differ from the review question?

\section{DOMAIN 3: Reference Standard}

Is the reference standards likely to correctly classify the target Yes condition?

Were the reference standard results interpreted without knowl- Yes edge of the results of the index tests?

Could the reference standard, its conduct, or its interpreta- L
tion have introduced bias?
tion have introduced bias?

Are there concerns that the target condition as defined by

Low concern the reference standard does not match the question?

\section{DOMAIN 4: Flow and Timing}

Was there an appropriate interval between index test and refer- Yes ence standard?

\begin{tabular}{ll}
\hline Did all patients receive the same reference standard? & Yes \\
\hline Were all patients included in the analysis? & Yes \\
\hline Could the patient flow have introduced bias? & Low risk
\end{tabular}

Santos 2020

\section{Study characteristics}

Patient Sampling

Cross-sectional study, consecutive enrolment, prospective data collection

Patient characteristics and setting

Presenting signs and symptoms: Adults residing in prisons, irrespective of signs and symptoms. ( $40 \%$ of total pop reported at least $1 \mathrm{sx}, 80.4 \%$ of sputum providers reported at least 1 symptom)

Sex, female: $0 \%$

HIV infection: not reported ( $2 \%$ in TB+)

History of TB: $8 \%$ of screened population

Sample size: $\mathrm{N}=5387 ; \mathrm{N}=1385$ with both Xpert \& culture performed (remainder couldn't make sputum)

Clinical setting: prisons

Laboratory level: intermediate

Country: Brazil 
High TB burden country: yes

High MDR-TB burden country: no

High TB/HIV burden country: yes

Prevalence of TB cases in the study: 7\% (of persons tested/producing sputum)

\begin{tabular}{ll}
\hline Index tests & Xpert MTB/RIF \\
\hline Target condition and reference standard(s) & $\begin{array}{l}\text { Target condition: pulmonary TB } \\
\text { Reference standard: TB culture (both MGIT \& LJ available at refer- } \\
\text { ence lab) }\end{array}$ \\
\end{tabular}

Flow and timing

\section{Comparative}

Notes

3919 unable to produce sputum. 1467 (27\%) provided sputum, 1385 participants had both Xpert \& culture performed. Invalid/not done tests not included in analysis. Does not specify whether Mtb species identification performed on TB culture.

\section{Methodological quality}

\begin{tabular}{llll}
\hline Item & $\begin{array}{l}\text { Authors' judge- } \\
\text { ment }\end{array}$ & Risk of bias & $\begin{array}{l}\text { Applicability con- } \\
\text { cerns }\end{array}$
\end{tabular}

DOMAIN 1: Patient Selection

\begin{tabular}{lll}
\hline Was a consecutive or random sample of patients enrolled? & Yes \\
\hline Was a case-control design avoided? & Yes & Yes \\
\hline Did the study avoid inappropriate exclusions? & & Low risk \\
\hline Could the selection of patients have introduced bias? & Low concern \\
\hline $\begin{array}{l}\text { Are there concerns that the included patients and setting do } \\
\text { not match the review question? }\end{array}$
\end{tabular}

\section{DOMAIN 2: Index Test (Xpert MTB/RIF)}

Were the index test results interpreted without knowledge of the results of the reference standard?

If a threshold was used, was it pre-specified?

Could the conduct or interpretation of the index test have Low risk
introduced bias?

Are there concerns that the index test, its conduct, or interpretation differ from the review question? 
Santos 2020 (Continued)

\section{DOMAIN 3: Reference Standard}

Is the reference standards likely to correctly classify the target Yes condition?

Were the reference standard results interpreted without knowl- Yes

edge of the results of the index tests?

Could the reference standard, its conduct, or its interpreta- Low risk
tion have introduced bias?

Are there concerns that the target condition as defined by

Low concern the reference standard does not match the question?

\section{DOMAIN 4: Flow and Timing}

Was there an appropriate interval between index test and refer- Yes ence standard?

Did all patients receive the same reference standard? Yes

Were all patients included in the analysis? No

Could the patient flow have introduced bias?

High risk

Tahseen 2018

\section{Study characteristics}

Patient Sampling

Cross-sectional study, consecutive enrolment, prospective data collection

Patient characteristics and setting

Presenting signs and symptoms: irrespective of symptoms

Age: 18 years and older, median 30 (26-46)

Sex, female: $0 \%$

HIV infection: $100 \%$

History of TB: $9 \%$

Sample size: 635

Clinical setting: outpatient IVDU treatment centre

Laboratory level: intermediate

Country: Pakistan

World Bank Income Classification: middle income

High TB burden country: yes

High MDR-TB burden country: yes

High TB/HIV burden country: no 
Tahseen 2018 (Continued)

Prevalence of TB cases in the study: $13.0 \%$

\begin{tabular}{|c|c|}
\hline Index tests & Index test: Xpert MTB/RIF \\
\hline \multirow[t]{3}{*}{ Target condition and reference standard(s) } & Target condition: pulmonary TB \\
\hline & Reference standard: LJ and MGIT 960 \\
\hline & Speciaiton: yes \\
\hline Flow and timing & $\begin{array}{l}12 \text { invalid Xpert; } 30 \text { contaminated cultures in } 635 \text { participants, not } \\
\text { included in analysis }\end{array}$ \\
\hline \multicolumn{2}{|l|}{ Comparative } \\
\hline \multicolumn{2}{|l|}{ Notes } \\
\hline \multicolumn{2}{|l|}{ Methodological quality } \\
\hline Item & $\begin{array}{l}\text { Applicability con- } \\
\text { cerns }\end{array}$ \\
\hline
\end{tabular}

\section{DOMAIN 1: Patient Selection}

\begin{tabular}{lcl}
\hline Was a consecutive or random sample of patients enrolled? & Yes \\
\hline Was a case-control design avoided? & Yes & Yes \\
\hline Did the study avoid inappropriate exclusions? & & Low risk \\
\hline Could the selection of patients have introduced bias? & \\
\hline
\end{tabular}

\section{Are there concerns that the included patients and setting do not match the review question?}

\section{DOMAIN 2: Index Test (Xpert MTB/RIF)}

\section{Were the index test results interpreted without knowledge of Yes} the results of the reference standard?

\begin{tabular}{lll}
\hline If a threshold was used, was it pre-specified? & Yes \\
$\begin{array}{l}\text { Could the conduct or interpretation of the index test have } \\
\text { introduced bias? }\end{array}$ & Low risk
\end{tabular}

\section{Are there concerns that the index test, its conduct, or inter- pretation differ from the review question?}

\section{DOMAIN 2: Index Test (Xpert Ultra)}

\section{DOMAIN 3: Reference Standard}

Is the reference standards likely to correctly classify the target Yes condition?

Were the reference standard results interpreted without knowl- Yes edge of the results of the index tests? 
Tahseen 2018 (Continued)

Could the reference standard, its conduct, or its interpretation have introduced bias?

Are there concerns that the target condition as defined by

Low concern the reference standard does not match the question?

\section{DOMAIN 4: Flow and Timing}

Was there an appropriate interval between index test and refer- Yes ence standard?

\begin{tabular}{ll}
\hline Did all patients receive the same reference standard? & Yes \\
\hline Were all patients included in the analysis? & Yes \\
\hline Could the patient flow have introduced bias? & Low risk
\end{tabular}

Yoon 2017

\section{Study characteristics}

Patient Sampling

Cross-sectional design, consecutive enrolment, prospective data collection

Patient characteristics and setting

Presenting signs and symptoms: HIV-positive people initiating antiretroviral therapy

Age: 18 years and older, median 33 years (IQR 27 to 40)

Sex, female: $53 \%$

HIV infection: $100 \%$

History of TB: $4 \%$

Sample size: 1177

Clinical setting: outpatient HIV/AIDS clinics

Laboratory level: central

Country: Uganda

World Bank Income Classification: middle income

High TB burden country: no

High MDR-TB burden country: no

High TB/HIV burden country: yes

Prevalence of TB cases in the study: $13.8 \%$

\begin{tabular}{ll}
\hline Index tests & Index test: Xpert MTB/RIF \\
\hline Target condition and reference standard(s) & Target condition: pulmonary TB \\
& Reference standard for pulmonary TB: LJ and MGIT 960
\end{tabular}

Xpert MTB/RIF and Xpert Ultra assays for screening for pulmonary tuberculosis and rifampicin resistance in adults, irrespective of signs 74 or symptoms (Review)

Copyright $\odot 2021$ The Authors. Cochrane Database of Systematic Reviews published by John Wiley \& Sons, Ltd. on behalf of The Cochrane Collaboration. 
Yoon 2017 (Continued)

Flow and timing

Comparative

Notes

Methodological quality

\begin{tabular}{llll}
\hline Item & $\begin{array}{l}\text { Authors' judge- } \\
\text { ment }\end{array}$ & Risk of bias & $\begin{array}{l}\text { Applicability con- } \\
\text { cerns }\end{array}$ \\
\hline
\end{tabular}

DOMAIN 1: Patient Selection

Was a consecutive or random sample of patients enrolled? Yes

Was a case-control design avoided? Yes

Did the study avoid inappropriate exclusions? Yes

Could the selection of patients have introduced bias? Low risk

Are there concerns that the included patients and setting do

Low concern not match the review question?

\section{DOMAIN 2: Index Test (Xpert MTB/RIF)}

Were the index test results interpreted without knowledge of Yes

the results of the reference standard?

If a threshold was used, was it pre-specified?

Yes

Could the conduct or interpretation of the index test have Low risk
introduced bias?

introduced bias?

Are there concerns that the index test, its conduct, or interpretation differ from the review question?

DOMAIN 2: Index Test (Xpert Ultra)

\section{DOMAIN 3: Reference Standard}

Is the reference standards likely to correctly classify the target Yes condition?

Were the reference standard results interpreted without knowl- Yes edge of the results of the index tests?

Could the reference standard, its conduct, or its interpreta-

Low risk

tion have introduced bias?

Are there concerns that the target condition as defined by

Low concern the reference standard does not match the question?

\section{DOMAIN 4: Flow and Timing}

Xpert MTB/RIF and Xpert Ultra assays for screening for pulmonary tuberculosis and rifampicin resistance in adults, irrespective of signs 
Yoon 2017 (Continued)

Was there an appropriate interval between index test and refer- Yes ence standard?

Did all patients receive the same reference standard?

Yes

Were all patients included in the analysis?

Yes

\section{Could the patient flow have introduced bias?}

Low risk

ART: antiretroviral therapy; IQR: interquartile range; MDR: multidrug-resistant; MGIT: Mycobacteria Growth Indicator Tube; SD: standard deviation; TB: tuberculosis.

Characteristics of excluded studies [ordered by study ID]

\begin{tabular}{|c|c|}
\hline Study & Reason for exclusion \\
\hline Adams 2015 & persons not screened irrespective of symptoms \\
\hline Adejumo 2018 & persons not screened irrespective of symptoms \\
\hline Adetunji 2019 & no microbiological reference standard (TB culture) \\
\hline Agizew 2017 & persons not screened irrespective of symptoms \\
\hline Aia 2016 & persons not screened irrespective of symptoms \\
\hline Antonenka 2013 & persons not screened irrespective of symptoms \\
\hline Ardizzoni 2015 & persons not screened irrespective of symptoms \\
\hline Ardizzoni 2020 & persons not screened irrespective of symptoms \\
\hline Assefa 2019 & no microbiological reference standard (TB culture) \\
\hline Auld 2016a & not original research \\
\hline Auld 2016b & persons not screened irrespective of symptoms \\
\hline Auld 2020 & no microbiological reference standard (TB culture) \\
\hline Awan 2018 & persons not screened irrespective of symptoms \\
\hline Ayala 2016 & persons not screened irrespective of symptoms \\
\hline Bablishvili 2015 & persons not screened irrespective of symptoms \\
\hline Bacells 2016 & Xpert used on a non-respiratory sample \\
\hline Balcha 2014a & duplicate data from another study \\
\hline Balcha 2015 & duplicate data from another study \\
\hline Basir 2019 & no microbiological reference standard (TB culture) \\
\hline
\end{tabular}




\begin{tabular}{|c|c|}
\hline Study & Reason for exclusion \\
\hline Bassett 2019 & no microbiological reference standard (TB culture) \\
\hline Benjamin 2019 & persons not screened irrespective of symptoms \\
\hline Bhardwaj 2019 & persons not screened irrespective of symptoms \\
\hline Bjerrum 2015 & number of positive tests not reported \\
\hline Blakemore 2011 & persons not screened irrespective of symptoms \\
\hline Boum 2016 & persons not screened irrespective of symptoms \\
\hline Byashalira 2019 & persons not screened irrespective of symptoms \\
\hline Calligaro 2017 & data not disaggregated on persons screened with or regardless of symptoms \\
\hline Carmone 2017 & no microbiological reference standard (TB culture) \\
\hline Cavanaugh 2016 & data insufficient for $2 \times 2$ table \\
\hline Celik 2015 & persons not screened irrespective of symptoms \\
\hline Charoensook 2018 & persons not screened irrespective of symptoms \\
\hline Chry 2020 & persons not screened irrespective of symptoms \\
\hline Chumpa 2020 & persons not screened irrespective of symptoms \\
\hline Deshmukh 2020 & persons not screened irrespective of symptoms \\
\hline Ekeke 2020 & persons not screened irrespective of symptoms \\
\hline Farra 2017 & persons not screened irrespective of symptoms \\
\hline Floridia 2017 & no microbiological reference standard (TB culture) \\
\hline Gautam 2019 & persons not screened irrespective of symptoms \\
\hline Gelalcha 2017 & persons not screened irrespective of symptoms \\
\hline Gizachew 2017 & persons not screened irrespective of symptoms \\
\hline Gupta-Wright 2018 & no microbiological reference standard (TB culture) \\
\hline Gursoy 2016 & persons not screened irrespective of symptoms \\
\hline Habeenzu 2017 & persons not screened irrespective of symptoms \\
\hline Habte 2016 & persons not screened irrespective of symptoms \\
\hline Hanifa 2016 & no microbiological reference standard (TB culture) \\
\hline Head 2019 & persons not screened irrespective of symptoms \\
\hline Hiruy 2018 & persons not screened irrespective of symptoms \\
\hline
\end{tabular}

Xpert MTB/RIF and Xpert Ultra assays for screening for pulmonary tuberculosis and rifampicin resistance in adults, irrespective of signs 


\begin{tabular}{|c|c|}
\hline Study & Reason for exclusion \\
\hline Ho 2016 & no microbiological reference standard (TB culture) \\
\hline Hosseinipour 2016 & no microbiological reference standard (TB culture) \\
\hline Huang 2018 & persons not screened irrespective of symptoms \\
\hline Huerga 2020 & persons not screened irrespective of symptoms \\
\hline Huh 2019 & Xpert used on a non-respiratory sample \\
\hline Kamenska 2019 & persons not screened irrespective of symptoms \\
\hline Kerkhoff 2014 & Xpert used on a non-respiratory sample \\
\hline Kurbaniyazova 2017 & data not disaggregated on persons screened with or regardless of symptoms \\
\hline Kuyinu 2018 & persons not screened irrespective of symptoms \\
\hline LaCourse 2014 & paediatric population \\
\hline LaCourse 2018 & paediatric population \\
\hline Lawn 2012a & duplicate data from another study \\
\hline Lawn 2012b & duplicate data from another study \\
\hline Lawn 2013 & duplicate data from another study \\
\hline Lawn 2015 & data insufficient for $2 \times 2$ table \\
\hline Lawn 2017 & duplicate data from another study \\
\hline Lebina 2016 & no microbiological reference standard (TB culture) \\
\hline Lima 2020 & persons not screened irrespective of symptoms \\
\hline Luo 2019 & persons not screened irrespective of symptoms \\
\hline Maria 2018 & persons not screened irrespective of symptoms \\
\hline Marks 2019 & no microbiological reference standard (TB culture) \\
\hline Marlowe 2011 & persons not screened irrespective of symptoms \\
\hline Mbatchou 2019 & data insufficient for $2 \times 2$ table \\
\hline Mbu 2018 & data insufficient for $2 \times 2$ table \\
\hline Meng 2017 & persons not screened irrespective of symptoms \\
\hline Metcalfe 2015 & persons not screened irrespective of symptoms \\
\hline Metcalfe 2016 & persons not screened irrespective of symptoms \\
\hline Miller 2011 & persons not screened irrespective of symptoms \\
\hline
\end{tabular}

Xpert MTB/RIF and Xpert Ultra assays for screening for pulmonary tuberculosis and rifampicin resistance in adults, irrespective of signs 


\begin{tabular}{|c|c|}
\hline Study & Reason for exclusion \\
\hline Mishra 2020 & persons not screened irrespective of symptoms \\
\hline Modi 2016 & data insufficient for $2 \times 2$ table \\
\hline Morishita 2017 & persons not screened irrespective of symptoms \\
\hline Nathavitharana 2017 & persons not screened irrespective of symptoms \\
\hline Nicol 2018 & persons not screened irrespective of symptoms \\
\hline Nikolayevskyy 2019 & persons not screened irrespective of symptoms \\
\hline Ou 2019 & persons not screened irrespective of symptoms \\
\hline Ozkutuk 2014 & persons not screened irrespective of symptoms \\
\hline Parcell 2017 & persons not screened irrespective of symptoms \\
\hline Park 2013 & persons not screened irrespective of symptoms \\
\hline Pimkina 2015 & persons not screened irrespective of symptoms \\
\hline Ramamurthy 2016 & persons not screened irrespective of symptoms \\
\hline Reepalu 2016 & data insufficient for $2 \times 2$ table \\
\hline Reis 2019 & persons not screened irrespective of symptoms \\
\hline Sarinoglu 2020 & persons not screened irrespective of symptoms \\
\hline Semitala 2019 & no microbiological reference standard (TB culture) \\
\hline Shah 2019 & paediatric population \\
\hline Sun 2019 & persons not screened irrespective of symptom \\
\hline Teo 2011 & persons not screened irrespective of symptom \\
\hline Trajman 2014 & persons not screened irrespective of symptom \\
\hline van Kampen 2015 & persons not screened irrespective of symptom \\
\hline Van Rie 2011 & not original research \\
\hline Yasemin 2019 & persons not screened irrespective of symptom \\
\hline Yoon 2019 & duplicate data from another study \\
\hline
\end{tabular}

\section{A T A}

Presented below are all the data for all of the tests entered into the review. 
Table Tests. Data tables by test

\begin{tabular}{lll}
\hline Test & No. of studies & No. of participants \\
\hline 1 Xpert MTB/RIF, HIV positive, irrespective of TB symptoms & 12 & 4775 \\
\hline 2 Xpert Ultra, HIV, irrespective of TB symptoms & 1 & 571 \\
\hline 3 Xpert MTB/RIF, household contacts, irrespective of TB symptoms & 2 & 508 \\
\hline 4 Xpert MTB/RIF, prisoners, irrespective of TB symptoms & 1827 \\
\hline 5 Xpert MTB/RIF, miners, irrespective of TB symptoms & 2 & 6621 \\
\hline 6 Xpert MTB/RIF, admitted patients, irrespective of TB symptoms & 1 & 768 \\
\hline 7 Xpert MTB/RIF, all high-risk groups & 2 & 13114 \\
\hline 8 Xpert MTB/RIF for rifampicin resistance & 18 & 159 \\
\hline
\end{tabular}

\section{Test 1. Xpert MTB/RIF, HIV positive, irrespective of TB symptoms}

Xpert MTB/RIF, HIV positive, irrespective of TB symptoms

$\begin{array}{lrrrrrr}\text { Study } & \text { TP } & \text { FP } & \text { FN } & \text { TN } & \text { Sensitivity }(95 \% \mathrm{Cl}) & \text { Specificity }(95 \% \mathrm{Cl}) \text { Sensitivity (95\% Cl]Specificity (95\% Cl) } \\ \text { Al-Darraji 2013 } & 8 & 0 & 7 & 110 & 0.53[0.27,0.79] & 1.00[0.97,1.00] \\ \text { Balcha 2014 } & 81 & 13 & 41 & 677 & 0.66[0.57,0.75] & 0.98[0.97,0.99] \\ \text { Bjerrum 2016 } & 27 & 5 & 8 & 55 & 0.77[0.60,0.90] & 0.92[0.82,0.97] \\ \text { Henostroza 2016 } & 39 & 5 & 23 & 256 & 0.63[0.50,0.75] & 0.98[0.96,0.99] \\ \text { Kempker 2019 } & 9 & 3 & 3 & 88 & 0.75[0.43,0.95] & 0.97[0.91,0.99] \\ \text { LaCourse 2016 } & 3 & 1 & 4 & 280 & 0.43[0.10,0.82] & 1.00[0.98,1.00] \\ \text { Lawn 2012 } & 49 & 4 & 36 & 427 & 0.58[0.46,0.68] & 0.99[0.98,1.00] \\ \text { Lopez-Varela 2019 } & 3 & 0 & 1 & 87 & 0.75[0.19,0.99] & 1.00[0.96,1.00] \\ \text { Mollel 2017 } & 9 & 0 & 0 & 60 & 1.00[0.66,1.00] & 1.00[0.94,1.00] \\ \text { Reeve 2019a } & 33 & 1 & 35 & 502 & 0.49[0.36,0.61] & 1.00[0.99,1.00]\end{array}$

Test 2. Xpert Ultra, HIV, irrespective of TB symptoms

Xpert Ultra, HIV, irrespective of TB symptoms

Study TP FP FN TN Sensitivity (95\% Cl) Specificity $(95 \% \mathrm{Cl}$ ) Sensitivity (95\% Cl)Specificity (95\% Cl)

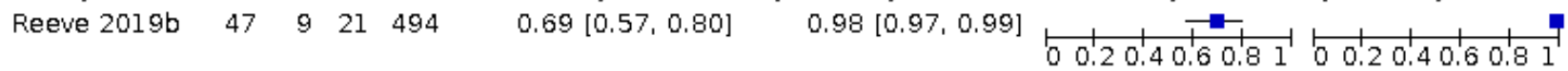

\section{Test 3. Xpert MTB/RIF, household contacts, irrespective of TB symptoms}

Xpert MTB/RIF, household contacts, irrespective of TB symptoms

Study TP FP FN TN Sensitivity $(95 \% \mathrm{Cl})$ Specificity $(95 \% \mathrm{Cl})$ Sensitivity $(95 \% \mathrm{Cl})$ Specificity $(95 \% \mathrm{Cl})$ Beyanga $2018 \quad 6 \quad 7 \quad 12 \quad 427 \quad 0.33[0.13,0.59] \quad 0.98[0.97,0.99$ Ntinginya $2012 \quad 5 \quad 0 \quad 0 \quad 51 \quad 1.00[0.48,1.00] \quad 1.00[0.93,1.00]$

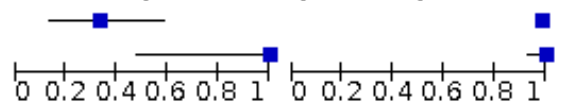


Test 4. Xpert MTB/RIF, prisoners, irrespective of TB symptoms

Xpert MTB/RIF, prisoners, irrespective of TB symptoms

Study TP FP FN TN Sensitivity $[95 \% \mathrm{Cl})$ Specificity $[95 \% \mathrm{Cl})$ Sensitivity $[95 \%$ Cl $]$ Specificity $[95 \% \mathrm{Cl})$ $\begin{array}{lrrrrrr}\text { Al-Darraji } 2016 & 16 & 4 & 14 & 408 & 0.53[0.34,0.72] & 0.99[0.98,1.00] \\ \text { Santos 2020 } & 88 & 65 & 9 & 1223 & 0.91[0.83,0.96] & 0.95[0.94,0.96]\end{array}$

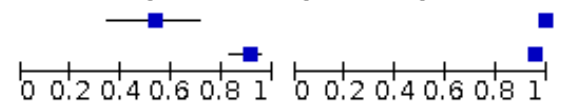

Test 5. Xpert MTB/RIF, miners, irrespective of TB symptoms

Xpert MTB/RIF, miners, irrespective of TB symptoms

Study TP FP FN TN Sensitivity $(95 \% \mathrm{Cl})$ Specificity $(95 \% \mathrm{Cl})$ Sensitivity $(95 \% \mathrm{Cl}) \mathrm{Specificity}(95 \% \mathrm{Cl})$ Dorman $2012 \quad 117 \quad 27 \quad 70 \quad 6407 \quad 0.63[0.55,0.70] \quad 1.00[0.99,1.00]$

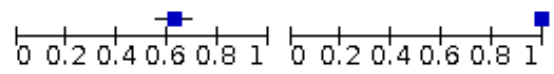

Test 6. Xpert MTB/RIF, admitted patients, irrespective of TB symptoms

Xpert MTB/RIF, admitted patients, irrespective of TB symptoms

Study TP FP FN TN Sensitivity $(95 \% \mathrm{Cl})$ Specificity $(95 \% \mathrm{Cl})$ Sensitivity $(95 \% \mathrm{Cl})$ Specificity $(95 \% \mathrm{Cl})$ Heidebrecht $2016 \quad 23 \quad 5 \quad 6 \quad 91 \quad 0.79[0.60,0.92] \quad 0.95[0.88,0.98]$ $\begin{array}{llllllll}\text { O'Grady } 2012 & 173 & 22 & 28 & 420 & 0.86[0.80,0.91] & 0.95[0.93,0.97]\end{array}$

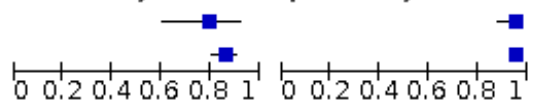

Test 7. Xpert MTB/RIF, all high-risk groups

Xpert MTB/RIF, all high-risk groups

Study

Al-Darraji 2013

Al-Darraji 2016

Balcha 2014

Beyanga 2018

Bjerrum 2016

Dorman 2012

Heidebrecht 2016

Henostroza 2016

Kempker 2019

LaCourse 2016

Lawn 2012

Lopez-Varela 2019

Mollel 2017

Ntinginya 2012

O'Grady 2012

Reeve $2019 a$

Tahseen 2018

Yoon 2017

$\begin{array}{rrrrr}\text { TP } & \text { FP } & \text { FN } & \text { TN } & \text { Sensitivity }(95 \% \mathrm{Cl}] \\ 8 & 0 & 7 & 110 & 0.53[0.27,0.79] \\ 16 & 4 & 14 & 408 & 0.53[0.34,0.72] \\ 81 & 13 & 41 & 677 & 0.66[0.57,0.75] \\ 6 & 7 & 12 & 427 & 0.33[0.13,0.59] \\ 27 & 5 & 8 & 55 & 0.77[0.60,0.90] \\ 117 & 27 & 70 & 6407 & 0.63[0.55,0.70] \\ 23 & 5 & 6 & 91 & 0.79[0.60,0.92] \\ 39 & 5 & 23 & 256 & 0.63[0.50,0.75] \\ 9 & 3 & 3 & 88 & 0.75[0.43,0.95] \\ 3 & 1 & 4 & 280 & 0.43[0.10,0.82] \\ 49 & 4 & 36 & 427 & 0.58[0.46,0.68] \\ 3 & 0 & 1 & 87 & 0.75[0.19,0.99] \\ 9 & 0 & 0 & 60 & 1.00[0.66,1.00] \\ 5 & 0 & 0 & 51 & 1.00[0.48,1.00] \\ 173 & 22 & 28 & 420 & 0.86[0.80,0.91] \\ 33 & 1 & 35 & 502 & 0.49[0.36,0.61] \\ 13 & 11 & 7 & 574 & 0.65[0.41,0.85] \\ 84 & 8 & 79 & 1006 & 0.52[0.44,0.59]\end{array}$

\begin{abstract}
$1.00[0.97,1.00]$
$0.99[0.98,1.00]$

$0.98[0.97,0.99]$

$0.98[0.97,0.99]$

$0.92[0.82,0.97]$

$1.00[0.99,1.00]$

$0.95[0.88,0.98]$

$0.98[0.96,0.99]$

$0.97[0.91,0.99]$

$1.00[0.98,1.00]$

$0.99[0.98,1.00]$

$1.00[0.96,1.00]$

$1.00[0.94,1.00]$

$1.00[0.93,1.00]$

$0.95[0.93,0.97]$

$1.00[0.99,1.00]$

$0.98[0.97,0.99]$

$0.99[0.98,1.00]$
\end{abstract}

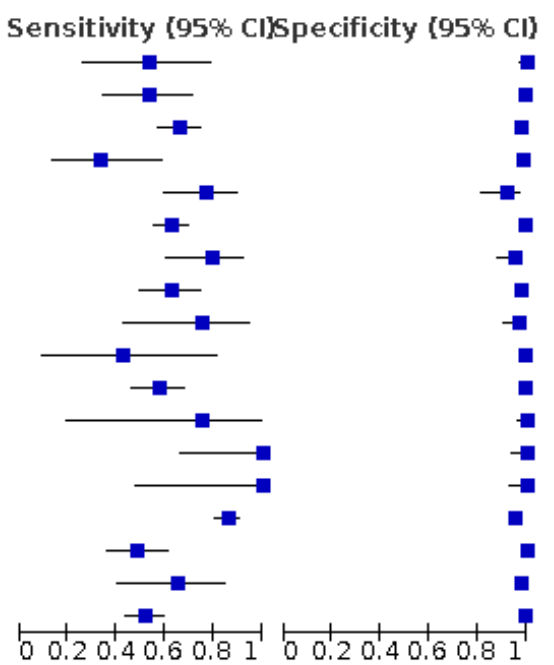




\section{Test 8. Xpert MTB/RIF for rifampicin resistance}

Xpert MTB/RIF for rifampicin resistance

Study

Al-Darraji $2013 \quad 0 \quad 0000$

$\begin{array}{lllll}\text { Lawn } 2011 & 4 & 3 & 0 & 48\end{array}$

$\begin{array}{lllll}\text { O'Grady } 2012 & 13 & 2 & 3 & 78\end{array}$
Not estimable
$.00[0.40,1.00]$

$0.81[0.54,0.96]$
Specificity $(95 \% \mathrm{Cl})$ Sensitivity $[95 \%$ Cl$\}$ Specificity $[95 \% \mathrm{CI})$
$1.00[0.63,1.00]$
$0.94[0.84,0.99]$
$0.97[0.91,1.00]$ 


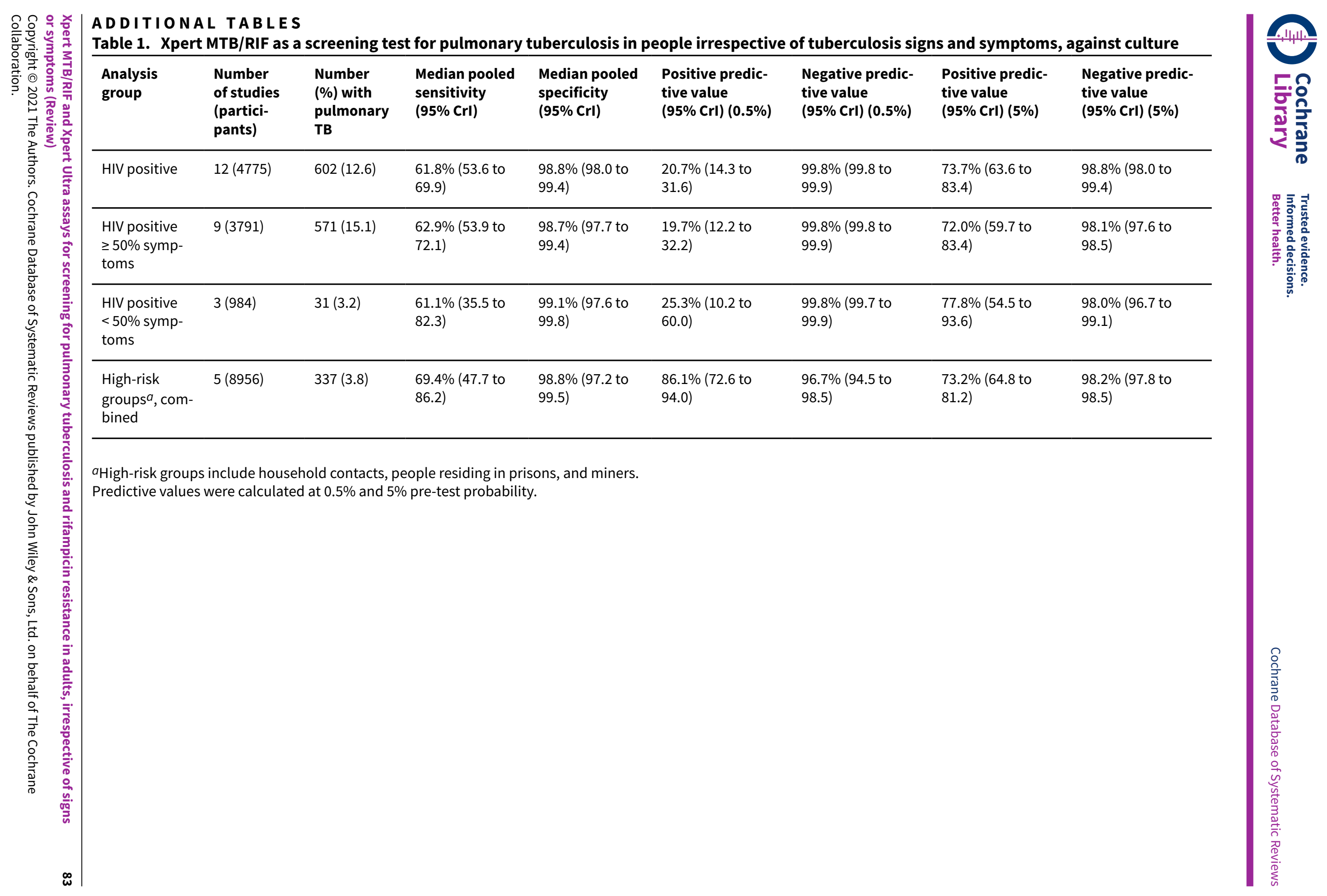




\section{AP PEN DICES}

\section{Appendix 1. Search strategy \\ MEDLINE (PubMed)}

\begin{tabular}{|c|c|}
\hline Search & Query \\
\hline$\# 1$ & Search Tuberculosis or MDR-TB or XDR-TB or tuberculous Field: Title/Abstract \\
\hline \#2 & Search "Mycobacterium tuberculosis" [Mesh] \\
\hline \#3 & $\begin{array}{l}\text { Search "Tuberculosis"[Mesh] or ("Tuberculosis, Multidrug-Resistant"[Mesh]) OR "Extensively Drug- } \\
\text { Resistant Tuberculosis"[Mesh] }\end{array}$ \\
\hline \#4 & Search ((\#3) OR \#2) OR \#1 \\
\hline \#5 & Search Xpert* or GeneXpert or Ultra or cepheid Field: Title/Abstract \\
\hline \#6 & Search "near* patient*" or near-patient Field: Title/Abstract \\
\hline$\# 7$ & Search (\#6) OR \#5 \\
\hline \#8 & Search "active case" Field: Title/Abstract \\
\hline \#9 & Search "case finding" Field: Title/Abstract \\
\hline$\# 10$ & Search prevalence Field: Title/Abstract \\
\hline$\# 11$ & Search Asymptomatic Field: Title/Abstract \\
\hline \#12 & Search comorbidity or co-morbidity Field: Title/Abstract \\
\hline \#13 & Search screening Field: Title/Abstract \\
\hline \#14 & Search Detect ${ }^{\star}$ or missed or undetect ${ }^{\star}$ or undiagnosed Field: Title/Abstract \\
\hline \#15 & Search $((((((\# 14)$ OR \#13) OR \#12) OR \#11) OR \#10) OR \#9) OR \#8 \\
\hline \#16 & Search (\#4) AND \#7 AND \#15 \\
\hline
\end{tabular}

\section{Database: Embase 1947-present, updated daily}

Search Strategy:

1 (tuberculosis or TB).mp.

2 Tuberculosis, Multidrug-Resistant/ or Extensively Drug-Resistant Tuberculosis/ or Tuberculosis/ or tuberculosis.mp. or Mycobacterium tuberculosis/

3 (MDR-TB or XDR-TB).mp.

41 or 2 or 3

5 Xpert* MTB RIF.ti. or Xpert* MTB RIF.ab.

6 (Xpert* or GeneXpert or cepheid).mp.

7 (near ${ }^{\star}$ patient or near-patient).ti. or (near ${ }^{\star}$ patient or near-patient).ab.

85 or 6 or 7

94 and 8

10 detection.mp.

Xpert MTB/RIF and Xpert Ultra assays for screening for pulmonary tuberculosis and rifampicin resistance in adults, irrespective of signs or symptoms (Review)

Copyright $\odot 2021$ The Authors. Cochrane Database of Systematic Reviews published by John Wiley \& Sons, Ltd. on behalf of The Cochrane Collaboration. 
11 diagnostic error/ or missed.mp.

12 (undetected or undiagnosed).mp.

13 asymptomatic.mp.

14 comorbidity.mp. or comorbidity/

15 prevalence/

16 active case finding.mp. or case finding/

1710 or 11 or 12 or 13 or 14 or 15 or 16

189 and 17

\section{Web of Science}

\begin{tabular}{lll}
\hline$\#$ & 3 & $\begin{array}{l}\text { \#2 AND \#1 } \\
\text { Indexes=SCI-EXPANDED, SSCI, CPCI-S, CPCI-SSH Timespan=All years }\end{array}$ \\
\hline$\#$ & 2 & $\begin{array}{l}\text { TOPIC: (asymptomatic or undetected or undiagnosed) OR TOPIC: ("case find- } \\
\text { ing" or prevalence or comorbidity) }\end{array}$ \\
\hline Indexes=SCI-EXPANDED, SSCI, CPCI-S, CPCI-SSH Timespan=All years \\
\hline
\end{tabular}

\section{Scopus}

( TITLE-ABS-KEY ( tuberculosis OR tb OR mycobacterium ) AND TITLE-ABS-KEY ( ( xpert* OR genexpert OR cepheid ) ) AND TITLE-ABS-KEY ( asymptomatic OR undetected OR undiagnosed OR "case finding" OR prevalence OR comorbidity ))

\section{LILACS}

(tuberculosis OR TB OR mycobacterium) (Words) AND (xpert\$ OR Genexpert OR Cepheid) (Words)

\section{Cochrane Infectious Diseases Specialized Register}

(tuberculosis or TB) and (xpert* or Genexpert or Cepheid)

\section{Clinicaltrials.gov, WHO ICTRP, ISRCTN:}

Tuberculosis and Genexpert

Tuberculosis and Xpert

\section{ProQuest Dissertations \& Theses}

tuberculosis and (Xpert or genexpert)

\section{Appendix 2. QUADAS-2}

In QUADAS-2, we assessed methodological quality separately for each of the objectives, Xpert for pulmonary tuberculosis detection and Xpert for rifampicin resistance detection.

\section{Domain 1: patient selection}

\section{Xpert MTB/RIF and Xpert Ultra for detection of pulmonary tuberculosis}

Risk of bias: could the selection of patients have introduced bias?

Signalling question 1: was a consecutive or random sample of patients enrolled? We answered 'yes' if the study enrolled a consecutive or random sample of eligible patients; 'no' if the study selected patients by convenience; and 'unclear' if the study did not report the manner of patient selection or we could not tell. 
Signalling question 2: did the study avoid inappropriate exclusions? We answered 'yes' if the study included all individuals in the general population or the high-risk group considered for tuberculosis screening. We answered 'no' if the study primarily or exclusively included individuals with a history of tuberculosis; individuals who had undergone previous treatment (retreatment patients); or those with signs and symptoms of tuberculosis. We answered 'unclear' if we could not tell.

Applicability: are there concerns that the included patients and setting do not match the review question?

We were interested in how Xpert MTB/RIF or Xpert Ultra performed in patients who were evaluated as they would be in the settings of intended use. We answered 'low concern' if the study population resembled a population that was selected for tuberculosis screening in community settings or primary care centres. We answered 'high concern' if the study population does not resemble a population that was selected for tuberculosis screening in a community setting. We answered 'unclear concern' if there was insufficient information to make a decision.

\section{Xpert MTB/RIF and Xpert Ultra for detection of rifampicin resistance}

Domain 1: patient selection is the same as for MTB/RIF or Xpert Ultra for detection of pulmonary tuberculosis.

\section{Domain 2: index test}

\section{Xpert MTB/RIF and Xpert Ultra for detection of pulmonary tuberculosis}

Risk of bias: could the conduct or interpretation of the index test have introduced bias?

Signalling question 1: were the index test results interpreted without knowledge of the results of the reference standard? We answered this question 'yes' for all studies because Xpert test results were automatically generated and the user was provided with printable test results. Thus, there is no room for subjective interpretation of test results.

Signalling question 2: if a threshold was used, was it prespecified? The threshold was prespecified in all versions of Xpert. We answered this question 'yes' for all studies.

For risk of bias, we judge 'low concern' for all studies.

Applicability: are there concerns that the index test, its conduct, or its interpretation differ from the review question? Variations in test technology, execution, or interpretation may affect estimates of the diagnostic accuracy of a test. All steps in the Xpert MTB/RIF and Xpert Ultra assays are completely automated and self-contained following sample loading. We answered 'low concern' if the index test was performed as recommended by the manufacturer, which we had anticipated would be true for most studies. We answered 'unclear concern' if the ratio of the Xpert MTB/RIF or Xpert Ultra sample reagent: specimen volume was not 2:1 for a raw specimen or 3:1 for a sediment, as recommended by the manufacturer.

\section{Xpert MTB/RIF and Xpert Ultra for detection of rifampicin resistance}

Domain 2: index test is the same as for MTB/RIF or Xpert Ultra for detection of pulmonary tuberculosis.

\section{Domain 3: reference standard}

\section{Xpert MTB/RIF and Xpert Ultra for detection of pulmonary tuberculosis}

Risk of bias: could the reference standard, its conduct, or its interpretation have introduced bias?

Signalling question 1: is the reference standard likely to correctly classify the target condition?

We answered 'yes' for all studies, since culture as a reference standard was a criterion for inclusion in the review.

Signalling question 2: were the reference standard results interpreted without knowledge of the results of the index test?

We answered 'yes' if the reference test provided an automated result (for example, MGIT 960), blinding was explicitly stated, or it was clear that the reference standard was performed at a separate laboratory and/or performed by different people. We answered 'no' if the study stated that the reference standard result was interpreted with knowledge of the Xpert MTB/RIF or Xpert Ultra test result. We answered 'unclear' if we could not tell.

Applicability: are there concerns that the target condition as defined by the reference standard does not match the question? We answered 'high concern' if included studies did not speciate mycobacteria isolated in culture; 'low concern' if speciation was performed; and 'unclear concern' if we could not tell.

\section{Xpert MTB/RIF and Xpert Ultra for detection of rifampicin resistance}

Risk of bias: could the selection of patients have introduced bias?

Signalling question 1: is the reference standard likely to correctly classify the target condition?

Xpert MTB/RIF and Xpert Ultra assays for screening for pulmonary tuberculosis and rifampicin resistance in adults, irrespective of signs

Copyright (c) 2021 The Authors. Cochrane Database of Systematic Reviews published by John Wiley \& Sons, Ltd. on behalf of The Cochrane

Collaboration. 
We answered 'yes' if either culture-based drug susceptibility testing (DST) or line probe assay (such as MTBDRplus) was used. These are the criteria for inclusion for this objective of the review.

Signalling question 2: were the reference standard results interpreted without knowledge of the results of the index test?

We answered 'yes' if the reference test provided an automated result (for example, MGIT 960), blinding was explicitly stated, or it was clear that the reference standard was performed at a separate laboratory and/or performed by different people. We answered 'no' if the study stated that the reference standard result was interpreted with knowledge of the Xpert MTB/RIF or Xpert Ultra test result. We will answer 'unclear' if we could not tell.

Applicability: are there concerns that the target condition as defined by the reference standard does not match the question? We judged applicability to be of 'low concern' for those studies evaluating Xpert MTB/RIF or Xpert Ultra for rifampicin resistance because these specimens had already been identified as Mycobacterium tuberculosis positive.

\section{Domain 4: flow and timing}

\section{Xpert MTB/RIF and Xpert Ultra for detection of pulmonary tuberculosis detection}

Risk of bias: could the patient flow have introduced bias?

Signalling question 1: was there an appropriate interval between the index test and reference standard? In most included studies, we expected that specimens for Xpert MTB/RIF or Xpert Ultra and culture would be obtained at the same time, when patients were screened. However, even if there were a delay of several days between index test and reference standard, tuberculosis is a chronic disease and we considered misclassification of disease status to be unlikely, as long as treatment was not initiated in the interim. We answered 'yes' if the index test and reference standard were performed at the same time or if the time interval was less than or equal to seven days, 'no' if the time interval was greater than seven days, and 'unclear' if we could not tell.

Signalling question 2: did all patients receive the same reference standard? We answered this question 'yes' for all studies as an acceptable reference standard (either solid or liquid culture) was specified as a criterion for inclusion in the review. However, we acknowledge that it is possible that some specimens could undergo solid culture and others liquid culture. This could potentially result in variations in accuracy, but we thought the variation would be minimal.

Signalling question 3: were all patients included in the analysis? We determined the answer to this question by comparing the number of patients enrolled with the number of patients included in the $2 \times 2$ tables. We answered 'yes' if the numbers matched and 'no' if there were patients enrolled in the study that were not included in the analysis. We answered 'unclear' if we could not tell.

\section{Xpert MTB/RIF and Xpert Ultra for detection of rifampicin resistance}

Domain 4: flow and timing is the same as for Xpert MTB/RIF or Xpert Ultra for detection of pulmonary tuberculosis.

Judgements for 'Risk of bias' assessments for a given domain

- If we answered all signalling questions for a domain 'yes', then we judged risk of bias as 'low'.

- If we answered all or most signalling questions for a domain 'no', then we judged risk of bias as 'high'.

- If we answered only one signalling question for a domain 'no', we discussed further the risk of bias judgement.

- If we answered all or most signalling questions for a domain 'unclear', then we judged risk of bias as 'unclear'.

- If we answered only one signalling question for a domain 'unclear', we discussed further the risk of bias judgement for the domain.

\section{HISTORY}

Protocol first published: Issue 7, 2020

Review first published: Issue 3, 2021

\section{CONTRIBUTIONS OF AUTHORS}

AES, JMR, MK, KRS, and DJH drafted the manuscript. ND and IS wrote the statistical analysis section. All review authors read and approved the final manuscript draft.

\section{DECLARATIONS OF INTEREST}

AES received funding from USAID, administered by the World Health Organization Global TB Programme, Switzerland. She has received salary compensation from the University of Washington, where she is an Acting Assistant Professor in Global Health and Medicine/ Infectious Diseases. A portion of her salary derives from NIH grants and from grants from the Bill \& Melinda Gates Foundation.

Xpert MTB/RIF and Xpert Ultra assays for screening for pulmonary tuberculosis and rifampicin resistance in adults, irrespective of signs 
JMR received funding from USAID, administered by the World Health Organization Global TB Programme, Switzerland. JMR has grants/ grants pending to her host institution from US National Institutes of Health, KNCV TB Foundation, and The Global Fund to Fight AIDS, TB, and Malaria, The Firland Foundation.

MY has no known conflicts of interest to declare.

IS has no known conflicts of interest to declare.

MK has received funding from USAID, administered by the World Health Organization Global TB Programme, Switzerland for related systematic reviews.

ND has no known conflicts of interest to declare.

KRS has received financial support from Cochrane Infectious Diseases, UK, McGill University, Canada, and USAID, USA, administered by the World Health Organization (WHO) Global TB Programme, Switzerland, for the preparation of systematic reviews and educational materials, consultancy fees from Foundation for Innovative New Diagnostics (FIND), Switzerland (for the preparation of systematic reviews and GRADE tables), honoraria, and travel support to attend WHO guideline meetings.

DJH has received funding from USAID, administered by the World Health Organization Global TB Programme, Switzerland for related systematic reviews.

\section{SOURCES OF SUPPORT}

\section{Internal sources}

- Liverpool School of Tropical Medicine, UK

\section{External sources}

- Foreign, Commonwealth and Development Office (FCDO), UK

Project number 300342-104

- United States Agency for International Development (USAID), USA

Development of this project was in part made possible with financial support from USAID administered by the World Health Organization Global TB Programme

\section{DIFFERENCES BETWEEN PROTOCOLANDREVIEW}

Objectives: we intended to assess Xpert MTB/RIF and Xpert Ultra as screening tests for pulmonary tuberculosis in the general population, irrespective of signs and symptoms. However, we did not identify any studies conducted in the general population.

Types of studies: We included abstracts with sufficient data to populate $2 \times 2$ contingency tables.

Statistical analysis and data synthesis: we performed a post-hoc analysis by combining several high-risk groups into a single pooled analysis of sensitivity and specificity in adults at high risk for tuberculosis. These high-risk groups included household contacts of people with tuberculosis, people residing in prisons, and miners. We had planned to compare the accuracy of Xpert MTB/RIF and Xpert Ultra by first including all studies with relevant data, i.e. both indirect and direct comparisons, and then by restricting the analyses to studies that made comparisons between Xpert MTB/RIF and Xpert Ultra in the same participants, i.e. direct comparisons. However, there were insufficient data to perform these comparisons. We stated in the protocol that we would provide predictive sensitivity and specificity. We did not do this. However, instead, we estimated positive and negative predictive values at pre-specified pre-test probabilities recommended by the WHO $(0.5 \%$ and $5 \%)$ as we considered these values more useful for clinicians.

Subgroup analyses: we intended to perform subgroup analyses among studies conducted in high versus not high tuberculosis burden countries, and similarly for high TB/HIV burden and high MDR-TB burden versus not high burden countries. However, most studies were conducted in high burden countries, therefore we did not perform these subgroup analyses.

Sensitivity analyses: we had planned to perform sensitivity analyses limiting the analyses to studies that accounted for all participants in the analysis, studies that used liquid culture as the reference standard, and studies where a consecutive or random sample of participants were enrolled. However, all studies met these criteria, therefore we were unable to perform these sensitivity analyses.

Uninterpretable results: regarding uninterpretable results, in the protocol we wrote we would use a Bayesian hierarchical model for a single proportion to estimate the pooled proportion of uninterpretable index test results. However, most studies in this review did not report uninterpretable results, so we did not model them separately.

Xpert MTB/RIF and Xpert Ultra assays for screening for pulmonary tuberculosis and rifampicin resistance in adults, irrespective of signs 
Non-stigmatising language: whenever possible, we re-categorized high-risk groups using non-stigmatising language. For example, we changed the category "homeless people" to "people experiencing homelessness."

\section{NDEX TERMS}

\section{Medical Subject Headings (MeSH)}

Antibiotics, Antitubercular [*pharmacology]; Bacteriological Techniques [methods]; Bayes Theorem; Bias; Cohort Studies; CrossSectional Studies; *Drug Resistance, Bacterial; False Negative Reactions; False Positive Reactions; HIV Infections [complications]; Mycobacterium tuberculosis [ ${ }^{*}$ drug effects] [isolation \& purification]; Polymerase Chain Reaction [ ${ }^{*}$ methods]; Rifampin [ ${ }^{\star}$ pharmacology]; Sensitivity and Specificity; Sputum [microbiology]; Tuberculosis, Pulmonary [complications] [ ${ }^{\star}$ diagnosis] [drug therapy]

\section{MeSH check words}

Adult; Humans 